\title{
Wild edible plants in Ethiopia: a review on their potential to combat food insecurity
}

\author{
Ermias Lulekal (1), Zemede Asfaw (2), Ensermu Kelbessa (2), \\ Patrick Van Damme $(1,3)$ \\ (I) Department of Plant Production, Ghent University, Belgium \\ (2) Department of Biology, Addis Ababa University, Ethiopia \\ (3) ICRAF, Nairobi, Kenya
}

\begin{abstract}
This work reviews literature on ethnobotanical knowledge of wild edible plants and their potential role in combating food insecurity in Ethiopia. Information on a total of 413 wild edible plants belonging to 224 genera and 77 families was compiled in this review. Shrubs represented $31 \%$ of species followed by trees $(30 \%)$, herbs $(29 \%$ ) and climbers ( $9 \%$ ). Families Fabaceae ( 35 species), Tiliaceae (20) and Capparidaceae (19) were found to be represented by the highest number of edible species. About $56 \%$ (233) of species have edibility reports from more than one community in Ethiopia. Fruits were reported as the commonly utilized edible part in $5 \mathrm{r} \%$ of species. It was found that studies on wild edible plants of Ethiopia cover only about $5 \%$ of the country's districts which indicates the need for more ethnobotanical research addressing all districts. Although there have been some attempts to conduct nutritional analyses of wild edible plants, available results were found to be insignificant when compared to the wild edible plant wealth of the country. Results also show that wild edible plants of Ethiopia are used as supplementary, seasonal or survival food sources in many cultural groups, and hence play a role in combating food insecurity. The presence of anthropogenic and environmental factors affecting the wild plant wealth of the country calls for immediate action so as to effectively document, produce a development plan and utilize the plants.
\end{abstract}

Key words: ethnobotany, cultural diversity, food insecurity, indigenous knowledge, nutritional analysis

\section{Introduction}

Ethnobotanical investigations into wild edible plants

Wild edible plants (WEPs) refer to species that are neither cultivated nor domesticated, but are available from their wild natural habitat and used as sources of food (Beluhan and Ranogajec, 2010). Despite the primary reliance of most agricultural societies on staple crop plants, the tradition of eating WEP products continues in the present day. In addition to their role in closing food gaps during periods of drought or scarcity, WEPs play an important role in maintaining livelihood security for many people in developing countries (Afolayan and Jimoh, 2009). 
WEPs have been a focus of research for many ethnobotanists in recent decades. Currently, there is renewed global interest in documenting ethnobotanical information on neglected wild edible food sources (Bharucha and Pretty, 2010). Since traditional knowledge on WEPs is being eroded through acculturation and the loss of plant biodiversity along with indigenous people and their cultural background, promoting research on wild food plants is crucial in order to safeguard this information for future societies (Asfaw, 2009).

A major objective of ethnobotanical investigation into wild food plants is the documentation of indigenous knowledge associated with these plants. Comparative studies on WEPs in different cultures or ethnic groups of a country or among different countries, may contribute to the identification of the most widely used species for further nutritional analysis (Termote et al., 2009; De Caluwé, 2010a and 2010b). Nutritional analysis results provide clues to aid the promotion of those species that have the best nutritional values which helps to to ensure dietetic diversity and combat food insecurity (Tardio et al., 2006).

A considerable amount of research has been conducted worldwide on WEP ethnobotany with an emphasis on field surveys and documentation, to cite but a few: Asfaw and Tadesse (2001); Pieroni et al. (2002); Ertug (2004); Reyes-Garcia et al.(2005); Balemie and Kibebew (2006); Tardio et al. (2006); Arenas and Scarpa (2007); Rashid et al. (2008); Asfaw (2009); Giday et al. (2009); and Teklehaymanot and Giday (2010). Moreover, research on nutritional value and health benefits of WEPs has been reported from Grivetti and Ogle (2000); Ohiokpehal (2003); Heinrich et al. (2005); Balemie and Kibebew (2006); Termote et al. (2009, 2010 and 2011), De Caluwé (2010a and 2010b); Beluhan and Ranogajec (2010) and Feyssa et al. (2011). Regardless of the numerous efforts to document WEPs and associated indigenous knowledge, underestimation of the value of these WEPs can lead to the neglect of ecosystems that nurture them and the indigenous knowledge systems that are related to them (Pilgrim et al., 2008). Hence, we found it worthwhile to go through ethnobotanical information on WEPs of Ethiopia and compile existing information as a basis lead for further investigations into these plants.

\section{Potential contribution to food security}

When compared to domesticated plant food sources, wild plant foods tend to be overlooked. However there is substantial evidence that indicates the importance of wild edibles in terms of the global food basket. Since WEPs are freely accessible within natural habitats, indigenous people have knowledge of how to gather and prepare the foods (Somnasang and Moreno-Black, 2000). According to Abermound (2009), about one billion people in the world use wild foods (mostly from plants) on a daily basis. Moreover, over 300 million people obtain a substantial part of their livelihood in the form of NonTimber Forest Products (NTFPs) from wild forests (Belcher et al., 2005).

According to Jaenicke and Hoschle-Zeledon (2006), over 50 percent of the world's daily requirement of proteins and calories is obtained from only three crops: wheat, maize and rice. The dependence on a few domesticated species limits dietetic diversity and leads 
to over dependence on limited resources. By contrast, ethnobotanical investigations on WEPs suggest that more than 7,000 species have been used for food in human history (Grivetti and Ogle, 2000). In countries such as China, India, Thailand and Bangladesh hundreds of WEPs are still consumed along with domesticated species (Mazhar et al., 2007). The document of Rathore (2009) shows the presence of 600 WEP species in India. Moreover, Boa (2004) documented the presence of over Iooo species of wild edible fungi (which do not belong to the plant kingdom but are closely related to it) worldwide. These figures show the intimate link between WEPs and the diets of many people, hence wild edibles can be considered to be useful resources in the efforts to achieve food security. Further to their roles in food security, many WEPs such as Adansonia digitata L. (Malvaceae), Moringa stenopetala (Bak. f.) Cufod. (Moringaceae), Syzygium guineense (Willd.) DC. (Myrtaceae) and Ximenia americana L. (Olacaceae) are acknowledged for their medicinal, cultural, forage and economic values (Johns et al., I996; Ogle et al., 2003; Reyes-Garcia et al., 2005; Shrestha and Dhillon, 2006).

\section{Nutritional value of WEPs}

Leaves, stems, fruits, flowers, tubers, barks, seeds, roots, and so on, of lots of WEPs are still consumed for their dietary value in many communities around the globe. Some of these WEPs are used as primary food sources while others are used as secondary condiments in dishes prepared from domesticated cultivars (Lockett and Grivetti, 2000). These plants play an important role as a source of energy and micronutrients (Afolayan and Jimoh, 2009; De Caluwé, 20roa and 20rob). Currently, preliminary research results on dietary analysis of many WEPs provide promising information (Table 1. .) 


\begin{tabular}{|c|c|c|c|c|c|}
\hline No & WEP species & Family & Nutritional value & Where? & Source \\
\hline I & Abrus precatorius $\mathrm{L}$. & Fabaceae & $\begin{array}{l}\text { Protein, } \mathrm{Ca}, \mathrm{Fe}, \mathrm{K}, \\
\mathrm{Na}, \mathrm{Mg}, \mathrm{Mn} \text { and } \mathrm{Zn}\end{array}$ & \multirow[t]{3}{*}{ Cameroun } & \multirow[t]{3}{*}{$\begin{array}{l}\text { Glew et } \\
\text { al., 20I0 }\end{array}$} \\
\hline 2 & $\begin{array}{l}\text { Burnatia enneandra } \\
\text { Micheli }\end{array}$ & Alismataceae & $\begin{array}{l}\text { Protein, } \mathrm{Ca}, \mathrm{Fe}, \mathrm{K} \text {, } \\
\mathrm{Na}, \mathrm{Mg}, \mathrm{Mn} \text { and } \mathrm{Zn}\end{array}$ & & \\
\hline 3 & Cadaba farinosa & Capparidaceae & $\begin{array}{l}\text { Protein, fat, } \mathrm{Ca}, \mathrm{Fe} \text {, } \\
\mathrm{K}, \mathrm{Na}, \mathrm{Mg}, \mathrm{Mn} \text { and } \\
\mathrm{Zn}\end{array}$ & & \\
\hline 4 & Agave salmiana Otto & Agavaceae & \multirow[t]{6}{*}{ Protein } & \multirow[t]{6}{*}{ Mexico } & \multirow{6}{*}{$\begin{array}{l}\text { Lopez- } \\
\text { Garcia \& } \\
\text { Basurto- } \\
\text { Pena, } \\
2007\end{array}$} \\
\hline 5 & Aloe vera $\mathrm{L}$. & Aloaceae & & & \\
\hline 6 & $\begin{array}{l}\text { Arbutus xalapensis } \\
\text { Kunth }\end{array}$ & Ericaceae & & & \\
\hline 7 & $\begin{array}{l}\text { Erythrina americana } \\
\text { Mill. }\end{array}$ & Fabaceae & & & \\
\hline 8 & $\begin{array}{l}\text { Euphorbia radians } \\
\text { Benth. }\end{array}$ & Euphorbiaceae & & & \\
\hline 9 & Yucca filifera Chabaud & Agavaceae & & & \\
\hline 10 & Ximenia americana L. & Olacaceae & $\mathrm{Ca}, \mathrm{P}, \mathrm{Mg}$ & \multirow[t]{7}{*}{ Niger } & \multirow{7}{*}{$\begin{array}{l}\text { Freiberger } \\
\text { et al., } 1998\end{array}$} \\
\hline II & Amaranthus viridis L. & Amaranthaceae & Protein, $\mathrm{Ca}, \mathrm{Fe}, \mathrm{P}$ & & \\
\hline 12 & Corchorus tridens L. & Tiliaceae & Protein, fat, $\mathrm{P}, \mathrm{Cu}$ & & \\
\hline 13 & Hibiscus sabdariffa L. & Malvaceae & Protein, fat, $\mathrm{P}$ & & \\
\hline I4 & $\begin{array}{l}\text { Maerua Crassifolia } \\
\text { Forssk }\end{array}$ & Capparidaceae & Fat, Ca, P & & \\
\hline 15 & Moringa oleifera Lam. & Moringaceae & Protein, fat, $\mathrm{P}$ & & \\
\hline I6 & $\begin{array}{l}\text { Leptadenia hastata } \\
\text { Decne }\end{array}$ & Asclepiadaceae & Protein, fat, $\mathrm{Ca}, \mathrm{P}$ & & \\
\hline 17 & $\begin{array}{l}\text { Borassus aethiopum } \\
\text { Mart. }\end{array}$ & Arecaceae & Protein, zn & \multirow[t]{2}{*}{$\begin{array}{l}\text { Sahel } \\
\text { region }\end{array}$} & \multirow[t]{2}{*}{$\begin{array}{l}\text { Glew et } \\
\text { al., } 2005\end{array}$} \\
\hline I8 & Tamarindus indica L. & Fabaceae & $\begin{array}{l}\text { Carbohydrate, pro- } \\
\text { tein and fat }\end{array}$ & & \\
\hline I9 & Portulaca oleracea L. & Portulacaceae & Protein and fat & \multirow[t]{2}{*}{ India, Iran } & \multirow{2}{*}{$\begin{array}{l}\text { Aberou- } \\
\text { mand \& } \\
\text { Deokule, } \\
2009\end{array}$} \\
\hline 20 & Asparagus officinalis L. & Asparagaceae & Protein and fat & & \\
\hline
\end{tabular}

Table 1. Nutritional analysis reports on WEPs in some countries

In addition, Becker $(1983)$ reported the presence of vitamins $\mathrm{A}, \mathrm{B2}$, and $\mathrm{C}$ in WEPs of Senegal. Research on six WEPs from Spain also confirmed the occurence of lipids, fatty acids and carotenes in the leaves of these species (Guill-Guerrero and Rodriguez-Garcia, 
1999). Protein content in a proportion that is comparable to the amount in domesticated plants was also reported from a nutritional study of WEPs in South Africa (Afolayan and Jimoh, 2009). A study on the dietary value of eight wild edibles in Iran and India also showed the presence of sodium, calcium, potassium, iron, zinc, protein, and fat in a ratio comparable to that found in cultivated plants (Aberoumand, 2009). Many wild leafy vegetables of Poland are also mentioned for their rich source of vitamin $C$, natural antioxidants, carotenoids and folic acid (Luczaj, 20ro). Generally, the information available from the nutritional analysis of WEPs shows their potential contribution to dietetic diversity and food security.

\section{WEPs in Ethiopia}

Ethiopia is a country with varied a topography and a wide spectrum of habitats presenting a large number of endemic plants and animals. The country has about 6000 higher plant species of which about 10\% are endemic (Hedberg et al., 2009). Ethiopia also harbours two of the 34 global biodiversity hotspots (CI, 2004) and is recognized as a Vavilov centre of origin and diversification for many food plants and their wild relatives (Edwards, 199I). Forests, grasslands, riverine environments and wetlands are home to numerous WEPs in the country (Asfaw, 2009). Local communities in Ethiopia are also endowed with diverse indigenous knowledge, related to the rich biodiversity of the country.

In most parts of Ethiopia, wild edibles form integral parts of the feeding habits of many communities (Balemie and Kibebew, 2006). However, consumption of wild edibles is more common in food insecure areas than in other areas in the country (Teklehaymanot and Giday, 2010). For example, the Konso people in southern Ethiopia managed to endure three severe drought seasons of crop failure between 1996 and 1999 by consuming WEPs available in the region (Guinand and Lemessa, 200I). Despite the wide availability and utilization of WEPs in Ethiopia, ethnobotanical information on cultural, socio-economic and nutritional values of Ethiopian plants is limited. Hence, there is still a need for documentation, nutritional analysis and domestication of WEPs to assist in the nationwide effort to combat food insecurity and ensure dietetic diversity.

\section{Objectives and methodology}

The purpose of the present work is to review existing literature on ethnobotanical knowledge of WEPs and their role in combating food insecurity in Ethiopia. Although there are 85 cultural groups and a great number of WEPs in Ethiopia, past research has only addressed a statistically insignificant proportion of them. This work intends to document the status of available ethnobotanical information on WEPs in the country so as to identify existing gaps in research and information on Ethiopian WEPs. Hence as offered by Web of science (WOS), the authors have included all information on WEPs of Ethiopia from published documents including journal articles, books, reports and proceedings. The literature search also addressed online publications on WEPs from other countries in order to point out useful research practices that could be used for future ethnobotanical research on WEPs of Ethiopia. Databases comprising information on WEPs were 
browsed using the following main search terms: 'wild edible plants', 'wild edible plants of Ethiopia', 'ethnobotanical study of wild edible plants' and 'food security in Ethiopia'.

Data on Ethiopian WEPs with their scientific and local names, growth form, parts used and specific cultural groups consuming the plants was gathered and compiled after assessing all available Ethiopian ethnobotanical documents. Data was then entered in an Excel spreadsheet and analysed using descriptive statistics to identify the number and percentage of species, genera and families of WEPs, their growth forms and percentage of commonly utilized plant parts. The output of this review can serve as a basis for future ethnobotanical studies on WEPs of Ethiopia. Readers are referred to original articles on Ethiopian WEPs for detailed analytical methods and interpretation of results; all resources used for this review are duly cited.

\section{Results and discussion}

Traditional knowledge on wild edible plants of Ethiopia

In total, ethnobotanical information on 413 WEPs belonging to 224 genera and 77 families was compiled (Appendix 1 ). About $17 \%$ of families were found to be represented by more than Io edible species, $18 \%$ had 5 to Io species, $36 \% 2$ to 4 , whereas the remaining $29 \%$ of families were represented by single species only. Families Fabaceae ( 35 species), Tiliaceae (20) and Capparidaceae (19) were found to be represented by the highest number of edible species (Fig. I).

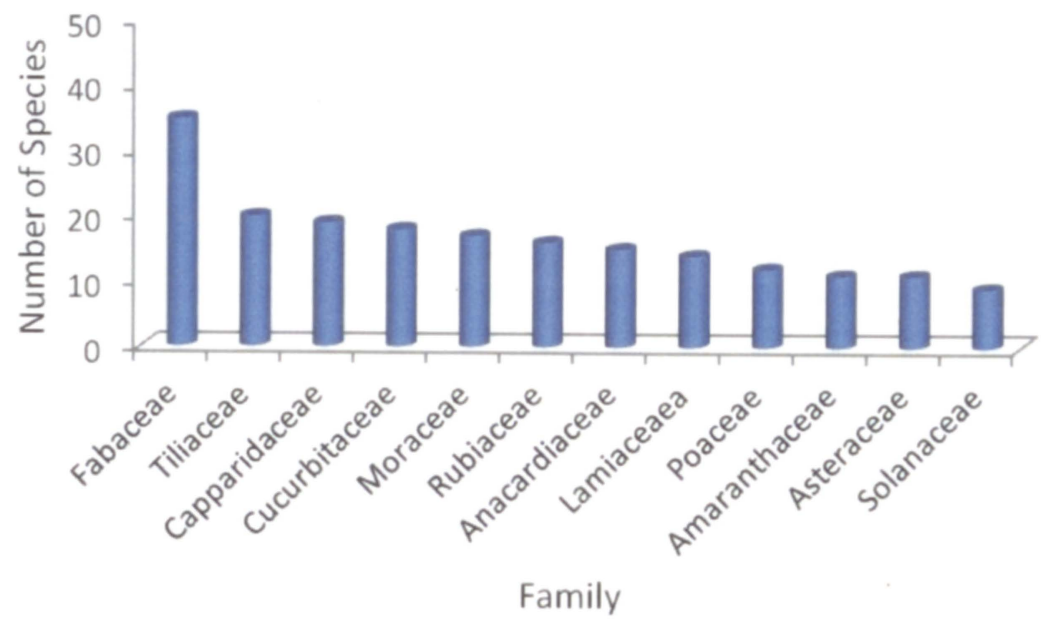

Figure 1. Families represented by highest number of WEP species in Ethiopia

Results show that the country is rich in WEP diversity. The wide utilization of wild edible species of the family Fabaceae could be attributed to the highest number of species in Ethiopian Flora residing in it besides their wider distribution. 
Growth form analysis of Ethiopian WEPs indicates that shrubs (I 29 species, 32\%,) represented the dominant growth form, followed by trees ( 127 species, 30\%), herbs (I2I species, $29 \%$ ) and climbers (36 species, $9 \%$ ), respectively. Fruits were the most commonly reported edible parts of about $51 \%$ (210 species) of plants reported for food, followed by leaves ( 97 species, $23 \%$ ) and seeds ( 43 species, I0\%) (Fig. 2). Moreover, other parts or products such as gum, nectar, bark, inflorescence, tubers or a combination of two or more of these parts or products were also reported for edibility in different communities. This indicates that the different cultural groups in Ethiopia make use of diverse WEP parts as food sources.

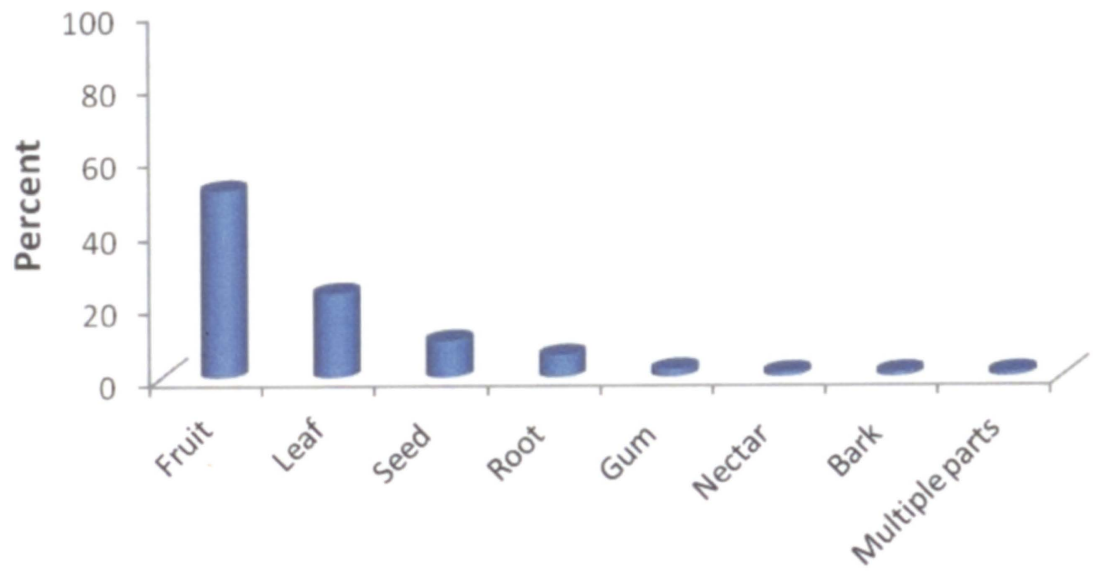

Edible parts

Figure 2. The most commonly reported edible parts of Ethiopian WEPS

A total of 233 species ( $56 \%$ ) were also reported as edible from more than one community in Ethiopia. This shows that the different cultural groups of the country share relatively similar knowledge on WEPs utilisation. Moreover, it shows that the age-old cultural practice of using WEPs brought people to share the knowledge regarding identification, preparation and consumption of similar WEPs.

Status of research into WEPs of Ethiopia

In this review, it was found that the existing ethnobotanical information on WEPs of Ethiopia is very limited and fragmentary. The areas in Ethiopia for which at least some information has been documented for their WEPs were: southwestern Ethiopia (Abbink, 1993; Guinand and Lemessa, 200I), Alamata, Cheha, Goma, Yilmana Densa districts (Addis et al., 2005), Derashe and Kucha districts (Balemie and Kibebew, 2006), Dera town in Dodotana Sire district (Wondimu et al., 2006), Hamar and Xonso districts (Ad- 
dis, 2009), Addi Arkay, Debark and Dejen districts (Fantahun and Hager, 2010), Kara and Kewego people of South Omo zone in Kuraz and Hamar districts (Teklehaymanot and Giday, 20I0) and Fantalle and Boosat districts (Feyssa et al., 201I)(Fig. 3). Moreover, the ethnobotanical writings by Getahun (1974), Asfaw and Tadesse (200I) and Asfaw (2009) were found to give some general information on WEPs in the country. In addition, a book by Teketay et al. (2010) was found to be the only one that provides information on 378 WEPs of Ethiopia of which only 262 were presented with specific locality information where they are utilised, while the remaining 116 species were found to be listed in appendices with no locality information. Hence, it was found that all available ethnobotanical research outputs on Ethiopian WEPs address only about 5 percent of the 494 Ethiopian districts which is an insignificant share when the immense geographic, ethnic and cultural diversity of the country is taken into account. Moreover, research appears to focus only on commonly known and widely available plants most of which occur in the central and highland regions of the country. 


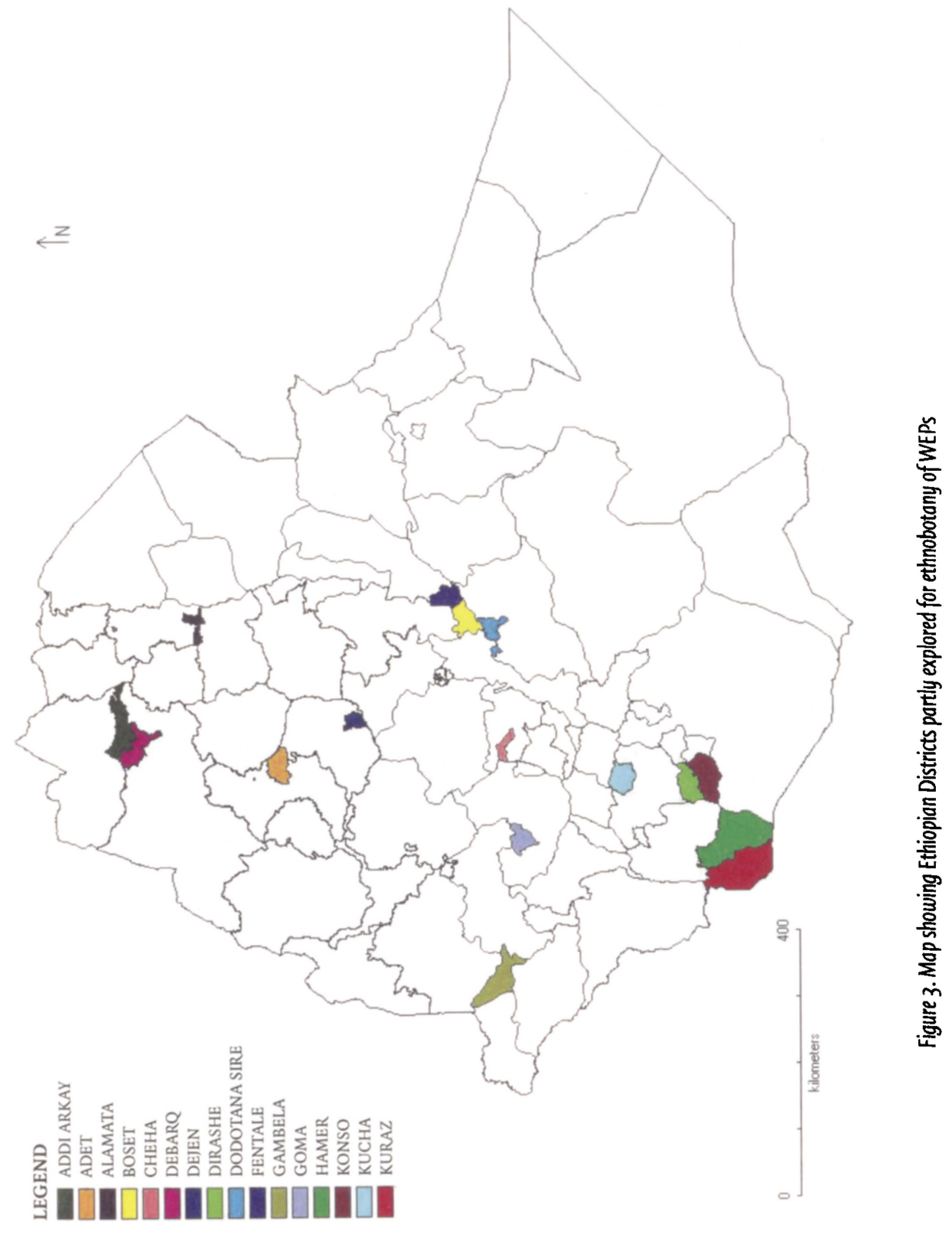


None of the available research outputs addressed the drier lowlands of Ethiopia where many cultural groups live with indigenous knowledge of WEPs that are used during dry spells. Moreover, earlier research outputs on WEPs of Ethiopia were not systematic and mainly only report names of plants. It was observed that current research on Ethiopian WEPs is becoming more systematic focusing on indigenous knowledge of specific communities as noted in the writings of Addis (2009).

Among the list of WEPs, the species most commonly reported for utilization include Urtica simensis Steudel. (endemic species), Cordia africana Lam., Ximenia americana L., Tamarindus indica, L., Syzygium guineense (Willd. ) DC., Dovyalis abyssinica (A. Rich.) Warb, Ficus sur Forssk, Ficus vasta Forssk, Physalis peruviana L., Rosa abyssinica Lindley, Rubus steudnerii Schweinf, Carissa spinarum L., Moringa stenopetala (Bak.f.) Cuf., Opuntia ficus-indica (L.) Miller, and Solanum nigrum L. (Fig. 4) (Appendix I) (Balemie and Kibebew, 2006; Addis, 2009; Teklehaymanot and Giday, 2010; Fantahun and Hager, 2010; Teketay et al., 2010). However, we did not find any nutritional analysis of these commonly reported species.

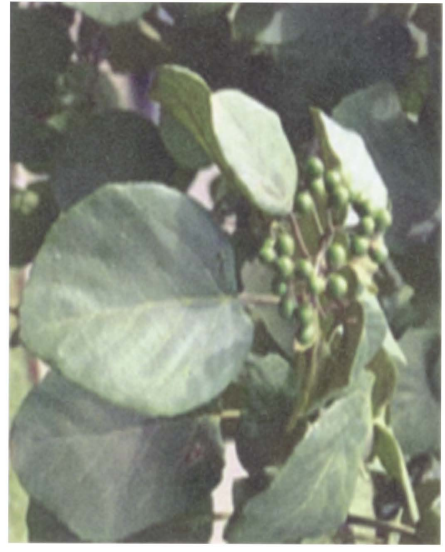

Cordia africana Lam.

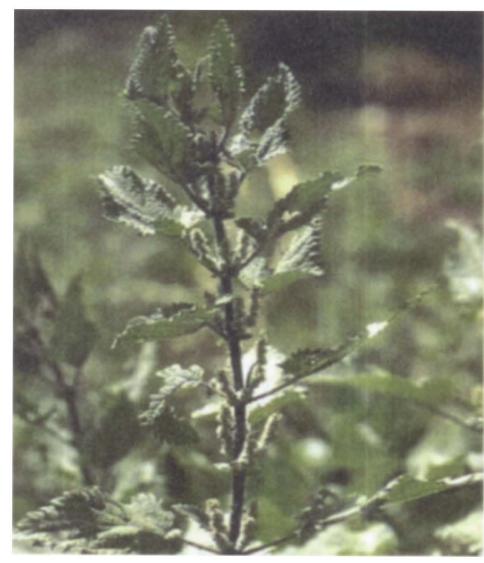

Utrica simensis Steudel 


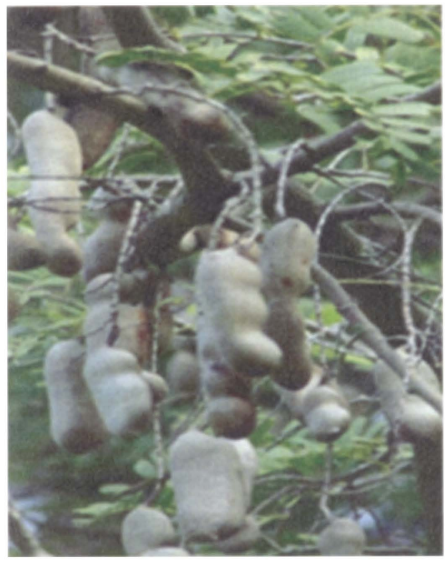

Tamarindus indica $\mathrm{L}$.

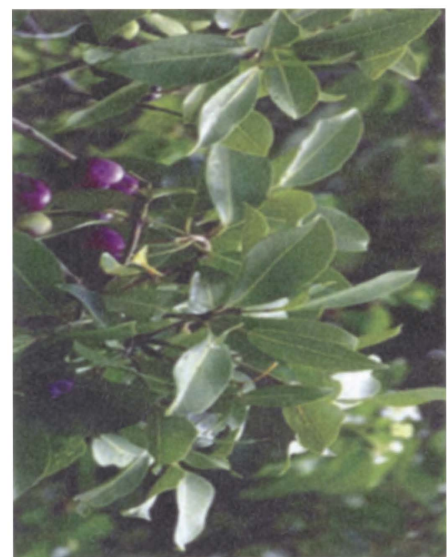

Syzygium guineense (Willd.) DC.

Figure 4. Some of the most commonly reported Ethiopian WEPs. (Photo source: http://database.prota.org)

Knocking at the gate of Ethiopian wild habitats

Many WEPs from other countries were also found reported for their occurrence in Ethiopian wild habitats, but with no ethnobotanical information about their edibility. For instance, wild plant species including Abutilon hirtum (Lam.) Sweet, Asparagus racemosus Willd., Centella asiatica (L.) Urban, Chenopodium album L., Commelina africana L., Pennisetum purpureum Schumach. and Plantago lanceolata L. are documented for edibility in other countries as noted in the writings of Ryan (2000); Ghirardini et al. (2007); Bandyopadhyay and Mukherjee (2008); Misra et al. (2008); Afolayan and Jimoh (2009); Okaraonye and Ikewuchi (2009) and Binu (2010). However, despite their importance no information on the edibility of these species was found in any of the Ethiopian ethnobotanical documents. This shows the need to carry out further comparative ethnobotanical studies on WEPs of Ethiopia and other countries, so that the information could be used as a tool to further research Ethiopian wild habitats to identify unexplored, but reportedly important, WEPs in the country.

\section{Ethiopian wild edibles and their role in combating food insecurity}

According to FAO (2010), more than $35 \%$ of Ethiopian people are food insecure. The country's ever increasing population along with recurrent drought, war and poor agricultural practices with low productivity, have pulled the country into a vicious circle of food insecurity. In addition, over dependence on a limited number of food sources, and poor efforts to diversify dietary sources aggravate the country's food insecurity problem.

Many WEPs in Ethiopia were reported as emergency, supplementary or seasonal food sources to avert food insecurity in households of Ethiopian cultural groups. For example, the invasive Opuntia ficus-indica (L.) Miller (Cactaceae), was found to be widely exploited for its fruit in many parts of northern Ethiopia, playing a significant role in food 
source diversification (Addis, 2009). The fruits of this plant are also sold in many local markets in the Tigray regionof Ethiopia along with other cultivated food sources such as potato, carrot, bean and maize. Amorphophallus gallaensis (Engl.) N. E. Br and Caralluma sprengeri N. E. Br. were also reported for their role in fighting food insecurity during periods of drought and famine in Konso district (Guinand and Lemessa, 2000). This shows the role that WEPs of Ethiopia play, at least at local levels, to combat food insecurity and their potential to address existing food insecurity at national level if properly managed.

WEPs in Ethiopia that are reported to have nutritional and commercial properties that are valued in other countries (for example, Adansonia digitata L., Tamarindus indica L., and Ziziphus mauritiana Lam.) are found to be underutilized in the country. Hence it is important that policy and decision makers consider all available ethnobotanical information on Ethiopian WEPs so as to develop regional and national plans for the conservation, management and sustainable utilization of the country's underused wealth of WEPs .

\section{Conservation of WEPs in Ethiopia}

Despite their importance, WEPs face serious anthropogenic and environmental threats. Many threats are similar to those that affect plant diversity as a whole. The most common threats reported were agricultural expansion, overgrazing/overstocking, deforestation and urbanization (Kelbessa et al., 1992; Addis, 2009; Asfaw, 2009; Teklehaymanot and Giday, 2010). The reported anthropogenic pressures in the country have resulted in a loss of thousands of hectares of forest that harbour useful WEPs. This loss was also reported to limit benefits gained from the plants and indigenous knowledge associated with these plants. The continuity of knowledge on the utilisation of WEPs has also faced problems because of change in the feeding culture of the people (Teklehaymanot and Giday, 2010).

The reviewed research outputs on WEPs of the country indicate the need for conservation as well as documentation (Balemie and Kibebew, 2006; Addis, 2009; Asfaw, 2009; Teklehaymanot and Giday, 2010; Fantahun and Hager, 2010). Conserving Ethiopian WEPs in situ (in their natural habitat as in nature reserves and parks) or ex situ (e.g. in field gene banks, botanic gardens or cold rooms) is mandatory (Teklehaymanot and Giday, 2010). Moreover, effective protection or sustainable management of the 58 National Forest Priority Areas (NFPAs) of the country will play a major role in conserving a great number of WEPs that cannot be economically cultivated, require very specific habitats, and are exceptionally difficult to reproduce in nurseries.

\section{Lessons learnt from other countries}

In the last decade, many countries have given priority to the documenting of WEPs and the associated indigenous knowledge. In countries such as India, Mexico, Bolivia, Spain and Turkey, in-depth ethnobotanical information on WEPs is available (Reyes-Garcia et al., 2005; Tardio et al., 2006; Rashid et al., 2008). By contrast, in this review it was found that research conducted on WEPs of Ethiopia was shallow and addressed only an insignificant portion of the country. Hence, there is a need for extensive research to accumulate WEP knowledge from all 85 cultural groups of the country. 
Existing dietary analyses in Ethiopia were found to be very limited and still to be at a basic stage when compared to studies in other countries. In South Africa, Mexico, Niger and India, for example, in-depth nutritional analyses of many WEPs have been reported (Ogle and Grivetti, 1985; Frieberger et al., 1998; Lopez-Garcia and Basurto-Pena, 2007; Afolayan and Jimoh, 2009; Rathore, 2009; Abdillahi et al., 2010). A report on nutritional value assessment of Ziziphus spina-christi (L) Desf. (Rhamnaceae), Balanites aegyptiaca (L.) Del. (Balanitaceae) and Grewia flavescens A. Juss (Tiliaceae) in Ethiopia shows that these species are rich in carbohydrate, protein, and lipid (Feyssa et al., 20r1). Further research at least on commonly reported Ethiopian WEPs will help to identify more food supplements with rich nutritional values that can help to avert food insecurity. In addition to nutritional analyses, many research reports are also available documenting anti-nutritional and toxicity studies of WEPs from different countries (Guill-Guerrero et al., I997; Vanderjagt et al., 2000; Lopez-Garcia and Basurto-Pena, 2007; Spina et al., 2008), which have not been done for WEPs of Ethiopia except for a limited attempt made by Addis (2009).

Integrating WEPs into agricultural landscapes plays an important role in achieving household food security and in the conservation of plants. The practice of integrating fruit-bearing WEPs into agricultural landscapes has been reported in Uganda, Cameroon and Nigeria (Degrande et al., 2006; Agea et al., 2007). In Ethiopia, Fantahun and Hager (2010), reported the extent of integrating wild fruit-bearing species into agricultural landscapes of the Amhara region, and indicated that about 17 species including Ziziphus spina-christi (L) Desf., Cordia africana Lam.(Boraginaceae), Tamarindus indica L. (Fabaceae) and Rosa abyssinica Lindley (Rosaceae) were found integrated in the agricultural settings in the area. Despite such attempts, the overall practice of integrating WEPs into the agricultural landscapes of Ethiopia is limited when compared to other countries, hence the need to address the gap.

Although some WEPs including Opuntia ficus-indica (L.) Miller, Moringa stenopetala (Bak. f.) Cufod., Sclerocarya birrea (A. Rich) Hochst. and Leptadenia hastata (Pers.) Decne, were reported to be available in rural markets of Ethiopia (Balemie and Kibebew, 2006, Addis, 2009), research on market chain analysis and economic value of these plants has not yet been addressed. A lesson on exploring the economic use of WEPs to supplement household income could be taken from the rational economic assessment of these plants from other countries such as Thailand and India (Delang, 2006, Misra et al., 2008).

In many countries, edible forms of wild mushrooms have been identified, cultivated and incorporated as staple foods (Boa, 2004). Extensive collections and herbarium data have also been documented in different countries (Beluhan and Ranogajec, 2010). In contrast to this, in Ethiopia, a country that possesses numerous species of wild mushroom, (Abate, 1999), there is very little information available on such species.

Tuno (200I) reported on the wild edible mushroom utilization by the Majanjir tribe in Ethiopia, and this was found to be the only attempt to address a specific cultural group. This indicates the need for further assessment and documentation of the wild edible mushroom utilization of all other cultural groups in the country.

Some wild fruits that grow in the forests of Ethiopia were found to have important 
dietetic value in other countries. For example, Ziziphus mauritiana Lam. (Rhamnaceae) yields a sweet edible fruit (Van Damme and Termote, 2008). This plant has now been improved through grafting and genetic technologies to produce bigger, edible fruits with sweeter flavours (Van Damme and Termote, 2008). In addition, Adansonia digitata L. (Malvaceae) is serving as a source of sweet juice and is now widely available from markets in France and Italy (Van Damme and Termote, 2008). Despite the presence of these useful WEPs in Ethiopia, no report was found on their promotion. Hence, to attain dietetic and economic benefit from such useful WEPs in the country extensive promotion activities on target plants are important.

The diverse groups of epiphytes and orchids available in Ethiopia were also found to be unexplored, hence no ethnobotanical data was found on edible forms of these species. A lesson in this respect could be taken from neighbouring Tanzania that possesses documents on 85 edible orchids and epiphytes (Davenport and Ndangalasi, 2003, Challe and Struik, 2008).

\section{Conclusions}

Despite the wide utilization of WEPs in Ethiopia, ethnobotanical information regarding local knowledge of these plants is very limited. Available research information on Ethiopian WEPs represents only about $5 \%$ of the country's districts indicating the need for further ethnobotanical research addressing unexplored regions of the country. Moreover, taking inspiration from the experience of other countries and conducting applied research on ecological distribution, nutritional analysis, toxicity, germplasm collection, promotion and domestication of WEPs is a timely endeavour to utilise potential food sources.

Many people in Ethiopia are undernourished due to factors related to economic, environmental and/or political problems. The availability and utilization of about $4 \mathrm{r} 3$ WEPs in the country, including those with high nutritional and market value, shows the food resource potential that could play a role in averting the recurrent undernourishment and also generate an economic gain.

Although about $4 \mathrm{I} 3$ WEPs have been investigated during the present work, this number could have increased considerably if all Ethiopian cultural groups had been addressed through ethnobotanical investigations. Ethiopian WEPs are faced with threats related to habitat loss and degradation; hence a complementary in situ and ex situ conservation measure is vital to conserve the WEP wealth of the country.

\section{Acknowledgements}

We would like to thank Mr. Desalegn Chala from Natural History Museum, Oslo University, Norway, and Mr. Wouter Vanhove from University of Ghent for their assistance in developing the map. Staff members of digital libraries of Economic Comission for Africa (ECA), Addis Ababa University (AAU), Ghent University (UGent) and the National Herbarium of Ethiopia (ETH) are also acknowledged for providing available resources on WEPs. 


\section{References}

Abate, D. (1999). Agaricus campestris in upland Ethiopia. Mycologist, 13:28.

Abbink, J. (1993). Me'en ritual, medicinal and other plants: A contribution to South-West Ethiopian Ethnobotany. Journal of Ethiopian Studies 26(2): I-2I.

Abdillahi, H., Stafford, G., Finnie, J., Staden, J. (2010). Ethnobotany, phytochemistry and pharmacology of Podocarpus sensu latissimo (S.I.). South Afr. J. Bot. 76(I): 1-24.

Aberoumand, A. (2009). Nutritional evaluation of edible Portulaca oleracea as plant food. Food Analyt. Meth. (2): 204-207.

Aberoumand, A. and Deokule, S. (2009). Studies on nutritional values of some wild edible plants from Iran and India. Pakistan Journal of Nutrition 8 (I): 26-3I.

Addis, G. (2006). Depth and breadth of collecting and documenting plant-use data through herbarium specimen labels and Ethiopian Flora volumes: the case of five Angiosperm families. Addis Ababa University.

Addis, G. (2009). Wild and Semi-wild edible plants of Hamar and Xonso (South Ethiopia) with emphasis on their ethnobotany and nutritional composition of selected species. $\mathrm{PhD}$ thesis, Addis Ababa University.

Addis, G., Urga, K. and Dikasso, D. (2005). Ethnobotanical study of edible wild plants in some selected districts of Ethiopia. Human Ecology 33(I): 83-1 8 .

Afolayan, A. and Jimoh, F. (2009). Nutritional quality of some wild leafy vegetables in South Africa. International Journal of Food Science and Nutrition 60 (5): 424-431.

Agea, J., Kabboggoza, J. and Waisaw, D. (2007). Diversity of indigenous fruit trees in the traditional cotton-millet farming system: the case of Adwari sub county, Lira district, Uganda. Afr. J. Ecol. (45): 39-43.

Awas, T. (2007). Plant diversity in Western Ethiopia: ecology, ethnobotany and conservation. PhD thesis, University of Oslo.

Arenas, P. and Scarpa, G. (2007). Edible wild plants of Chorote Indians, Gran Chacho, Argentina. Botanical Journal of the Linean Society 153: $73-85$.

Asfaw, Z. (1999). Ethnobotany of Nations, Nationalities and Peoples in Gambella, Benishangul-Gumuz and southern regions of Ethiopia. Research and Publication Office, Addis Ababa University.

- (2009). The future of wild food plants in southern Ethiopia: Ecosystem conservation coupled with enhancement of the roles of key social groups. Acta Horticulturae 806: 701-707.

Asfaw, Z. \& Tadesse, M. (200I). Prospects for sustainable use and development of wild food plants in Ethiopia. Econ. Bot. 55: 47-62.

Balemie, K. and Kibebew, F. (2006). Ethnobotanical study of wild edible plants in Derashe and Kucha Districts, South Ethiopia. Journal of Ethnobiology and Ethnomedicine 2006, 2: 53.

Bandyopadhyay, S. and Mukherjee, S. (2008). Wild edible plants of Koch Bihar district, West Bangal. Natural Product Radiance 8(I): 64-72.

Becker, B. $(1983)$. The contribution of wild plants to human nutrition in the Ferlo (North Senegal). Agroforestry Systems 1: 257-267.

Belcher, B., Ruı' z-Pe' rez, M. and Achidiawan, R. (2005). Global patterns and trends in the use and management of commercial NTFPs. World Dev. 33: 1435-1452.

Beluhan, S. and Ranogajec, A. (2010). Chemical composition and non-volatile components of Croatian wild edible mushrooms. Food Chemistry 124: 1076-1082.

Bharucha, Z. and Pretty, J. (2010). The roles and values of wild foods in agricultural systems. Phil. Trans. R. Soc. B. 365: 2913-2926.

Binu, S. (2010). Wild edible plants used by the tribals in Pathanmathitta district, Kerela. Indian Journal of Traditional Knowledge 9 (2): 309-312.

Boa, E. (2004). Wild edible fungi: a global overview of their use and importance to people. Non-Wood Forest Products, No. 17, FAO, Forestry Department, Rome, Italy.

Challe, J. and Struik, P. (2008). The impact on orchid species abundance of gathering their edible tubers by HIVIAIDS orphans: a case of three villages in the Southern Highlands of Tanzania. NJAS 56 (3): 26I-279. 
CI (2004). Hotspots Revised. (R. A. Mittermeier, P. R. Gil, M. Hoffman, J. Pilgrim, T. Brooks, C. G. Mittermeier, J. L. Gustavo and A. B. Fonseca. eds.) Conservation International. Cemex.

Clarke, C. B. (1977). Edible and useful plants of California. University of California Press. $280 \mathrm{pp}$.

Davenport, R. B., Ndangalasi, J. (2003). An escalating trade in orchid tubers across Tanzania's Southern Highlands: assessment, dynamics and conservation implications. Oryx 37(1) $55-6 \mathbf{I}$.

De Caluwé, E., Halamová, K., and Van Damme, P. (2010a). Adansonia digitata L. - A review of traditional uses, phytochemistry and pharmacology. Afrika Focus 23(I): II-5I.

(2010b). Tamarindus indica L. - A review of traditional uses, phytochemistry and pharmacology. Afrika Focus 23(I): $53-83$.

Degrande, A., Schreckenberg, K. and Mbosso, C. (2006). Farmers fruit tree growing strategies in the humid forest zone of Cameroon and Nigeria. Agroforest Syst 67: 159-175.

Delang, C. (2006). Economic valuation of non-marketed wild edible plants in Thailand. Environmental Conservation 32: $285-287$.

Edwards, S. B. (1991). Crops with wild relatives found in Ethiopia. In: Plant Genetic Resources of Ethiopia. (J.M.M. Engels, J.G. Hawkes and Melaku Werede, eds.). pp. 42-74. Cambridge University Press, Cambridge.

Ertug, F. (2004). Wild edible plants of the Bodrum Area (Mugla, Turkey). Turkish Journal of Botany 28: 161-174.

Fantahun, M. and Hager, H. (2010). Integration of indigenous wild woody perennial edible fruit bearing species in the agricultural landscapes of Amhara region. Agroforest Syst 78: 79-95.

Feyssa, D., H., Njoka, J, T., Asfaw, Z. and Nyangito, M. M. (201 I). Wild edible fruits of importance for human nutrition in semi-arid parts of east Shewa Zone, Ethiopia: Associated indigenous knowledge and implications to food security. Pakistan Journal of Nutrition Io (1): 40-50.

Freiberger, C., Vanderjagt, D., Pastuszyn, A., Glew, R. S, Mounkaila, G., Millson, M. and Glew, R. H. (1998). Nutrient content of the edible leaves of seven wild plants from Niger. Plant Foods Hum Nutr 53:5769 .

Gebauer, J., Patzelt, A., Hammer, K. and Buerkert, A. (2007). First record of Grewia tenax (Forssk.) Fiori in Northern Oman, a valuable fruit producing shrub. Genet Resour Crop Evol 54: 1153-1158.

Getahun, A. (1974). The role of wild plants in the native diet in Ethiopia. Agro. Ecosystems I: 45-56.

Ghirardini, M., Carli, M., del Vecchio, N., Rovati, A., Cova, A., Valigi, A., Agnetti, G., Macconi. M., Adamo, D., Traina, M., Laudini, F., Marcheselli, I., Caruso, N., Gedda, T., Donati, F., Marzadro, A., Russi, P., Spaggiari, C., Bianco, M., Binda, R., Barattieri, E., Tognacci, A., Girardo, M., Vaschetti, L., Caprino, P., Sesti, E., Andreozzi, G., Coletto, E., Belzer, G. and Pieroni, A. (2007). The importance of a taste. A comparative study on wild food plant consumption in twenty-one local communities in Italy. Journal of Ethnobiology and Ethnomedicine 2007, 3: 22.

Giday, M., Asfaw, Z. and Woldu, Z. (2009). Medicinal plants of the Meinit ethnic group of Ethiopia: An ethnobotanical study. J Ethnopharmacol 124 (3): 513-521.

Glew, S., Vanderjagt, D., Chuang, L., Huang, Y., Millson, M., and Glew, R. (2005). Nutrient content of four edible wild plants from West Africa. Plant Foods for Human Nutrition 60 (4): I87-193.

Glew, R., Kramer, J., Hernandez, M., Pastuszyn, A., Ernst, J., Djmodi, N., Vanderjagt, D. (2010). The amino acid, mineral and fatty acid content of three speciesof human plant foods in Cameroon. Food (4) I: I-6.

Grivetti, L. and Ogle, B. (2000). Value of traditional foods in meeting macro and micronutrient needs: the wild plant connection. Nutr. Res. Rev. 13: 31-46.

Grosskinsky, B. and Gullick, C. (eds.) (1999). Exploring the potential of wild food plants in Southern Sudan. Proceedings of a workshop held in Lokichoggio, Kenya, June 3-5, 1999.

Guill-Guerrero, J. and Rodríguez-García, I. (1999). Lipids classes, fatty acids and carotenes of the leaves of six edible wild plants. Eur Food Res Technol 209:313-316.

Guill-Guerrero, J., Rodríguez-García, I. and Torija, E. (1997). Nutritional and toxic factors in selected wild edible plants. Plant Foods for Human Nutrition 5I(2): 99-107. 
Guinand, Y. and Lemessa, D. (2000). Wild-food plants in Southern Ethiopia: Reflections on the role offamine-foods at a time of drought. UNDP-EUE. Field mission report, Addis Ababa, 2000.

Hadjichambis, A., Hadjichambis, D., Della, A., Elena, M., de Pasquale, C., Lenzarini, C., Censorii, E., Tejero, M., Rojas, C., Gutierrez, J., Skoula, M., Johnson, C., Sarpaki, A., Hmamouchi, M., Jorhi, S., El-Demerdash, M., El-Zayat, M., and Pieroni, A. (2008). Wild and semi-domesticated food plant consumption in seven circum-Mediterranean areas. International Journal of Food Sciences and Nutrition 59(5): 383-414.

Hedberg, I., Friis, I., Person, E. (eds.) (2009). General Part and Index to Vol I-7. Flora of Ethiopia and Eritrea Volume 8. The National Herbarium, Addis Ababa, Ethiopia and Uppsala, Sweden.

Heinrich, M., Leonti, M., Nebel, S. and Peschel, W. (2005). 'Localfood-nutraceuticals': an example of a multidisciplinary research project on local knowledge. Journal of Physiology and Pharmacology 56: 5-22.

Http://database.prota.org Useful plants of tropical Africa, Accessed on 26 July 2011.

Jaenicke, H. and Hoschle-Zeledon, I. (eds.) (2006). Strategic framework for underutilized plant species research and development. Rome, Italy: ICUC, Colombo and Global Facilitation Unit for Underutilized Species.

Johns, T., Mohoro, E. B. and Sanaya, P. (1996). Food plants and masticants of the Batemi of Ngorongoro District, Tanzania. Econ. Bot. 50: I15-121.

Ryan, K. (2000). Edible plants as digestive aids. Expedition 42(3): 7-8.

Kelbessa, E., Demissew, S., Woldu, Z. and Edwards, S. (1992). Some threatened Endemic Plants of Ethiopia. In: The status of some plants in parts of tropical Africa (Edwards, S. and Asfaw Z. eds.). NAPRECA, No. 2. Botany 2000: East and Central Africa, pp. 35-55.

Lockett, C. and Grivetti, L. (2000). Food related behaviours during drought: a study of rural Fulani, North-eastern Nigeria. International Journal of Food Science and Nutrition 51: 91-107.

Loghrust, R. (1986). Household food strategies in response to seasonality and famine. IDS Bulletin 17: 27-35.

Lopez-Garcia, S. and Basurto-Pena, F. (2007). Content of nutrient and Antinutrient in Edible Flowers of Wild Plants in Mexico. Plant Foods Hum Nutr 62: 133-138.

Luczaj, L. (2010). Changes in the utilization of wild green vegetables in Poland since 19th century: A comparison of four ethnobotanical surveys. Journal of Ethnopharmacology I28: 395-404.

Lulekal, E. (2009). Ethnobotanical study of four important Angiosperm families (Rubiaceae, Solanaceae, Cucurbitaceae, and Burseraceae) based on the collections at the National Herbarium of Ethiopia and their accounts in the Flora of Ethiopia and Eritrea, Addis Ababa University.

Martin, G. J. (1995). Ethnobotany: A method Manual. Chapman and Hall, London. 268 pp.

Mazhar, F., Buckles, D., Satheesh, P. V. and Akhter, F. (2007). Food sovereignty and uncultivated biodiversity in South Asia. Academic Foundation. New Delhi, India. $84 \mathrm{p}$.

Misra, S., Maikhuri, R., Kala, C., Rao, K. and Saxena, K. (2008). Wild leafy vegetables: a study of their subsistence dietetic support to the inhabitants of Nanda Devi Biosphere Reserve, India. Journal of Ethnobiology and Ethnomedicine 2008, 4: 15 .

Mothanka, D. M, Motlhanka, P. and Selbatso, T. (2008). Edible indigenous Wild fruit Plants of Eastern Botswana. International Journal of Poultry Science 7 (5): 57-460.

Ogle, B. M. and Grivetti, L. E. ( 1985$)$. Legacy of the chameleon edible wild plants in the kingdom of Swaziland, South Africa. A cultural, ecological and nutritional study. Part II-IV, species availability and dietary use, analysis by ecological zone. Ecology of Food and Nutrition 17: 1-30.

Ogle, B. M., Tuyet, H. T., Duyet, H. N. and Dung, N. X. (2003). Food, feed or medicine: the multiple functions of edible wild plants in Vietnam. Econ. Bot. 57: 103-117.

Ohiokpehal, O. (2003). Promoting the nutritional goodness of traditional food products. Pakistan Journal of Nutrition 2 (4): 267-270.

Okaraonye, C. and Ikewuchi, J. (2009). Nutritional and antinutritional components of Pennisetum purpureum (Schumach). Pakistan Journal of Nutrition 8(I): 32-34. 
Pieroni, A., Nebel, S., Quave, C., Münz, H., Heinrich, M. (2002). Ethnopharmacology of Liakra: traditional weedy vegetables of the Arbëreshe of the Vulture area in southern Italy. Journal of Ethnopharmacology 81: $165-185$.

Pilgrim, S., Cullen, L., Smith, D. J. and Pretty, J. (2008). Ecological knowledge is lost in wealthier communities and countries. Environ. Sci. Tech. 42: 1004-1009.

Rashid, A., Anand, V. K. and Serwar, J. (2008). Less known wild edible plants used by the Gujjar tribe of district Rajouri, Jammu and Kashmir State. Int. J. Bot. 4: 219-224.

Rathore, M. (2009). Nutrient content of important fruit trees from arid zone of Rajasthan. J. Hort. Forestry I: 103-108.

Reyes-Garcia, V., Vadez, V., Huanca, T., Leonard, W. and Wilkie, D. (2005). Knowledge and consumption of wild plants: A comparative study in two Tsimane villages in the Bolivian Amazon. Ethnobotany Research and Applications 3: 201-207.

Shrestha, P.M. and Dhillon, S.S. (2006). Diversity and traditional knowledge concerning wild food species in a locally managed forest in Nepal. Agroforest. Syst. 66: $55-63$.

Somnasang, P. and Moreno-Black, G. (2000). Knowing, gathering and eating: knowledge and attribute about wild food in an Asian village in North-eastern Thailand. J. Ethnobiol. 20: 197-216.

Spina, M., Cuccioloni, M., Sparapani, L., Acciarri, S., Eleuteri, A., Fioretti, E. and Angeletti, M. (2008). Comparative evaluation of flavonoid content in assessing quality of wild and cultivated vegetables for human consumption. Journal of the Science of Food and Agriculture 88: 294-304.

Tabuti, J. (2007). Status of non-cultivated food plants in Bulamogi County, Uganda. African Journal of Ecology 45 (Suppl. I), 96-10r.

Tardio, J., Pardo-de-Santayana, M., and Morales , R. (2006). Ethnobotanical review of wild edible plants in Spain. Botanical Journal of the Linnean Society I52: 27-7I.

Teketay, D., Senbeta, F., Maclachlan, M., Bekele, M. and Barklund, P. (2010). Edible Wild Plants in Ethiopia. Addis Ababa University Press, 575pp.

Teklehaymanot, T. and Giday, M. (2010). Ethnobotanical study of wild edible plants of Kara and Kewego semipastoralist people in Lower Omo River valley, Debub Omo Zone, SNNPR, Ethiopia. Journal of Ethnobiology and Ethnomedicine 2010, 6: 23.

Termote, C., Meyi, M., Ndjango, J., Van Damme, P. and Dhed'a Djailo, B. (2009). Use and socioeconomic importance of wild edible plants in tropical rainforest around Kisangani district, Tshopo, DR Congo. In: Systematics and conservation of African plants (X. Van der Burgt, J. Van der Maesen and J. M. Onana eds.). pp. 415-425. Royal Botanic Gardens, Kew.

Termote, C., Van Damme, P. and Dhed'a Djailo, B. (2010). Eating from the wild. Turumbu indigenous knowledge on non-cultivated edible plants, district Tshopo, DR. Congo. Ecology of Food and Nutrition, 49:3, $173-207$.

(20II). Eating from the wild. Turumbu, Mbole and Bali traditional knowledge on non-cultivated edible plants, Tshopo district, DR. Congo. Ecology of Food and Nutrition, 49:3, 173-207.

Tuno, N. (2001). Mushroom utilization by the Majangir, an Ethiopian tribe. The Mycologist, 15: 78-79.

Turan, M., Kordali, S., Zengin, H., Dursun, A. and Sezen, Y. (2003). Macro and micro mineral content of some wild edible leaves consumed in Eastern Anatolia. Acta Agric. Scand., Sect. B, Soil and Plant Sci. 2003, 53: 129-137.

Van Damme, P. and Termote, C. (2008). African botanical heritage for new crop development. Afrika Focus: 2I(I): $45-64$.

Vanderjagt, D., Freiberger, C., Mounkaila, G., Glew, R.S., and Glew, R.H. (2000). The Trypsin inhibitor content of 61 wild edible plant foods of Niger, Plant Foods for Human Nutrition 55: 335-346.

Wondimu, T., Asfaw, Z., and Kelbessa, E. (2006). Ethnobotanical study of food plants around Dheeraa Town, Arsi, Ethiopia. SINET: Ethiop. J. Sci. 29:71-80 


\section{Appendix}

Appendix 1 Wild edible plants of Ethipia

Abbreviations and Symbols Habit: $\mathrm{T}=$ tree, $\mathrm{S}=\mathrm{shrub}, \mathrm{H}=\mathrm{herb}, \mathrm{C}=\mathrm{Climber}$. Local names: Afa-Afar, Amh-Amharic, Anu-Anuak, Awi-Awi, Ben-Bena, Bench-Bench, BerBerta, D-Derashe, G-Gamo, Gum-Gumuz, Gur-Gurage, Had-Hadiya, Ham-Hamar KKusume, KA-Kara, Kaf-Kafficho, Kon-Xonso, KW-Kwego, NM-Not mentioned, Maj-Majanjir, Mur-Mursi, Nue-Nuer, Oro-Affan Oromo, She-Sheko, Sid-Sidamo, Som-Somali, Tig-Tigray, Tse-Tsemay, Wel- Welaita, Zay-Zay. Source: I- Balemie and Kibebew, 2006, 2- Teklehaymanot and Giday, 2010, 3- Teketay et al., 2010, 4- Addis et al., 2005, 5- Fantahun and Hager, 20ro, 6- Guinand and Lemessa, 2000, 7- Lulekal, 2009, 8- Addis, 2006, 9- Addis, 2009, I0- Asfaw \& Tadesse, 2001, II- Wondimu et al., 2006, I2- Awas, 2007, I3- Asfaw, I999. 


\begin{tabular}{|c|c|c|c|c|c|c|c|c|c|c|c|c|c|c|c|}
\hline 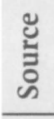 & $\stackrel{ }{\circ}$ & $m$ & in & $\hat{m}$ & $n$ & $m$ & $\begin{array}{l}\tilde{n} \\
\dot{n}\end{array}$ & $\hat{m}$ & $\begin{array}{l}\tilde{n} \\
\dot{n} \\
\dot{n}\end{array}$ & t & $m$ & $n$ & $\stackrel{m}{\sim}$ & + & $m$ \\
\hline 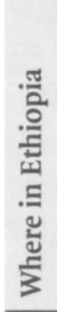 & 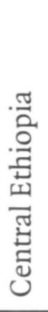 & 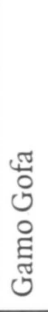 & $\begin{array}{l}\text { 棓 } \\
\text { 营 } \\
\end{array}$ & 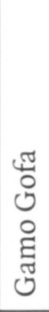 & 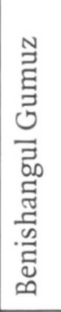 & 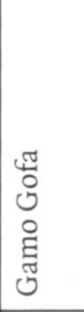 & 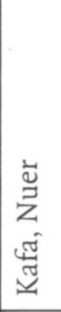 & 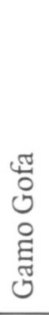 & 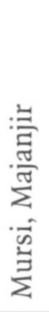 & 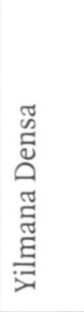 & 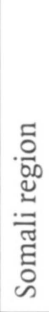 & 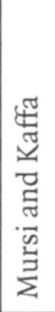 & 光 & 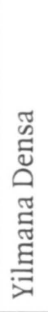 & 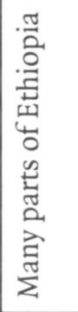 \\
\hline 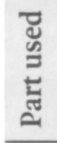 & 志 & 芯 & 声 & 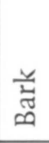 & 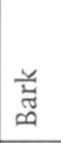 & 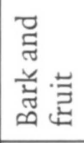 & 当 & 芯 & 壱 & ఏ్ & 壱 & 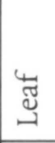 & 崩 & $\begin{array}{l}\text { 芯 } \\
\text { ž }\end{array}$ & 鸪 \\
\hline 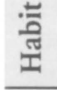 & $\mapsto$ & $H$ & $H$ & $H$ & $H$ & $H$ & $H$ & $H$ & $H$ & $r$ & $t$ & $H$ & is & in & is \\
\hline 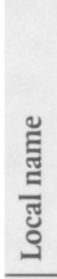 & 莺 & 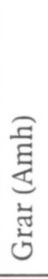 & 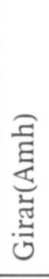 & 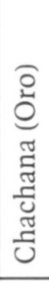 & 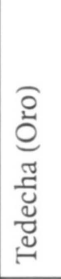 & 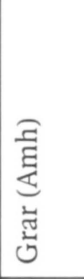 & 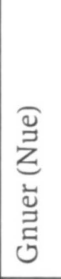 & 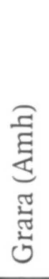 & 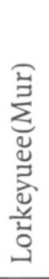 & 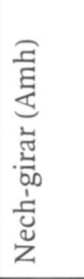 & 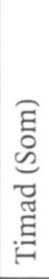 & 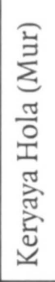 & 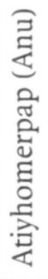 & 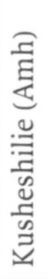 & 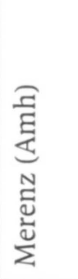 \\
\hline 를 & $\begin{array}{l}\stackrel{\mathscr{J}}{\sharp} \\
\stackrel{\widetilde{J}}{0} \\
\text { त्ञ }\end{array}$ & 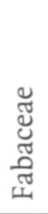 & 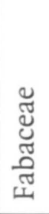 & 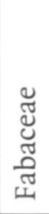 & 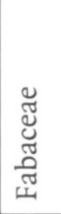 & 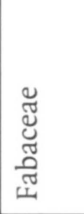 & 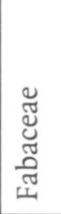 & 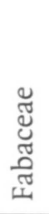 & 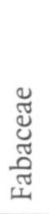 & 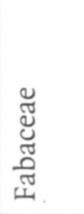 & 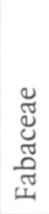 & 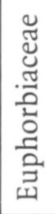 & 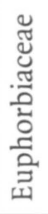 & 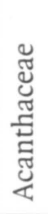 & 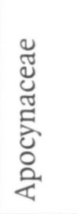 \\
\hline 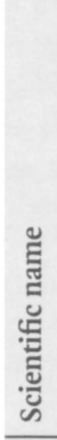 & 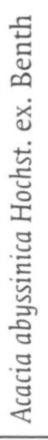 & 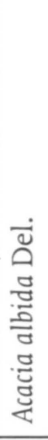 & 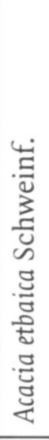 & 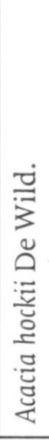 & 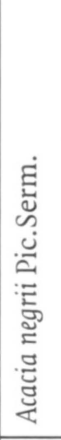 & 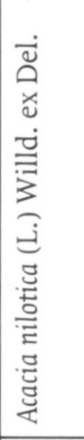 & 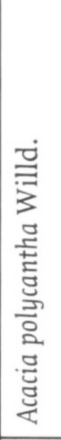 & 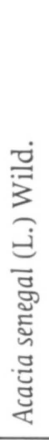 & 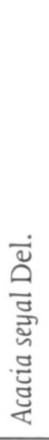 & 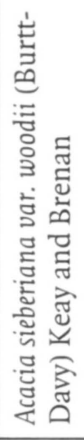 & 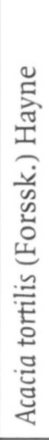 & 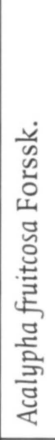 & 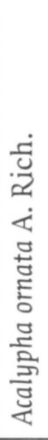 & 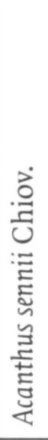 & 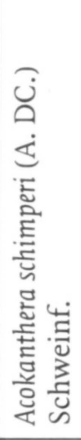 \\
\hline z & $\rightarrow$ & $N$ & $m$ & + & in & 0 & $\Lambda$ & $\infty$ & $a$ & 윽 & $\Xi$ & $\simeq$ & $\stackrel{m}{\sim}$ & t & $\cong$ \\
\hline
\end{tabular}




\begin{tabular}{|c|c|c|c|c|c|c|c|c|c|c|c|c|c|}
\hline 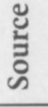 & $\stackrel{\dot{\rho}}{\dot{m}} m$ & $m$ & $a$ & $\stackrel{n}{\sim}$ & $\stackrel{n}{\sim}$ & + & $m$ & $\stackrel{m}{i}$ & $\begin{array}{l}a \\
\hat{n} \\
i\end{array}$ & $\hat{n}$ & $n$ & $\stackrel{m}{i}$ & $\stackrel{m}{n}$ \\
\hline 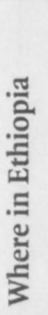 & 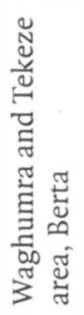 & 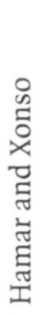 & 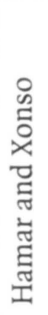 & 胥 & 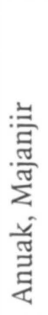 & 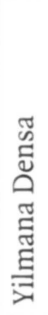 & 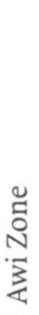 & 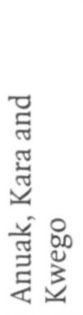 & 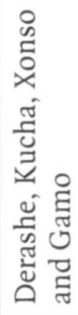 & 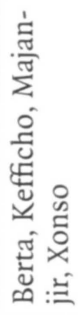 & 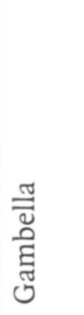 & 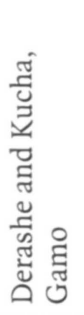 & 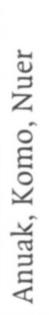 \\
\hline 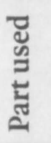 & 莺 & $\stackrel{4}{\mathbb{G}}$ & 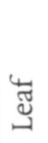 & 总 & 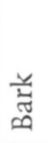 & 当 & 莺 & 壱 & $\begin{array}{l}\ddot{\Xi} \\
\ddot{\sim}\end{array}$ & 苂 & 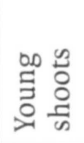 & 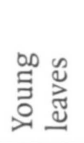 & 苂 \\
\hline 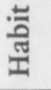 & $H$ & I & $u$ & $I$ & $H$ & $H$ & $H$ & $H$ & $I$ & $I$ & $I$ & $I$ & $I$ \\
\hline 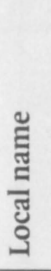 & 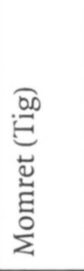 & 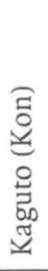 & 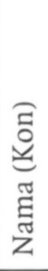 & 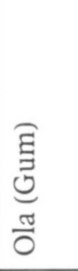 & 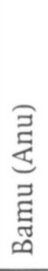 & 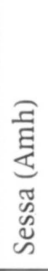 & 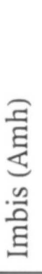 & 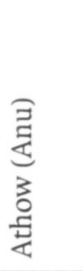 & 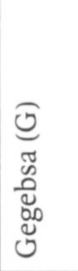 & 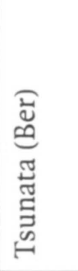 & 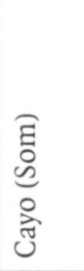 & 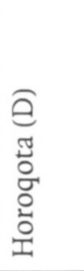 & 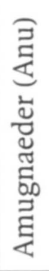 \\
\hline 离 & 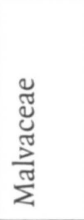 & 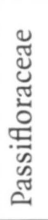 & 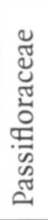 & 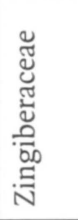 & 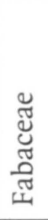 & 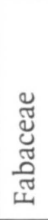 & 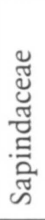 & 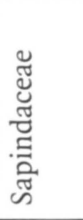 & 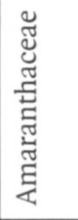 & 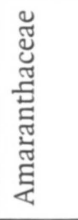 & 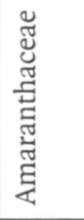 & 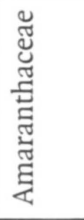 & 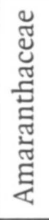 \\
\hline 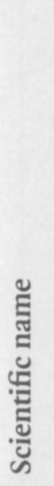 & 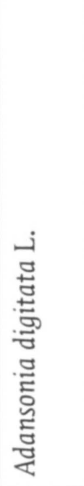 & 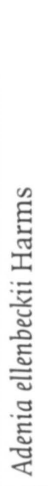 & 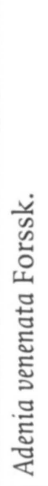 & 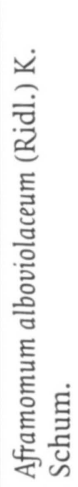 & 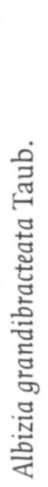 & 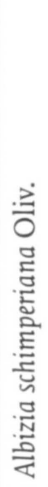 & 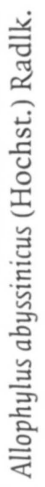 & 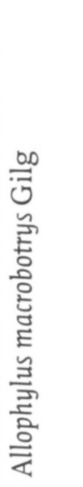 & 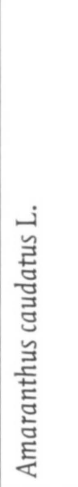 & 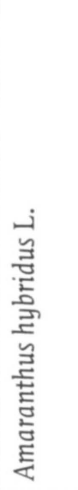 & 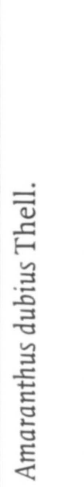 & 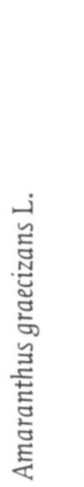 & 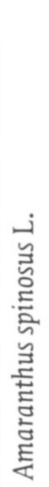 \\
\hline z & 이 & 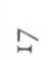 & $\stackrel{\infty}{\infty}$ & 9 & กे & $\vec{N}$ & ה & $\tilde{N}$ & a & $\stackrel{\sim}{N}$ & $m_{0}^{4}$ & $\hat{\lambda}$ & $\mathbb{N}^{-1}$ \\
\hline
\end{tabular}




\begin{tabular}{|c|c|c|c|c|c|c|c|c|c|c|c|c|c|}
\hline 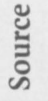 & $m$ & $m$ & $\stackrel{n}{\tilde{0}}$ & $a$ & $m$ & $\begin{array}{l}n \\
\stackrel{n}{0}\end{array}$ & 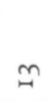 & $m$ & $\begin{array}{l}\dot{0} \\
i \\
i\end{array}$ & $m$ & $a$ & $\hat{m}$ & $\stackrel{\sim}{\sim}$ \\
\hline 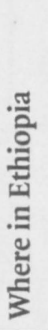 & 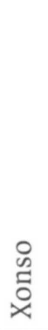 & $\begin{array}{l}0 \\
\infty \\
0 \\
\varnothing \\
\varnothing\end{array}$ & 光 & 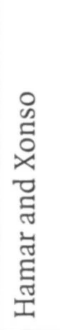 & 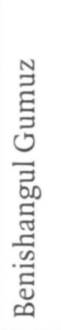 & 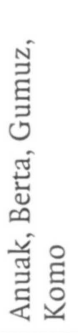 & : & 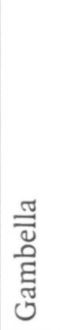 & 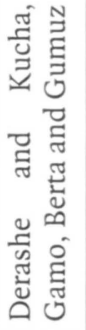 & $\sum_{\Sigma}^{Z}$ & 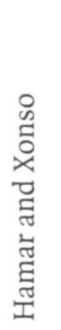 & 孞 & ్ㅠ \\
\hline 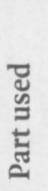 & $\begin{array}{l}0 \\
0 \\
0 \\
\frac{0}{\omega} \\
0 \\
\Xi \\
\vdots \\
0\end{array}$ & $\stackrel{\breve{\Xi}}{\stackrel{\Xi}{\Xi}}$ & 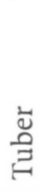 & $\begin{array}{l}\text { मे } \\
\stackrel{2}{2}\end{array}$ & 壱 & 莺 & 莺 & 㫕 & 壱 & 莺 & జ్̈ & $\begin{array}{l}\stackrel{\breve{\nu}}{ٍ ٍ ~} \\
\stackrel{\Xi}{\Xi}\end{array}$ & 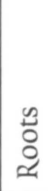 \\
\hline 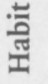 & I & I & $I$ & I & I & 0 & $H$ & 工 & $H$ & 工 & I & $I$ & I \\
\hline 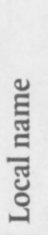 & 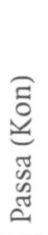 & 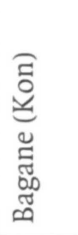 & 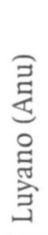 & 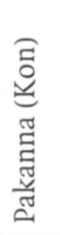 & 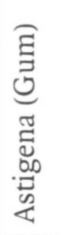 & 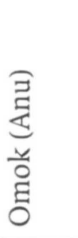 & 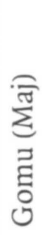 & 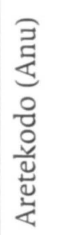 & 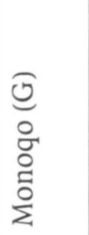 & 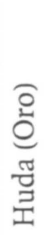 & 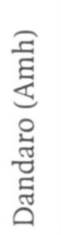 & 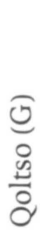 & 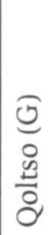 \\
\hline 﨎 & 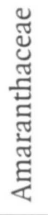 & 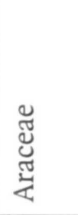 & 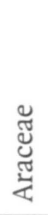 & 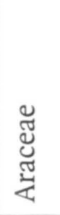 & 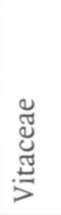 & 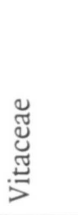 & 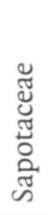 & 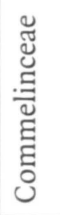 & 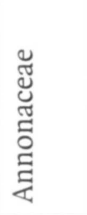 & 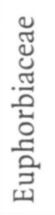 & 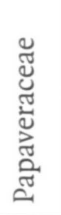 & 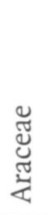 & 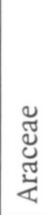 \\
\hline 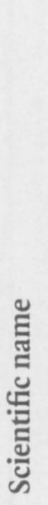 & 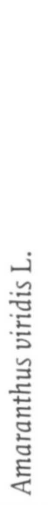 & 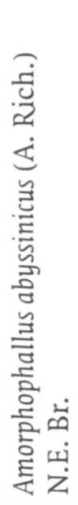 & 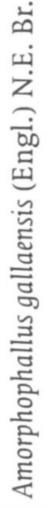 & 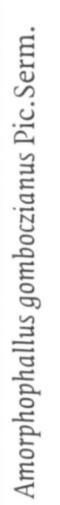 & 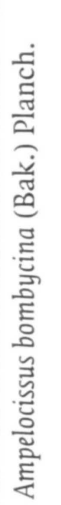 & 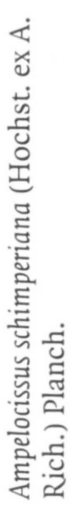 & 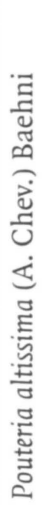 & 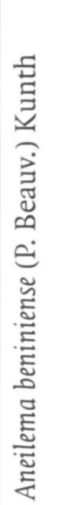 & 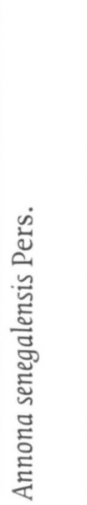 & 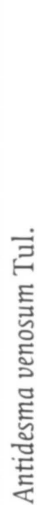 & 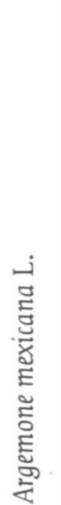 & 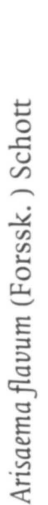 & 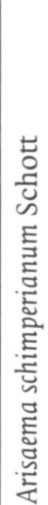 \\
\hline ż & నิ & $\stackrel{\circ}{m}$ & $\bar{m}$ & $\stackrel{n}{m}$ & $m$ & 市 & $\stackrel{n}{m}$ & $\stackrel{m}{m}$ & $\hat{m}$ & $\stackrel{\infty}{m}$ & ले & o & F \\
\hline
\end{tabular}




\begin{tabular}{|c|c|c|c|c|c|c|c|c|c|c|c|c|}
\hline 苟 & $m$ & $a$ & $\begin{array}{l}q \\
\dot{f}\end{array}$ & $\begin{array}{l}m \\
a\end{array}$ & $\begin{array}{l}\hat{0} \\
\hat{a} n \\
i n\end{array}$ & $\begin{array}{l}\hat{a} \\
\hat{i} \\
\hat{i}\end{array}$ & $a$ & $a$ & $a$ & $m$ & $\begin{array}{l}0 \\
\dot{a} \\
\dot{n}\end{array}$ & + \\
\hline 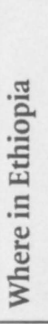 & 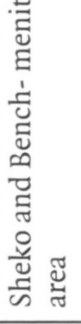 & 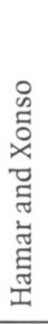 & 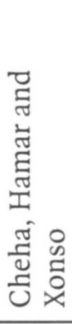 & 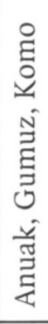 & 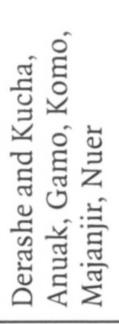 & 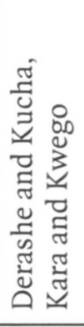 & 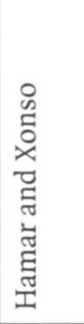 & 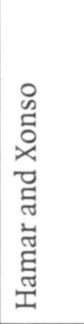 & 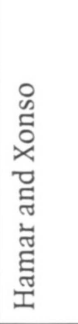 & 常 & 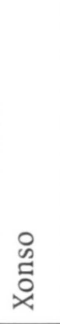 & $\begin{array}{l}\frac{\pi}{2} \\
\frac{\pi}{0}\end{array}$ \\
\hline 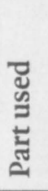 & 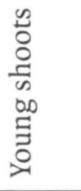 & 芯 & 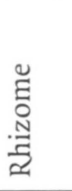 & 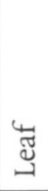 & 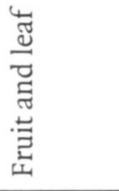 & 莺 & 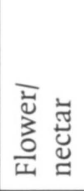 & 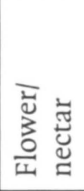 & 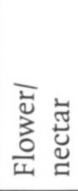 & 吉 & 鸪 & 怨 \\
\hline $\begin{array}{l}\text { 节 } \\
\text { 荧 }\end{array}$ & $H$ & is & n & I & $H$ & in & is & in & is & is & $H$ & I \\
\hline 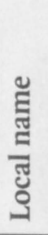 & 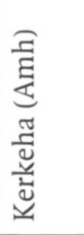 & 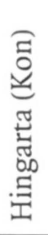 & 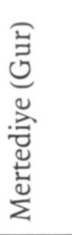 & 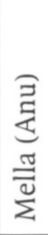 & 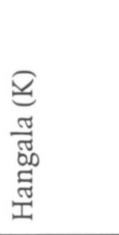 & 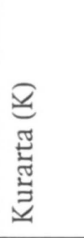 & 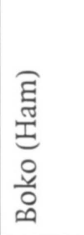 & 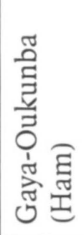 & 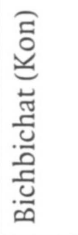 & 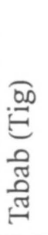 & 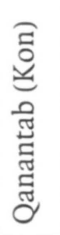 & 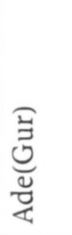 \\
\hline 㙳 & 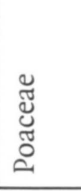 & 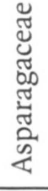 & 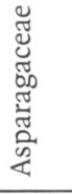 & 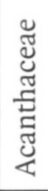 & 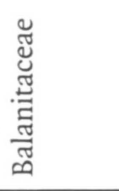 & 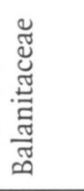 & 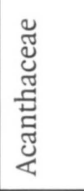 & 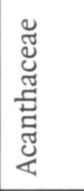 & 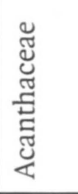 & 芯 & 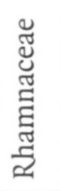 & 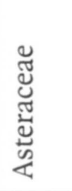 \\
\hline 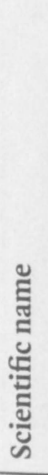 & 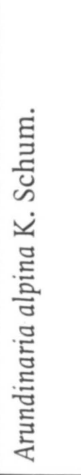 & 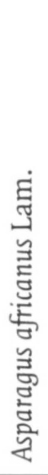 & 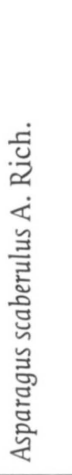 & 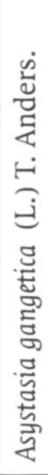 & 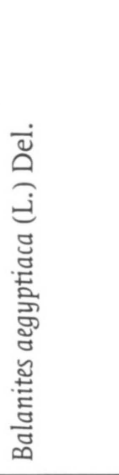 & 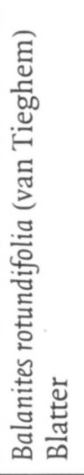 & 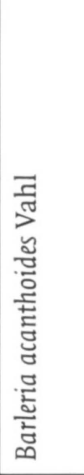 & 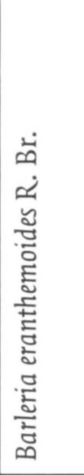 & 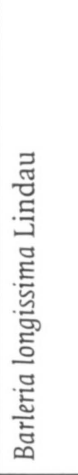 & 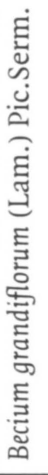 & 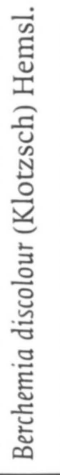 & 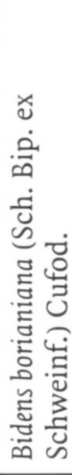 \\
\hline ż & Y & $\tilde{q}$ & f & 年 & + & f & $\stackrel{\infty}{+}$ & g & in & เท & กn & $n$ \\
\hline
\end{tabular}




\begin{tabular}{|c|c|c|c|c|c|c|c|c|c|c|c|c|}
\hline 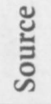 & $m$ & $m$ & 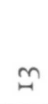 & $a$ & $\begin{array}{l}n \\
\hat{n} \\
i\end{array}$ & $\hat{a}$ & $a$ & $m$ & $\Lambda$ & $\stackrel{n}{i}$ & $\begin{array}{l}\dot{0} \\
\dot{a} n\end{array}$ & $m$ \\
\hline 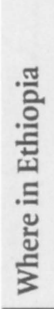 & $\begin{array}{l}\mathbb{\widetilde { T }} \\
\stackrel{\mathbb{I}}{\Xi}\end{array}$ & 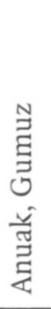 & 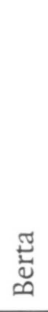 & 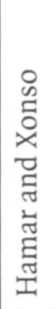 & 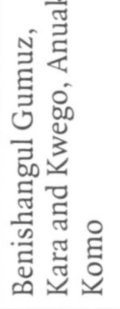 & 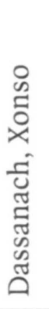 & 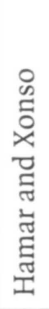 & 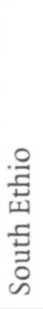 & 兰 & 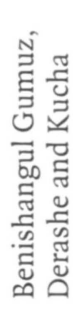 & 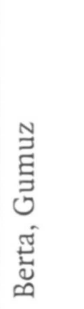 & 要 \\
\hline 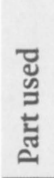 & $\stackrel{\widetilde{J}}{\leftrightarrows}$ & 亗 & $\underset{\Xi}{\mathbb{G}}$ & 总 & 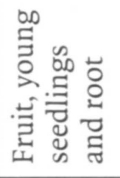 & 总 & $\stackrel{\widetilde{J}}{\leftrightarrows}$ & 壱 & 吾 & 总 & 壹 & 总 \\
\hline 节 & I & I & I & in & $\mapsto$ & en & $r$ & is & 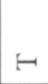 & is & is & is \\
\hline 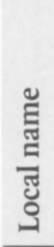 & 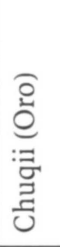 & 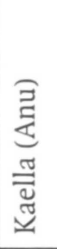 & 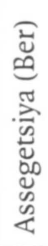 & 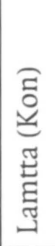 & 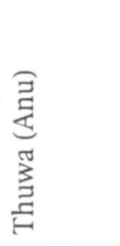 & 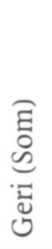 & 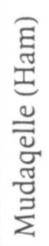 & 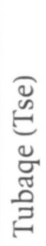 & 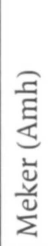 & 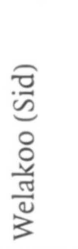 & 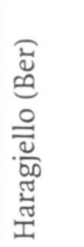 & 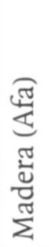 \\
\hline 哣 & 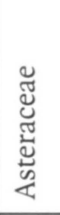 & 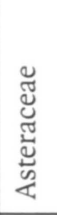 & 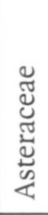 & 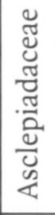 & 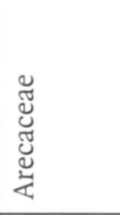 & 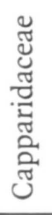 & 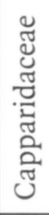 & 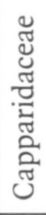 & 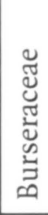 & 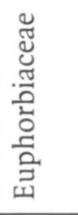 & 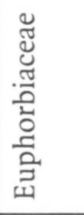 & 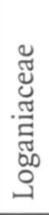 \\
\hline 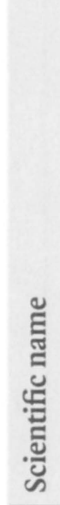 & 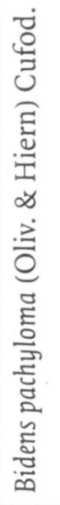 & 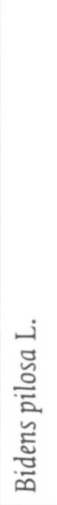 & 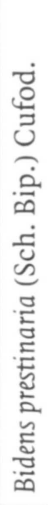 & 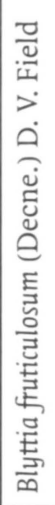 & 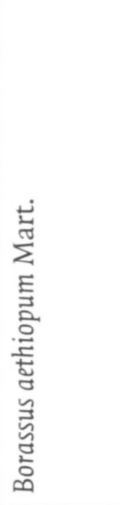 & 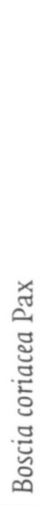 & 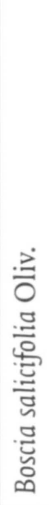 & 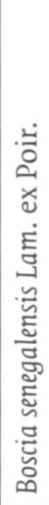 & 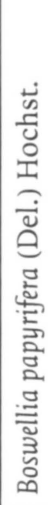 & 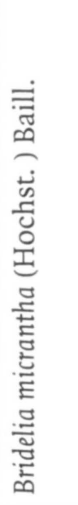 & 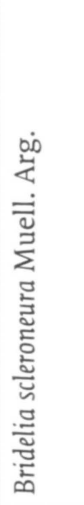 & 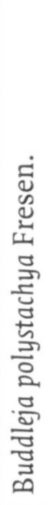 \\
\hline z & in & in & in & in & in & in & 8 & $\vec{b}$ & ถี & $\tilde{\sigma}$ & J & $\tilde{b}$ \\
\hline
\end{tabular}




\begin{tabular}{|c|c|c|c|c|c|c|c|c|c|c|c|c|c|}
\hline 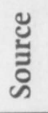 & $\cong$ & $\begin{array}{l}n \\
\dot{n} \\
\dot{i}\end{array}$ & $\Lambda$ & $\hat{m}$ & $m$ & $\stackrel{m}{\sim}$ & $ت$ & $m$ & 0 & $m$ & $m$ & $\Lambda$ & $\stackrel{\circ}{i}$ \\
\hline 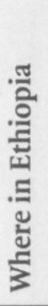 & 泾 & 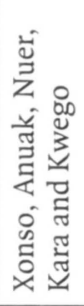 & 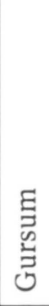 & 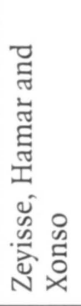 & $\begin{array}{l}\stackrel{0}{\circ} \\
3\end{array}$ & 莺 & 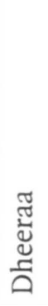 & 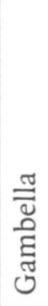 & 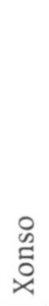 & 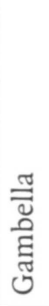 & 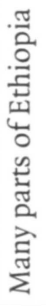 & 莺 & 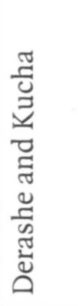 \\
\hline 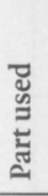 & 墨 & 宔 & 总 & 叴 & 坣 & 坣 & 莺 & 㕦 & 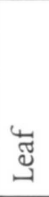 & $\begin{array}{l}\text { a } \\
0 \\
0 \\
\frac{1}{\infty} \\
0 \\
0 \\
\Xi \\
0\end{array}$ & 莺 & 苐 & 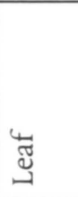 \\
\hline $\begin{array}{l}\text { : } \\
\text { 荧 }\end{array}$ & $H$ & is & is & is & $\infty$ & is & is & $\infty$ & is & I & is & $r$ & I \\
\hline 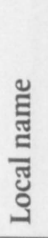 & 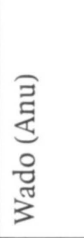 & 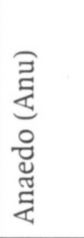 & 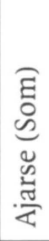 & 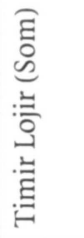 & 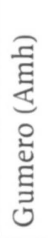 & 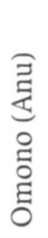 & 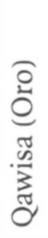 & 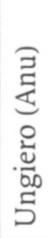 & 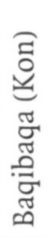 & 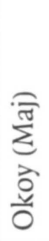 & 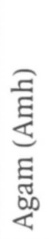 & 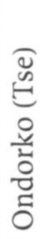 & 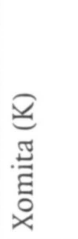 \\
\hline 䘚 & 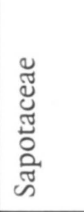 & 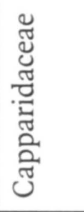 & 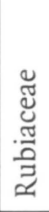 & 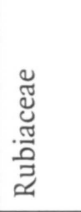 & 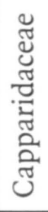 & 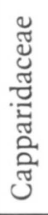 & 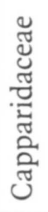 & 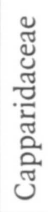 & 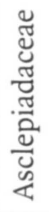 & 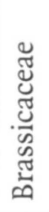 & 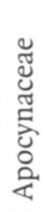 & 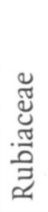 & 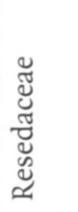 \\
\hline 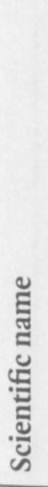 & 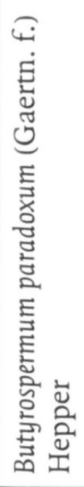 & 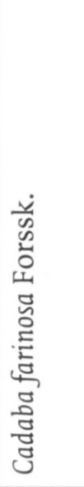 & 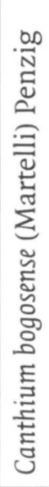 & 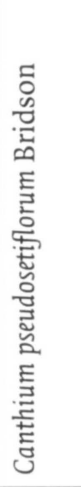 & 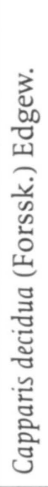 & 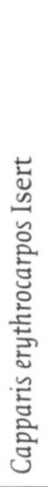 & 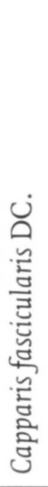 & 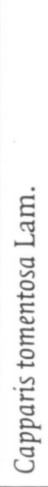 & 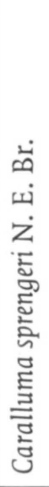 & 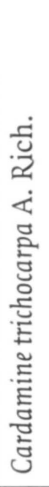 & 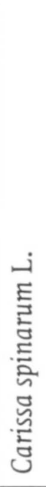 & 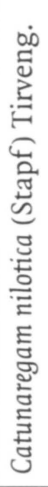 & 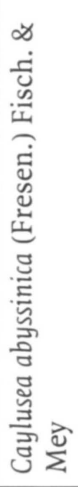 \\
\hline z & 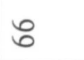 & $\hat{\sigma}$ & $\infty$ & ๑) & $N$ & $\vec{\wedge}$ & 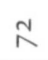 & $n$ & $\stackrel{+}{N}$ & $\stackrel{n}{n}$ & $\stackrel{\circ}{1}$ & $\hat{\wedge}$ & $\stackrel{\infty}{\wedge}$ \\
\hline
\end{tabular}


E. LULEKAL, Z. ASFAW, E. KELBESSA, P. VAN DAMME

\begin{tabular}{|c|c|c|c|c|c|c|c|c|c|c|c|c|c|}
\hline 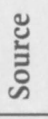 & $m$ & $a$ & $a$ & $\stackrel{m}{n}$ & $\begin{array}{l}n \\
i \\
i\end{array}$ & $\begin{array}{l}\hat{n} \\
\hat{n} \\
\hat{i}\end{array}$ & $\stackrel{m}{\sim}$ & $\infty$ & $a$ & 음 & $\stackrel{m}{\sim}$ & $a$ & $\stackrel{m}{i}$ \\
\hline 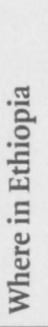 & 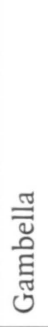 & 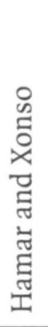 & 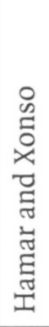 & 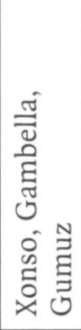 & 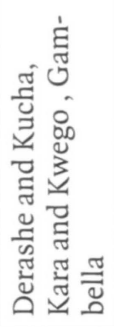 & 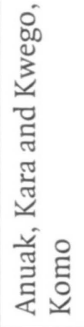 & 莺 & 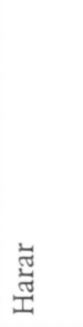 & 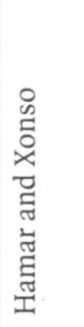 & 苞 & 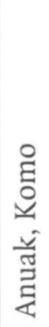 & 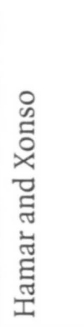 & 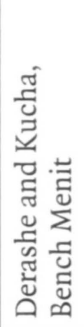 \\
\hline 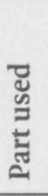 & 岌 & 亗 & $\stackrel{4}{\overparen{G}}$ & $\begin{array}{l}0 \\
0 \\
0 \\
0 \\
0 \\
0 \\
0 \\
\Xi \\
\Xi \\
0\end{array}$ & 莺 & 鸪 & 壱 & 莺 & 莺 & 㤩 & 志 & 鸪 & 莺 \\
\hline $\begin{array}{l}\text { 䓂 } \\
\text { 垔 }\end{array}$ & I & I & I & $I$ & $\mapsto$ & $\infty$ & is & is & u & 0 & 0 & $u$ & is \\
\hline 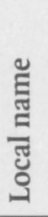 & 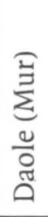 & 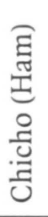 & 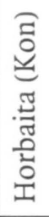 & 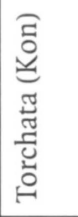 & 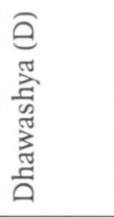 & 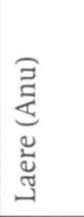 & 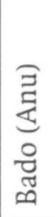 & $\sum_{Z}$ & 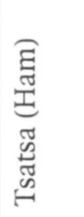 & 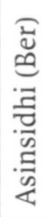 & 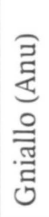 & 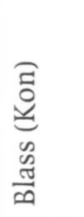 & 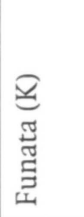 \\
\hline 层 & 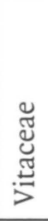 & 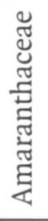 & 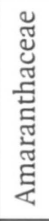 & 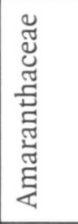 & 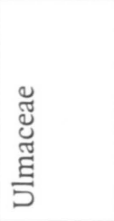 & 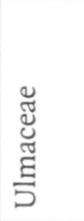 & 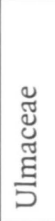 & 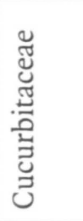 & 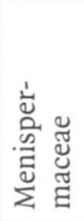 & 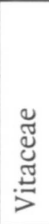 & 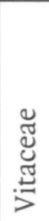 & 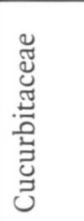 & 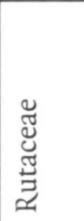 \\
\hline 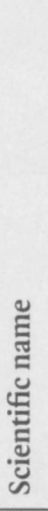 & 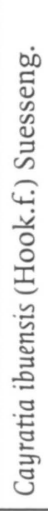 & 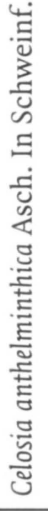 & 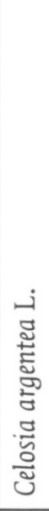 & 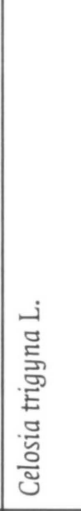 & 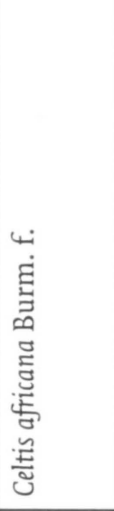 & 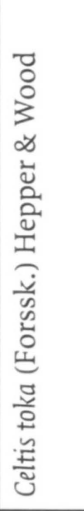 & 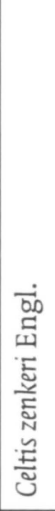 & 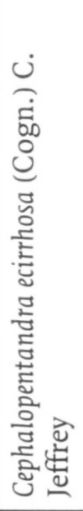 & 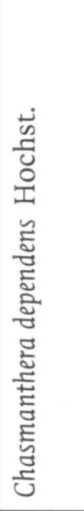 & 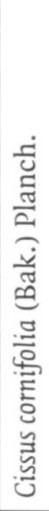 & 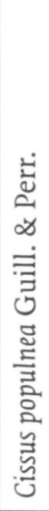 & 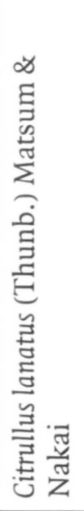 & 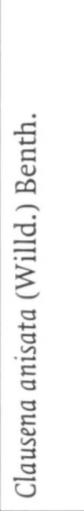 \\
\hline ¿ & r & $\infty$ & $\infty$ & $\infty$ & $\infty$ & ఉా & $\infty$ & $\infty$ & $\infty$ & $\infty$ & ১) & ஃ & ప \\
\hline
\end{tabular}




\begin{tabular}{|c|c|c|c|c|c|c|c|c|c|c|c|c|c|}
\hline 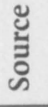 & $a$ & $\nabla$ & $\begin{array}{l}\dot{n} \\
\hat{i}\end{array}$ & $m$ & $m$ & $m$ & $\stackrel{n}{0}$ & $\begin{array}{l}\dot{m} \\
\hat{i}\end{array}$ & $m$ & $\begin{array}{l}m \\
a\end{array}$ & $\begin{array}{l}0 \\
\text { á }\end{array}$ & $\stackrel{n}{i}$ & $m$ \\
\hline 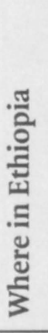 & 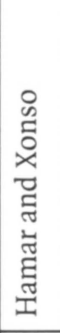 & 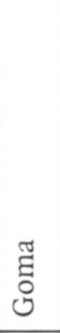 & 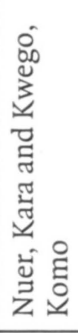 & 总 & 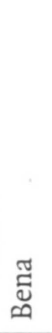 & 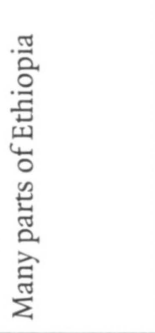 & $\stackrel{\breve{g}}{\stackrel{z}{z}}$ & 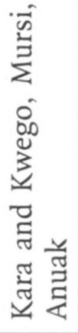 & 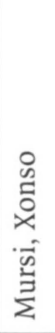 & 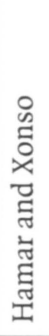 & 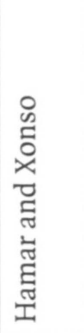 & 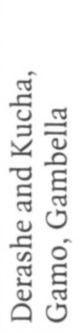 & $\stackrel{\bar{n}}{\stackrel{5}{\Xi}}$ \\
\hline 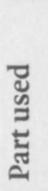 & 岕 & 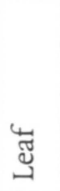 & $\begin{array}{l}0 \\
0 \\
0 \\
0 \\
1 \\
\infty \\
0 \\
\Xi \\
0 \\
0\end{array}$ & 嵌 & 嵌 & 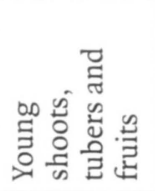 & 鸪 & 莺 & 芯 & 䍖 & 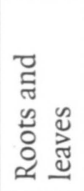 & 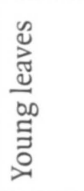 & 㟢 \\
\hline $\begin{array}{l}\text { : } \\
\text { 茔 } \\
\text { I }\end{array}$ & 0 & is & I & 工 & 工 & 0 & I & 0 & is & $\mapsto$ & I & I & I \\
\hline 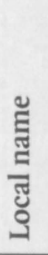 & 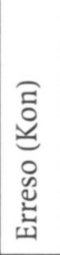 & 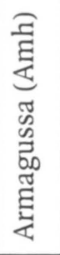 & 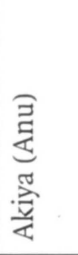 & 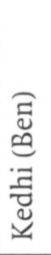 & 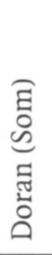 & 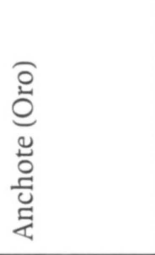 & 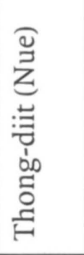 & 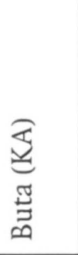 & 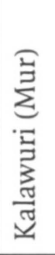 & 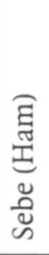 & 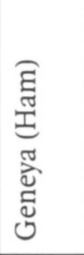 & $\begin{array}{l}\text { (3) } \\
0 \\
3 \\
3 \\
3\end{array}$ & 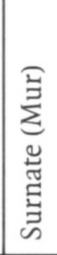 \\
\hline 㙳 & 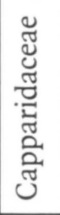 & 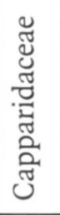 & 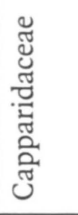 & 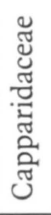 & 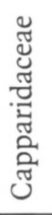 & 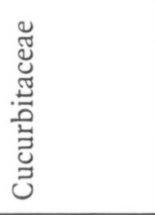 & 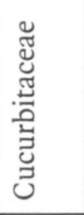 & 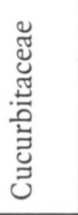 & 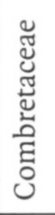 & 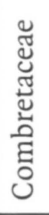 & 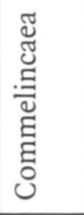 & 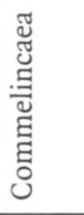 & 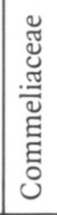 \\
\hline 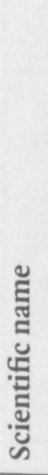 & 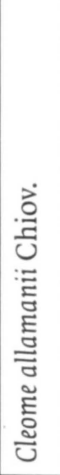 & 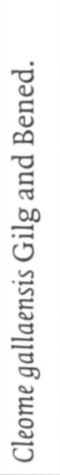 & 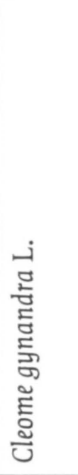 & 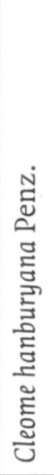 & 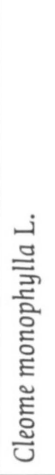 & 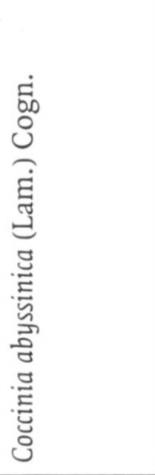 & 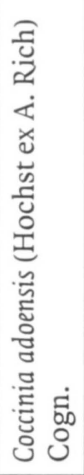 & 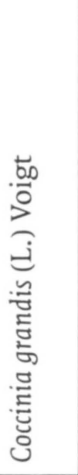 & 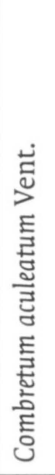 & 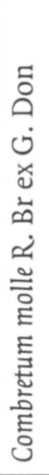 & 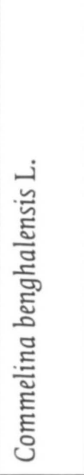 & 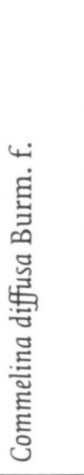 & 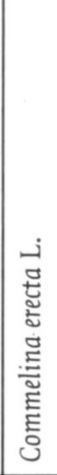 \\
\hline z & N & $n$ & む゙ & น & 으 & ล̊ & $\stackrel{\infty}{\circ}$ & ฉ & 임 & 엄 & న̃ & $\stackrel{\circ}{\circ}$ & ১ \\
\hline
\end{tabular}




\begin{tabular}{|c|c|c|c|c|c|c|c|c|c|c|c|c|c|c|}
\hline 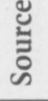 & $a$ & $\stackrel{m}{a}$ & $a$ & $\stackrel{m}{\tilde{n}}$ & $m$ & $\Lambda$ & $m$ & $a$ & $a$ & $a$ & $m$ & $a$ & $a$ & $N$ \\
\hline 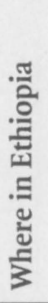 & 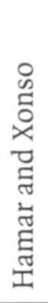 & 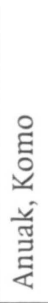 & 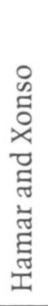 & 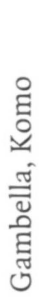 & 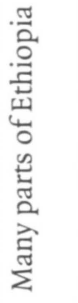 & 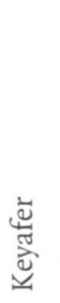 & 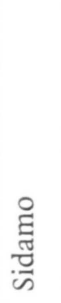 & 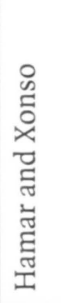 & 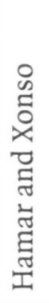 & 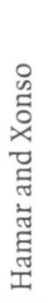 & $\frac{\stackrel{0}{\pi}}{\stackrel{\pi}{\Xi}}$ & 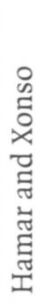 & 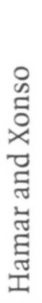 & 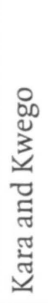 \\
\hline 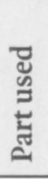 & 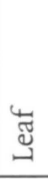 & 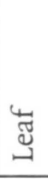 & 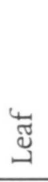 & 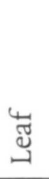 & 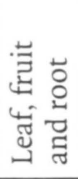 & 壱 & 鸪 & $\begin{array}{l}\ddot{\circ} \\
\stackrel{2}{\alpha}\end{array}$ & 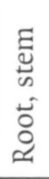 & ঙ্త & 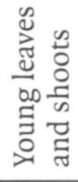 & 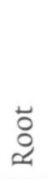 & ¿ః & 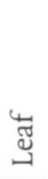 \\
\hline $\begin{array}{l}\text { 节 } \\
\text { 吾 }\end{array}$ & $I$ & I & I & 工 & is & $\mapsto$ & is & $\mapsto$ & $\mapsto$ & $H$ & $\infty$ & $H$ & 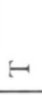 & I \\
\hline 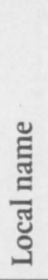 & 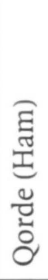 & 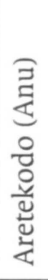 & 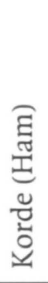 & 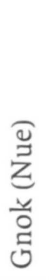 & 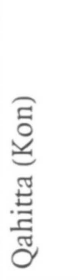 & 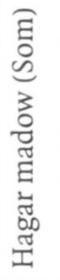 & 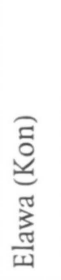 & 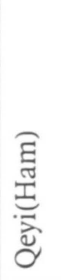 & 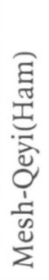 & 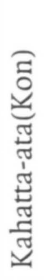 & 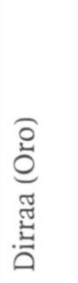 & 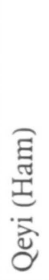 & 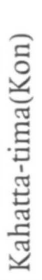 & $\begin{array}{l}\frac{3}{y} \\
\frac{y}{0} \\
\frac{0}{0}\end{array}$ \\
\hline 公 & 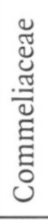 & 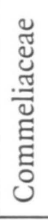 & 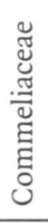 & 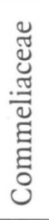 & 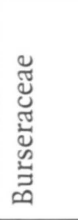 & 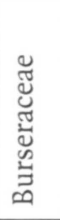 & 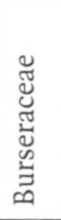 & 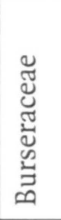 & 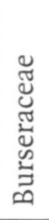 & 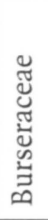 & 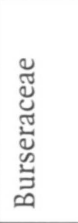 & 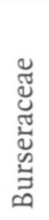 & 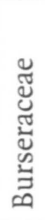 & 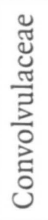 \\
\hline 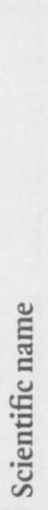 & 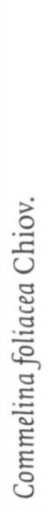 & 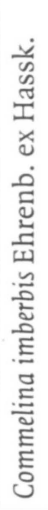 & 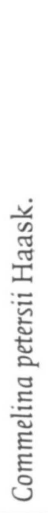 & 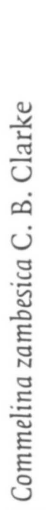 & 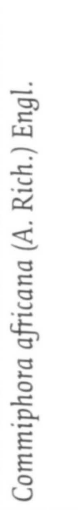 & 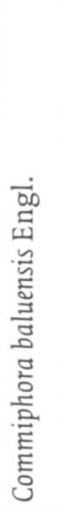 & 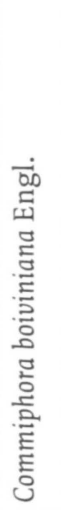 & 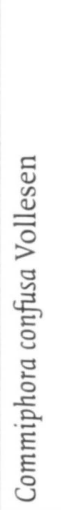 & 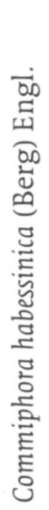 & 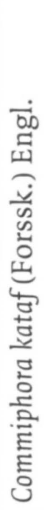 & 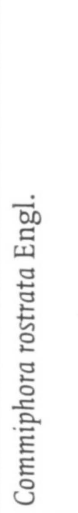 & 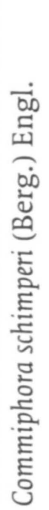 & 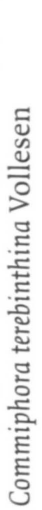 & 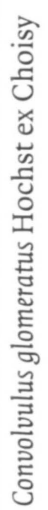 \\
\hline z & 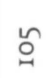 & 엉 & 옹 & $\stackrel{\infty}{\infty}$ & ๖े & $\stackrel{\circ}{\Xi}$ & $\Xi$ & $\stackrel{\Xi}{\Xi}$ & $\cong$ & $\underset{G}{\mathbb{J}}$ & $\cong$ & $\stackrel{\Xi}{\exists}$ & $\Xi$ & $\stackrel{\infty}{\sharp}$ \\
\hline
\end{tabular}




\begin{tabular}{|c|c|c|c|c|c|c|c|c|c|c|c|c|c|}
\hline 苋 & $a$ & $m$ & $\tilde{n}$ & $m$ & $\begin{array}{l}0 \\
i \\
i \\
i\end{array}$ & $\hat{a}$ & $\begin{array}{l}\hat{n} \\
\hat{n}\end{array}$ & $m$ & $\begin{array}{l}n \\
\tilde{0} \\
\dot{m}\end{array}$ & $m$ & - & $\begin{array}{l}m \\
i\end{array}$ & $m$ \\
\hline 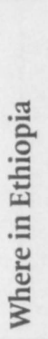 & 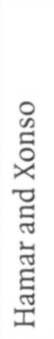 & 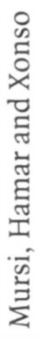 & 光 & 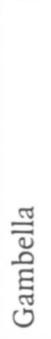 & 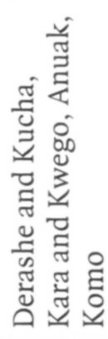 & 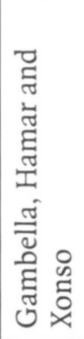 & 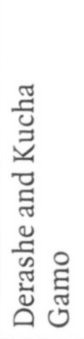 & 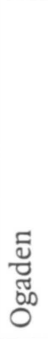 & 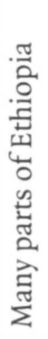 & 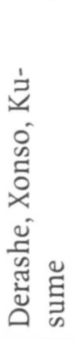 & 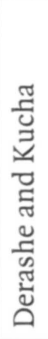 & 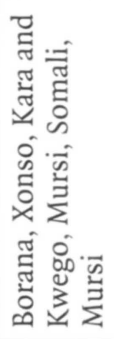 & $\begin{array}{l}\text { ज्ञ } \\
\text { Оू } \\
\text { हु }\end{array}$ \\
\hline 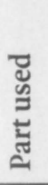 & 㟢 & $\begin{array}{l}\frac{0}{0} \\
\frac{1}{3}\end{array}$ & $\stackrel{\widetilde{J}}{\leftrightarrows}$ & 亗 & 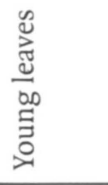 & 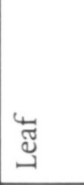 & 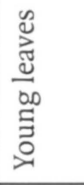 & జ్ల & 莺 & 莺 & 莺 & 叴 & 荘 \\
\hline $\begin{array}{l}\text { : } \\
\text { 荧 }\end{array}$ & 0 & I & I & $I$ & I & I & I & $\infty$ & $r$ & - & is & $r$ & $I$ \\
\hline 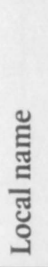 & 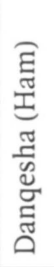 & 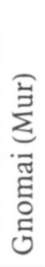 & 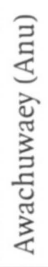 & 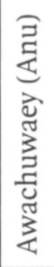 & 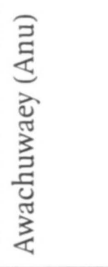 & 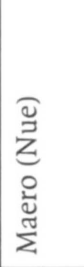 & 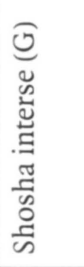 & 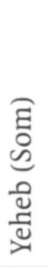 & 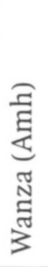 & 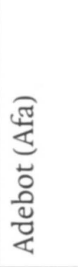 & 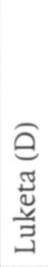 & 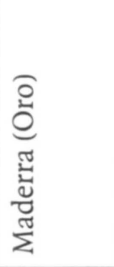 & 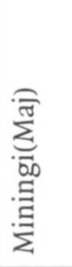 \\
\hline 永 & 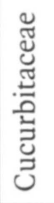 & 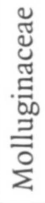 & 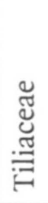 & 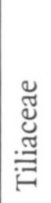 & 芯 & 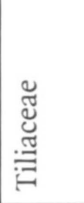 & 芯 & 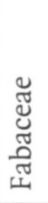 & 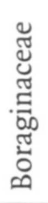 & 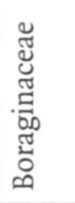 & 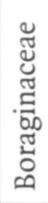 & 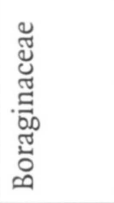 & 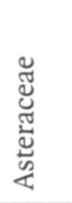 \\
\hline 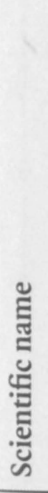 & 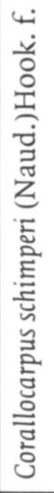 & 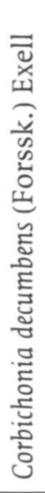 & 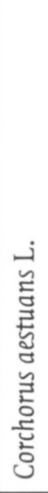 & 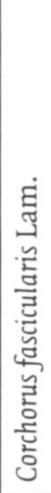 & 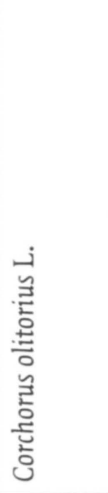 & 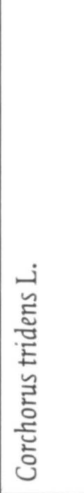 & 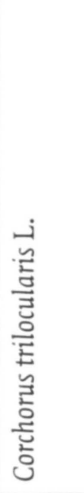 & 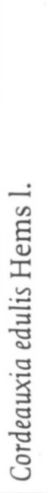 & 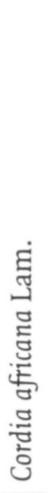 & 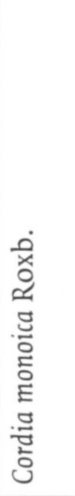 & 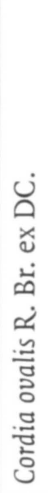 & 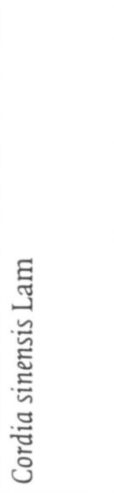 & 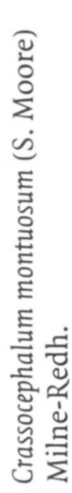 \\
\hline$\stackrel{0}{z}$ & $\stackrel{g}{a}$ & $\tau$ & $\tilde{\Xi}$ & $\mathbb{Z}$ & $\stackrel{n}{\mathfrak{N}}$ & ב্র & 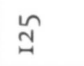 & స్తి & $\widehat{\hat{N}}$ & $\stackrel{\infty}{\underset{N}{N}}$ & త్స & 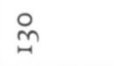 & $\vec{n}$ \\
\hline
\end{tabular}




\begin{tabular}{|c|c|c|c|c|c|c|c|c|c|c|c|c|c|c|}
\hline 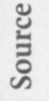 & $\stackrel{n}{-}$ & $\stackrel{n}{-}$ & $a$ & $a$ & $a$ & $\Lambda$ & $a$ & $\Lambda$ & $\Lambda$ & $\stackrel{m}{-1}$ & $\hat{a}$ & $m$ & $a$ & $\checkmark$ \\
\hline 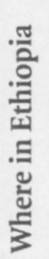 & 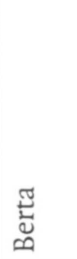 & 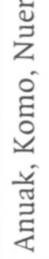 & 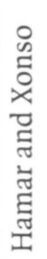 & 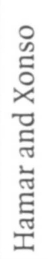 & 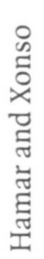 & 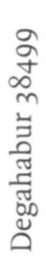 & 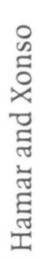 & 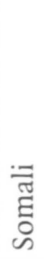 & 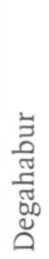 & 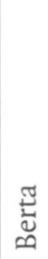 & 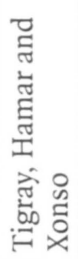 & 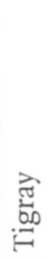 & 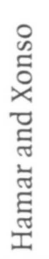 & 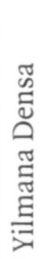 \\
\hline 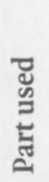 & 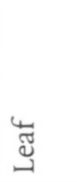 & 壱 & 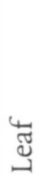 & 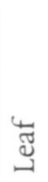 & 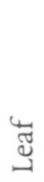 & 光 & 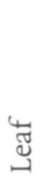 & 壱 & 壱 & 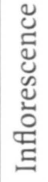 & $\stackrel{0}{\exists}$ & 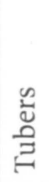 & $\begin{array}{l}\check{0} \\
\stackrel{1}{\circ}\end{array}$ & $\underset{\infty}{\partial}$ \\
\hline 营 & I & $\infty$ & I & I & I & $\cup$ & $\cup$ & en & $\cup$ & $I$ & I & $I$ & $I$ & I \\
\hline 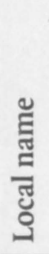 & 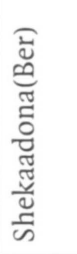 & 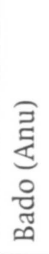 & 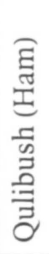 & 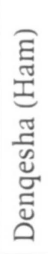 & 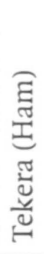 & 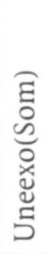 & 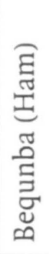 & 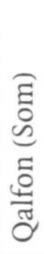 & 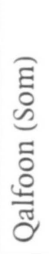 & 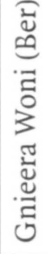 & $\begin{array}{l}\stackrel{000}{\Xi} \\
\stackrel{\Xi}{\Xi} \\
\underset{\Xi}{\Xi}\end{array}$ & 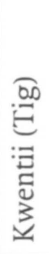 & 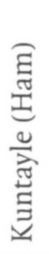 & 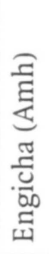 \\
\hline 斏 & 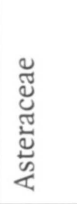 & 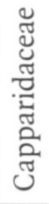 & 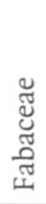 & 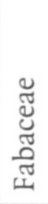 & 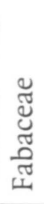 & 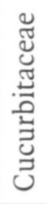 & 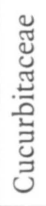 & 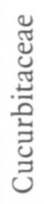 & 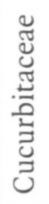 & 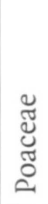 & 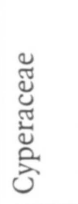 & 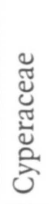 & 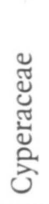 & 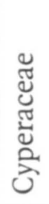 \\
\hline 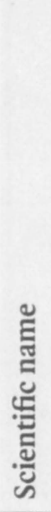 & 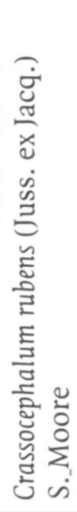 & 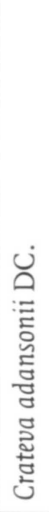 & 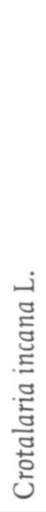 & 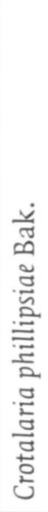 & 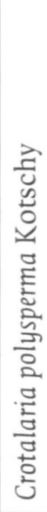 & 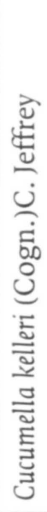 & 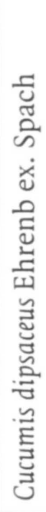 & 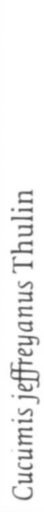 & 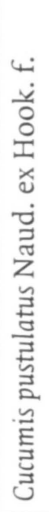 & 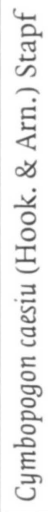 & 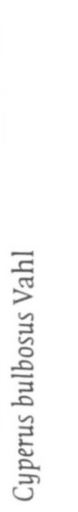 & 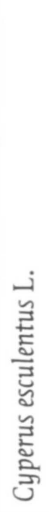 & 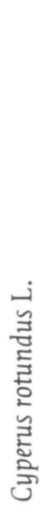 & 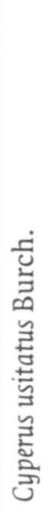 \\
\hline 之 & $\stackrel{N}{n}$ & $\stackrel{n}{n}$ & $\stackrel{+}{\sim}$ & $\tilde{n}$ & $\stackrel{0}{\sim}$ & $\hat{n}$ & $\stackrel{\infty}{m}$ & $\stackrel{\Re}{=}$ & 导 & 莳 & 广 & $\dddot{\sharp}$ & ষ & 先 \\
\hline
\end{tabular}




\begin{tabular}{|c|c|c|c|c|c|c|c|c|c|c|c|c|c|}
\hline 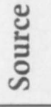 & $N$ & t & $a$ & $\hat{m}$ & $m$ & $\stackrel{m}{\tilde{m}}$ & $\begin{array}{l}\circ \\
\dot{n}\end{array}$ & $\begin{array}{l}\cong \\
i\end{array}$ & $n$ & $m$ & $\begin{array}{l}\hat{a} \\
\hat{n} \\
\hat{i}\end{array}$ & $\stackrel{\circ}{\dot{m}}$ & $\begin{array}{l}i a \\
i m\end{array}$ \\
\hline 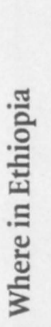 & 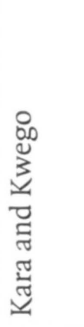 & 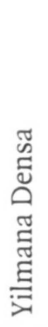 & 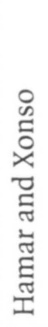 & $\begin{array}{l}0 \\
\stackrel{2}{0} \\
\text { ¿ }\end{array}$ & 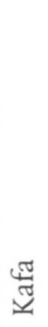 & 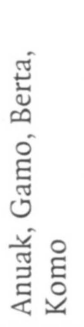 & 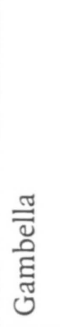 & 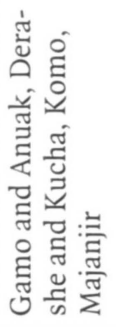 & $\underset{\mathscr{\pi}}{\stackrel{\pi}{\pi}}$ & 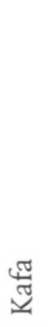 & 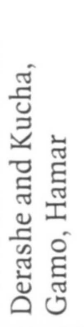 & 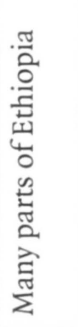 & 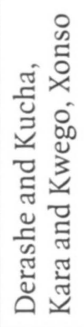 \\
\hline 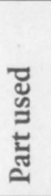 & 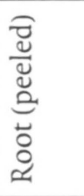 & $\begin{array}{l}\text { تّٓ } \\
\text { Z }\end{array}$ & ¿্ّ & 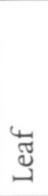 & 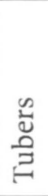 & $\begin{array}{l}\stackrel{2}{\breve{\Xi}} \\
\stackrel{\Xi}{\Xi}\end{array}$ & $\begin{array}{l}\breve{\circ} \\
\stackrel{2}{2}\end{array}$ & $\begin{array}{l}\stackrel{2}{\grave{U}} \\
\stackrel{\Xi}{\Xi}\end{array}$ & $\begin{array}{l}\stackrel{\infty}{\tilde{U}} \\
\Xi \\
\Xi\end{array}$ & ڤั & 莺 & 莺 & 芯 \\
\hline 章 & $I$ & in & $r$ & $I$ & 0 & 0 & $I$ & 0 & 0 & 0 & $\mapsto$ & $r$ & 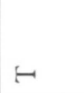 \\
\hline 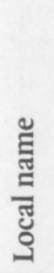 & $\begin{array}{l}\mathbf{d} \\
己 \\
0 \\
0 \\
0 \\
0\end{array}$ & 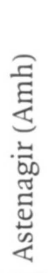 & 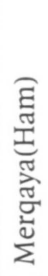 & 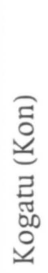 & 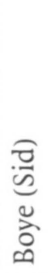 & 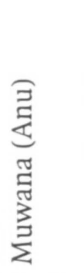 & $\sum_{Z}$ & 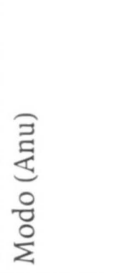 & $\begin{array}{l}\stackrel{\partial}{0} \\
\stackrel{0}{0} \\
\stackrel{0}{\vec{z}}\end{array}$ & 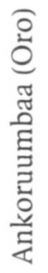 & 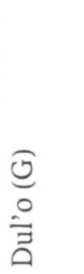 & 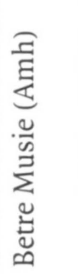 & 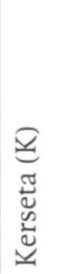 \\
\hline 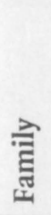 & 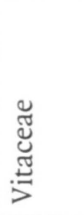 & 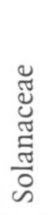 & 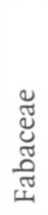 & 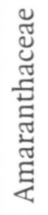 & 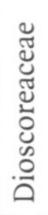 & 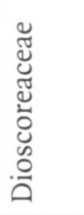 & 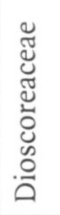 & 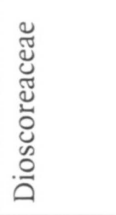 & 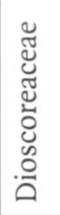 & 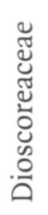 & 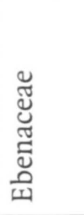 & 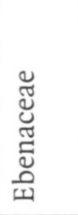 & 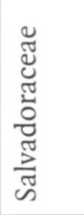 \\
\hline 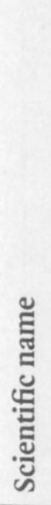 & 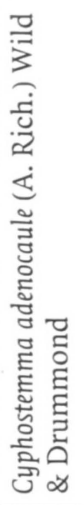 & 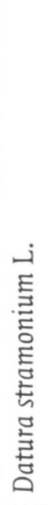 & 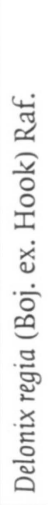 & 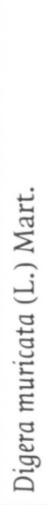 & 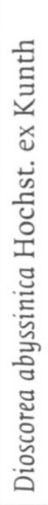 & 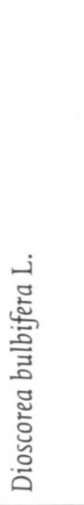 & 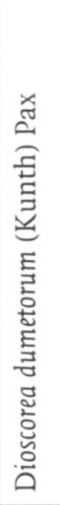 & 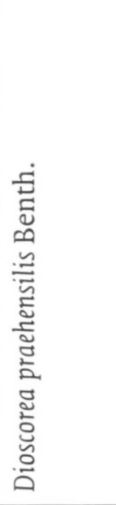 & 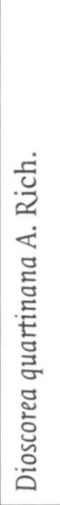 & 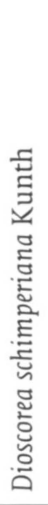 & 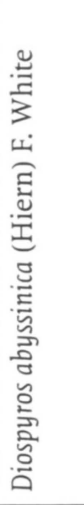 & 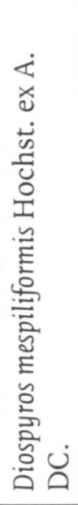 & 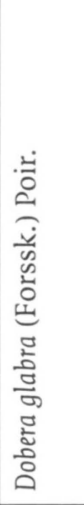 \\
\hline ż & 卞 & 吉 & $\stackrel{\infty}{\text { J }}$ & g & $\stackrel{\sim}{\sim}$ & 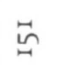 & $\stackrel{N}{\sim}$ & $\stackrel{n}{n}$ & $\stackrel{ \pm}{\leftrightarrows}$ & $\stackrel{n}{n}$ & $\stackrel{\leftrightarrow}{\sim}$ & $\stackrel{\sim}{\sim}$ & $\stackrel{\infty}{\curvearrowleft}$ \\
\hline
\end{tabular}




\begin{tabular}{|c|c|c|c|c|c|c|c|c|c|c|c|c|c|}
\hline $\begin{array}{l}\text { U్ } \\
\text { ¿ }\end{array}$ & $a$ & $m$ & $a$ & $m$ & $m$ & $\stackrel{m}{i}$ & $n$ & $\tilde{n}$ & $r$ & $a$ & $a$ & $a$ & ナ \\
\hline 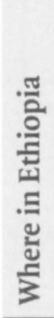 & 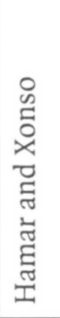 & 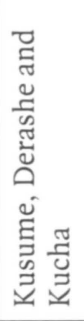 & 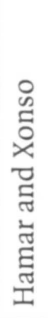 & 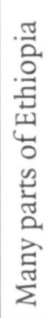 & 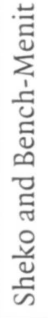 & 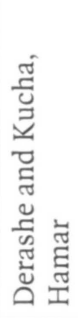 & 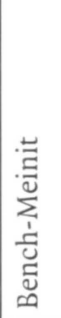 & $\begin{array}{l}: \frac{\pi}{\bar{E}} \\
\frac{\pi}{\pi} \\
\sum\end{array}$ & 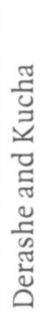 & 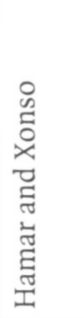 & 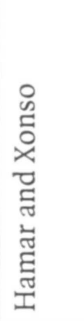 & 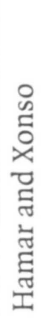 & $\begin{array}{l}\mathbb{Z} \\
\text { ㅁ }\end{array}$ \\
\hline 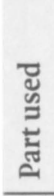 & 总 & 壱 & ¿ّ & 蠧 & 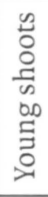 & 节 & 总 & 节 & 売 & 芯 & 芯 & జ్ల & 总 \\
\hline 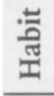 & is & H & I & $\infty$ & is & $H$ & $H$ & $H$ & en & I & 工 & I & $H$ \\
\hline 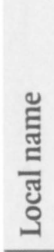 & 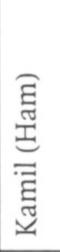 & 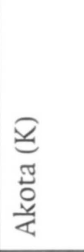 & 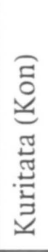 & 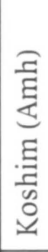 & 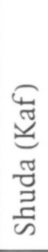 & 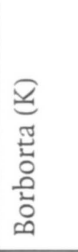 & 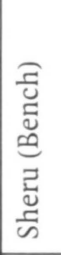 & 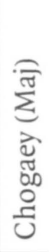 & 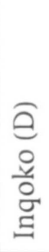 & 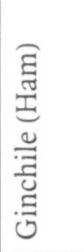 & 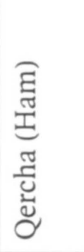 & 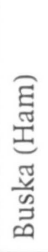 & 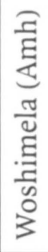 \\
\hline 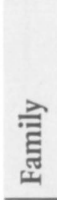 & 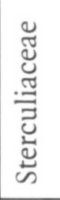 & 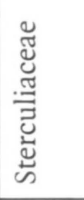 & 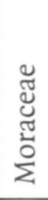 & 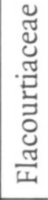 & 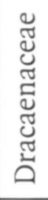 & 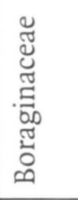 & 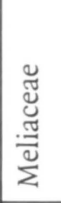 & 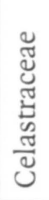 & 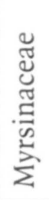 & 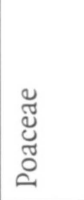 & 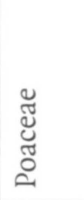 & 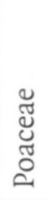 & 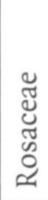 \\
\hline 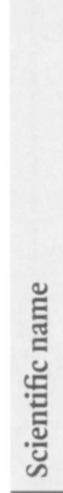 & 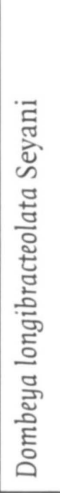 & 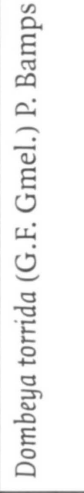 & 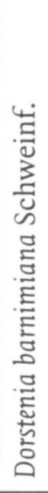 & 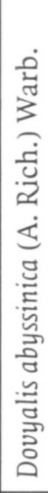 & 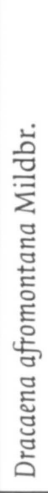 & 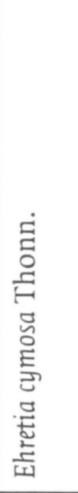 & 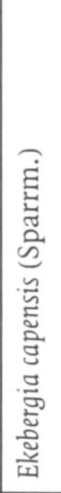 & 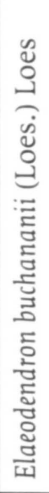 & 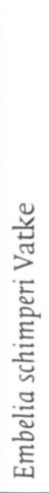 & 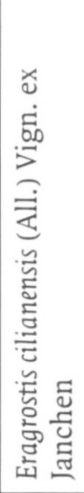 & 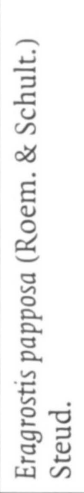 & 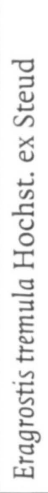 & 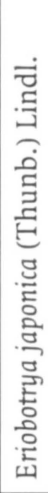 \\
\hline$\stackrel{\circ}{z}$ & 怘 & $\stackrel{\circ}{\circ}$ & $\vec{\sigma}$ & ֻِ & $\stackrel{m}{\underline{\sigma}}$ & త్ర & $\stackrel{\text { ڤn }}{\circ}$ & : & 음 & 央 & o్రి & $\stackrel{0}{\stackrel{ }{~}}$ & $\overrightarrow{\widehat{N}}$ \\
\hline
\end{tabular}




\begin{tabular}{|c|c|c|c|c|c|c|c|c|c|c|c|c|c|}
\hline 芯 & $\begin{array}{l}\stackrel{\circ}{m} \\
\dot{m}\end{array}$ & $m$ & $n$ & $\exists$ & $a$ & $\stackrel{n}{\sim}$ & $\begin{array}{l}a \\
\hat{n} \\
i\end{array}$ & $n$ & + & $a$ & $m$ & m & $m$ \\
\hline 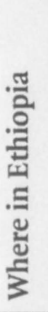 & 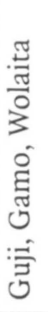 & 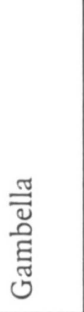 & 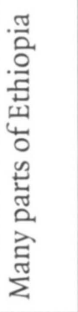 & 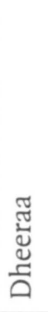 & 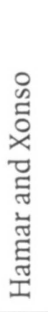 & 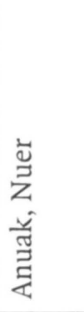 & 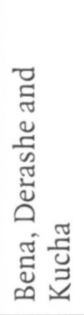 & 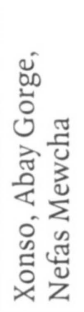 & $\begin{array}{l}\underset{\widetilde{J}}{\mathbb{Z}} \\
\text { 党 }\end{array}$ & 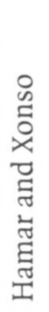 & 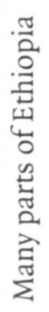 & 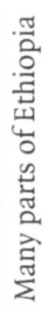 & 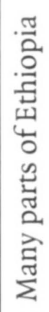 \\
\hline 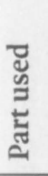 & $\begin{array}{l}\breve{\circ} \\
\stackrel{2}{ }\end{array}$ & 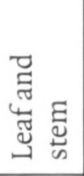 & 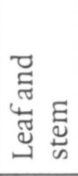 & ¿े & 壱 & 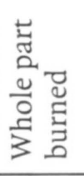 & 莺 & 坣 & 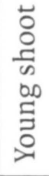 & 莺 & 壱 & 寻 & 羙 \\
\hline $\begin{array}{l}\text { 苟 } \\
\text { 荧 }\end{array}$ & I & I & I & 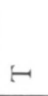 & is & I & $\mapsto$ & $\mapsto$ & I & $\mapsto$ & $\mapsto$ & $\mapsto$ & $H$ \\
\hline 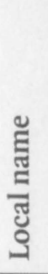 & 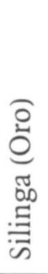 & 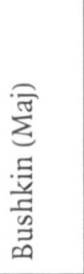 & 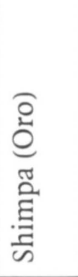 & 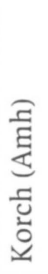 & 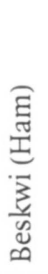 & 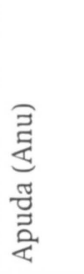 & 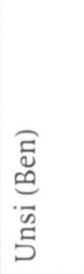 & 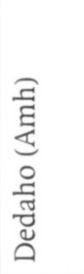 & 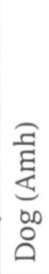 & 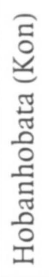 & $\begin{array}{l}\text { 恶 } \\
\text { 总 } \\
\text { 品 }\end{array}$ & 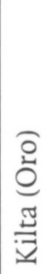 & 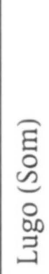 \\
\hline 永 & 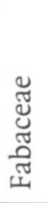 & 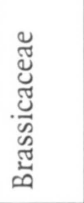 & 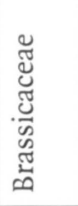 & 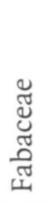 & 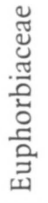 & 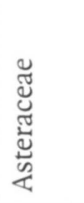 & 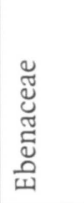 & 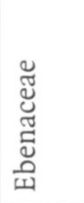 & 芯 & 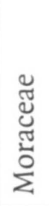 & 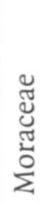 & 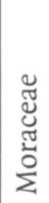 & 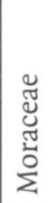 \\
\hline 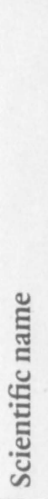 & 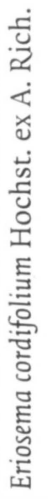 & 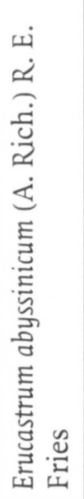 & 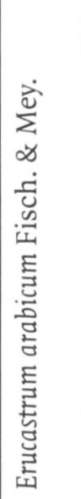 & 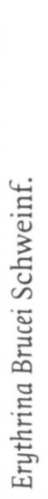 & 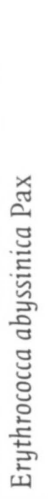 & 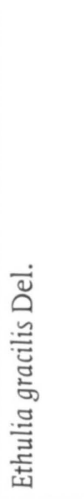 & 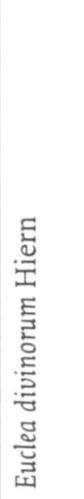 & 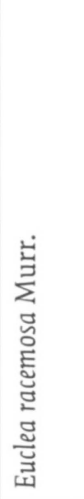 & 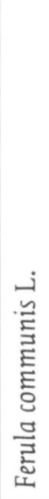 & 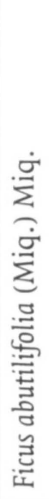 & 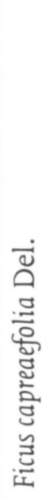 & 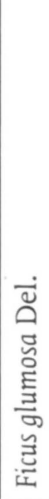 & 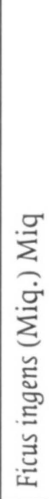 \\
\hline$\stackrel{0}{z}$ & $\stackrel{N}{\stackrel{N}{\Lambda}}$ & 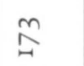 & $\stackrel{+}{\stackrel{\Delta}{\sigma}}$ & $\stackrel{n}{\bumpeq}$ & 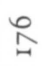 & $\stackrel{\curvearrowright}{A}$ & $\stackrel{\infty}{\stackrel{\infty}{\Lambda}}$ & $\stackrel{\Omega}{\curvearrowright}$ & @ & 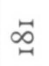 & న్ర & 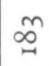 & क् \\
\hline
\end{tabular}




\begin{tabular}{|c|c|c|c|c|c|c|c|c|c|c|c|c|c|}
\hline 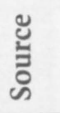 & $m$ & $m$ & $m$ & $a$ & $\hat{m}$ & $\stackrel{i m}{i m}$ & $\begin{array}{l}\dot{0} \\
\dot{a}\end{array}$ & $m$ & $\stackrel{0}{i}$ & $n$ & - & $\begin{array}{l}O \\
\dot{m} \\
i\end{array}$ & $a$ \\
\hline 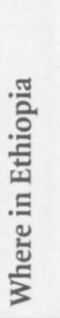 & 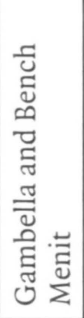 & 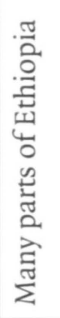 & 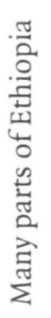 & 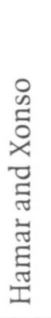 & 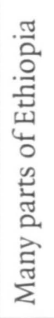 & 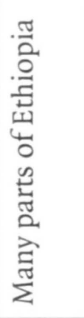 & 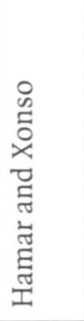 & 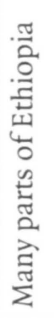 & 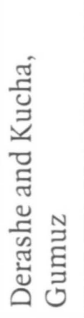 & 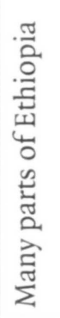 & 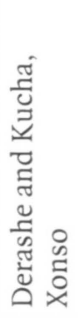 & 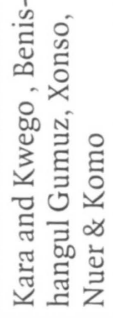 & 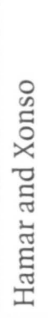 \\
\hline 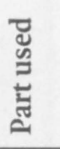 & 壭 & 䓌 & 売 & 总 & 总 & 军 & 点 & 莺 & 莺 & 鸹 & 芯 & 莺 & 㤩 \\
\hline $\begin{array}{l}\text { 竞 } \\
\text { 吾 }\end{array}$ & 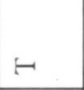 & 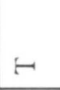 & 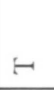 & $r$ & $H$ & $H$ & $H$ & $r$ & $H$ & $H$ & is & 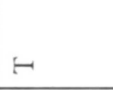 & is \\
\hline 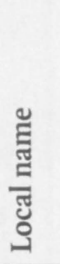 & 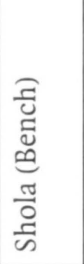 & 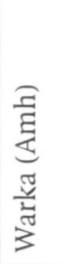 & 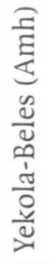 & 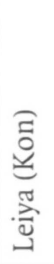 & 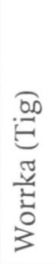 & $\begin{array}{l}30 \\
3 \\
\frac{\pi}{0} \\
3\end{array}$ & 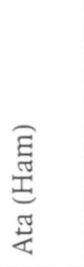 & 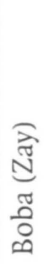 & 突 & 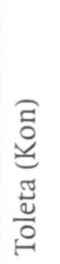 & 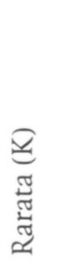 & 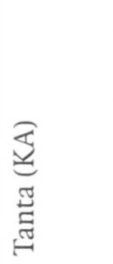 & 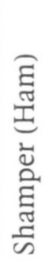 \\
\hline 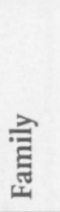 & 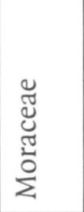 & 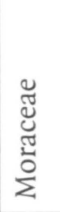 & 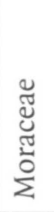 & 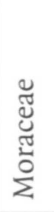 & 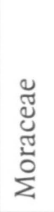 & 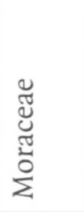 & 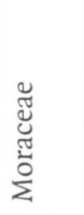 & 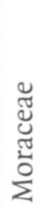 & 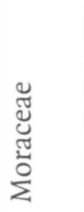 & 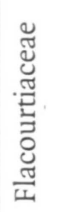 & 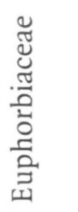 & 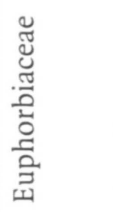 & 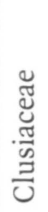 \\
\hline 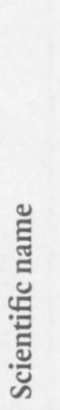 & 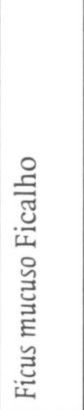 & 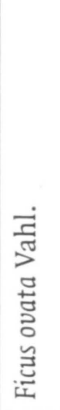 & 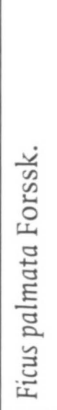 & 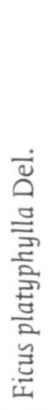 & 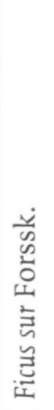 & 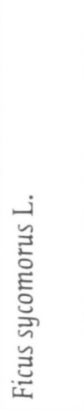 & 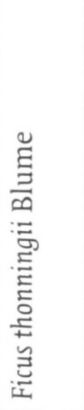 & 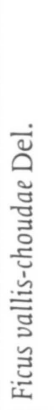 & 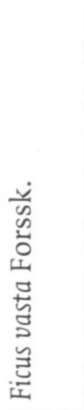 & 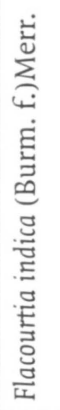 & 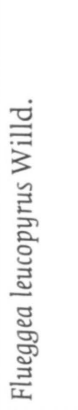 & 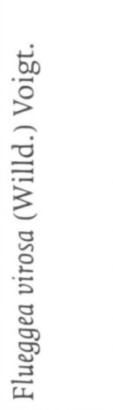 & 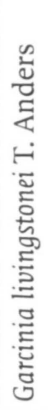 \\
\hline$\grave{z}$ & $\stackrel{n}{\infty}$ & $\infty$ & 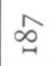 & $\underset{\sim}{\infty}$ & $\infty$ & ஓ & ลี & 9 & $\stackrel{n}{a}$ & ず & 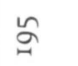 & $\stackrel{9}{9}$ & $\hat{\Omega}$ \\
\hline
\end{tabular}




\begin{tabular}{|c|c|c|c|c|c|c|c|c|c|c|c|c|c|}
\hline $\begin{array}{l}\text { 巳̆ } \\
\text { ¿े }\end{array}$ & $m$ & $\wedge$ & 음 & $m$ & $m$ & $a$ & $\begin{array}{l}n \\
i \\
-\end{array}$ & $m$ & $m$ & $\hat{a}$ & $N$ & $a$ & $\begin{array}{l}\cong \\
\tilde{0} \\
\dot{m} \\
\dot{n}\end{array}$ \\
\hline 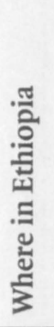 & 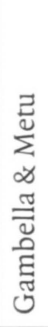 & 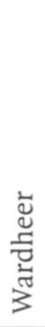 & 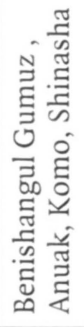 & 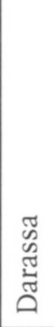 & 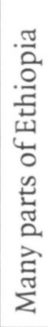 & $\begin{array}{l}0 \\
\text { D } \\
0 \\
0\end{array}$ & 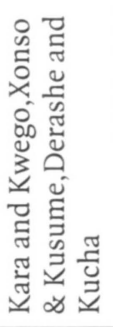 & 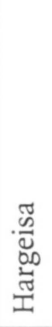 & 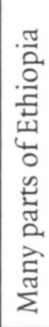 & 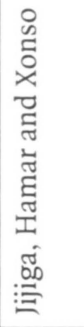 & 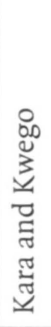 & 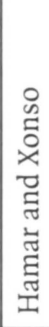 & 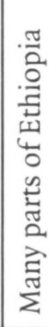 \\
\hline 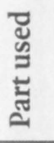 & 壱 & 叴 & 㤩 & 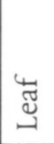 & 寻 & 常 & 壱 & 莺 & 莺 & 总 & 总 & 鸪 & 壱 \\
\hline 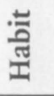 & $t$ & in & is & $I$ & $t$ & $t$ & 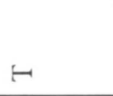 & es & $H$ & in & is & is & $H$ \\
\hline 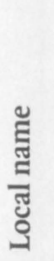 & 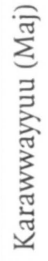 & 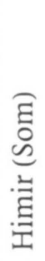 & 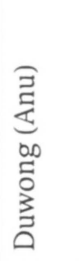 & 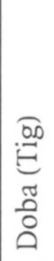 & 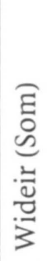 & 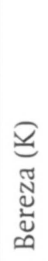 & 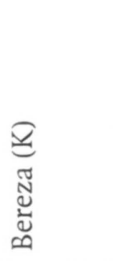 & 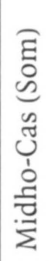 & 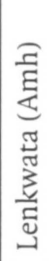 & 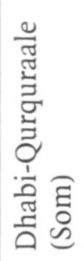 & 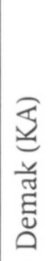 & 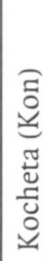 & 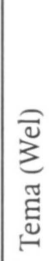 \\
\hline 瓿 & 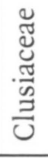 & 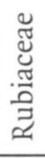 & 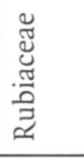 & 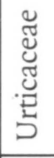 & 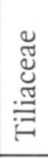 & 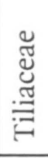 & 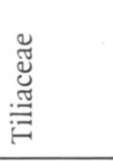 & 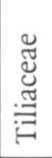 & 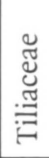 & 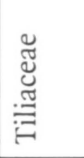 & 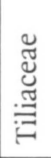 & 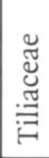 & 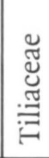 \\
\hline 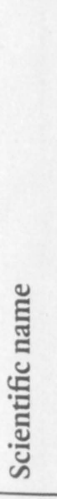 & 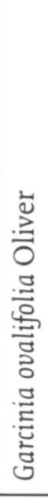 & 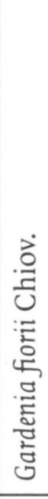 & 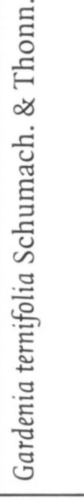 & 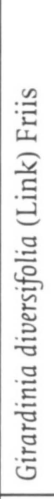 & 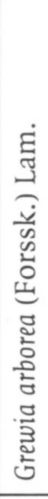 & 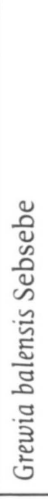 & 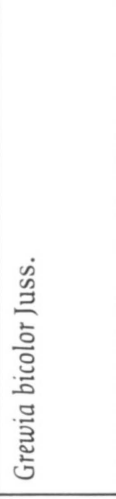 & 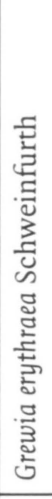 & 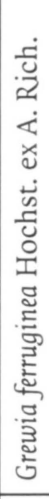 & 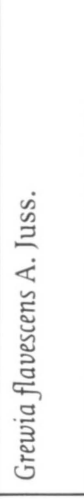 & 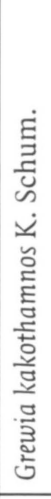 & 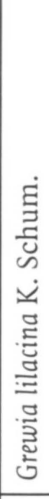 & 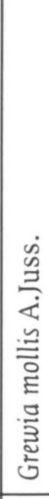 \\
\hline$z^{\circ}$ & $\stackrel{\infty}{2}$ & 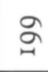 & 유 & กิ & กิ & 우 & गे & กิ & กั & 仓े & 离 & ᄋ్ & 은 \\
\hline
\end{tabular}


E. LULEKAL, Z. ASFAW, E. KELBESSA, P. VAN DAMME

\begin{tabular}{|c|c|c|c|c|c|c|c|c|c|c|c|c|c|}
\hline 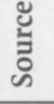 & - & $m$ & $\begin{array}{l}\vec{f} \\
\dot{f}\end{array}$ & $m$ & $\begin{array}{l}\hat{a} \\
\hat{n}\end{array}$ & $m$ & $N$ & $\approx$ & $\cong$ & $\hat{n}$ & $\hat{n}$ & $n$ & $\stackrel{n}{n}$ \\
\hline 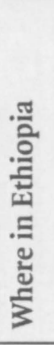 & 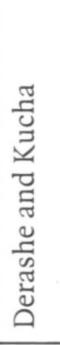 & 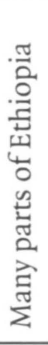 & 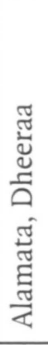 & 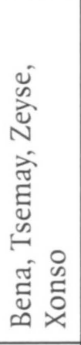 & 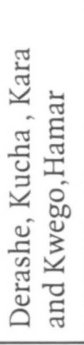 & 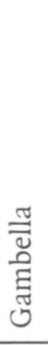 & 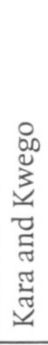 & 莺 & 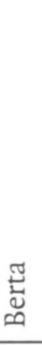 & 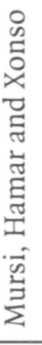 & 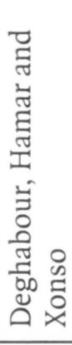 & $\begin{array}{l}\text { 咅 } \\
\text { हี } \\
\text { हुँ }\end{array}$ & 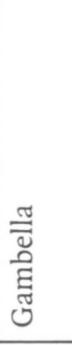 \\
\hline 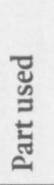 & 峤 & 莺 & 売 & 莺 & 龸 & 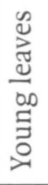 & 壹 & త్త & 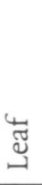 & 壱 & 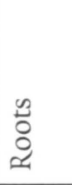 & $\begin{array}{l}\frac{7}{3} \\
\frac{3}{3} \\
\frac{0}{0} \\
\frac{1}{3} \\
\frac{1}{3}\end{array}$ & 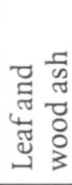 \\
\hline $\begin{array}{l}\text { 音 } \\
\text { 爫 }\end{array}$ & is & is & 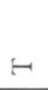 & 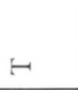 & is & I & $H$ & $I$ & I & $\infty$ & I & I & is \\
\hline 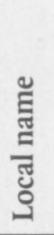 & 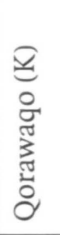 & 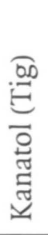 & 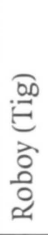 & 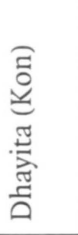 & 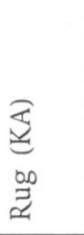 & 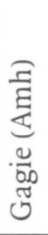 & 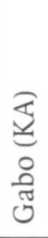 & 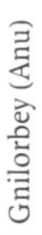 & 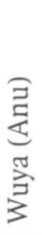 & 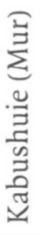 & 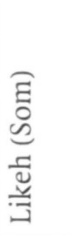 & 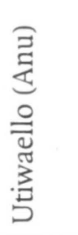 & 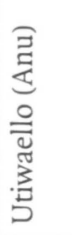 \\
\hline 层 & 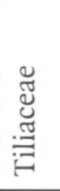 & $\begin{array}{l}\stackrel{\mathscr{J}}{\leftrightarrows} \\
\stackrel{\mathscr{J}}{\Xi}\end{array}$ & 䒿 & 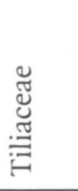 & $\stackrel{\stackrel{\mathscr{J}}{\leftrightarrows}}{\stackrel{\mathscr{J}}{\Xi}}$ & 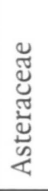 & 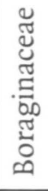 & 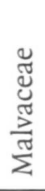 & 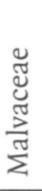 & 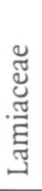 & 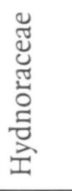 & 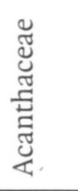 & 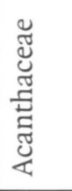 \\
\hline 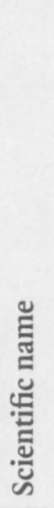 & 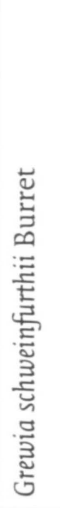 & 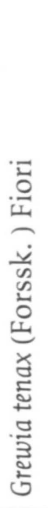 & 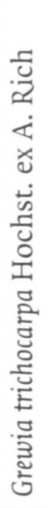 & 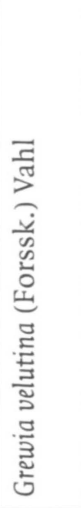 & 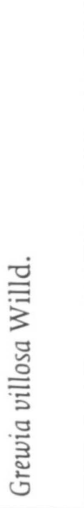 & 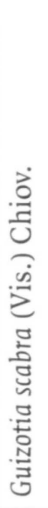 & 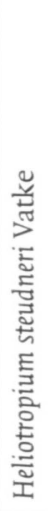 & 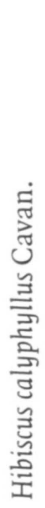 & 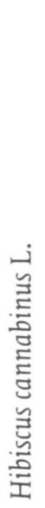 & 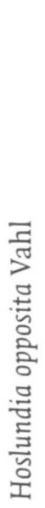 & 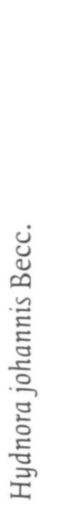 & 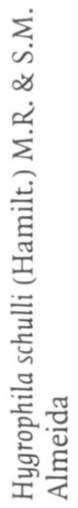 & 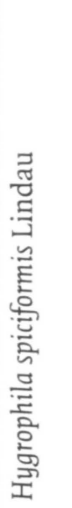 \\
\hline$\stackrel{2}{z}$ & $\overrightarrow{\text { ก }}$ & $\underset{N}{N}$ & $\stackrel{m}{N}$ & ה & $\tilde{N}$ & $\overrightarrow{\mathrm{N}}$ & $\hat{\mathrm{N}}$ & $\vec{N}$ & $\overline{\text { ง }}$ & ปี & $\overrightarrow{\mathrm{N}}$ & N & $\tilde{\mathfrak{N}}$ \\
\hline
\end{tabular}




\begin{tabular}{|c|c|c|c|c|c|c|c|c|c|c|c|c|c|}
\hline 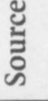 & $m$ & $\hat{m}$ & $n$ & $m$ & $\begin{array}{l}\stackrel{\circ}{m} \\
\dot{m}\end{array}$ & $a$ & $\hat{i}$ & $N$ & $a$ & $a$ & $a$ & $\begin{array}{l}m \\
a \\
a\end{array}$ & $m$ \\
\hline 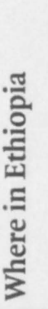 & $\stackrel{\vec{n}}{\stackrel{3}{\Sigma}}$ & 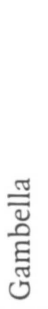 & $\sum_{\Sigma}^{\vec{U}}$ & 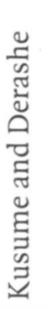 & 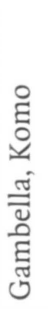 & 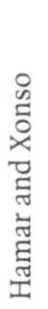 & 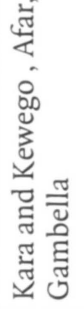 & 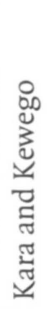 & 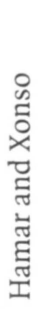 & 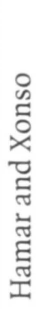 & 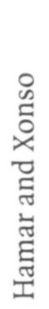 & 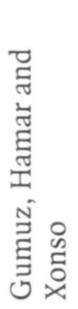 & 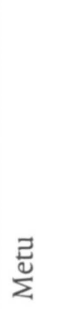 \\
\hline 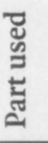 & 鸪 & 毫 & 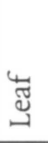 & 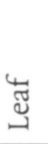 & 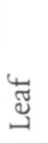 & 叴 & 苂 & $\underset{\mathbb{J}}{\mathbb{J}}$ & 亗 & 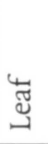 & 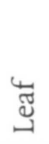 & 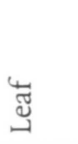 & 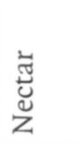 \\
\hline 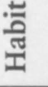 & $H$ & $-H$ & I & I & I & I & is & in & in & I & $I$ & $I$ & in \\
\hline 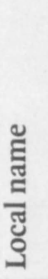 & 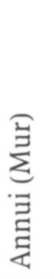 & 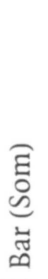 & 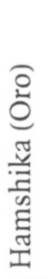 & 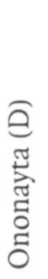 & 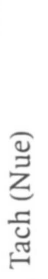 & 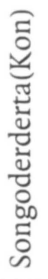 & 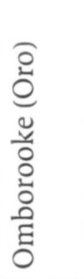 & $\begin{array}{l}\tilde{\Xi} \\
0 \\
\frac{0}{0} \\
\frac{0}{0} \\
0\end{array}$ & 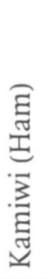 & 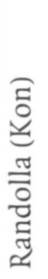 & 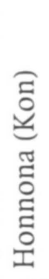 & 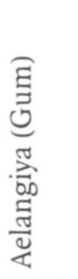 & 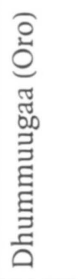 \\
\hline 胥 & 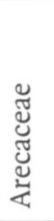 & 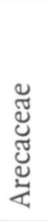 & 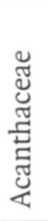 & 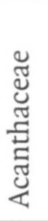 & 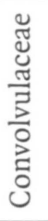 & 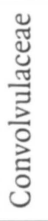 & 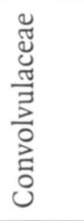 & 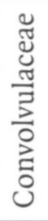 & 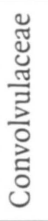 & 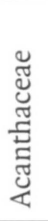 & 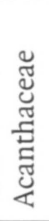 & 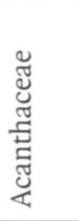 & 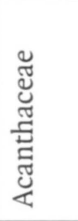 \\
\hline 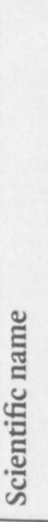 & 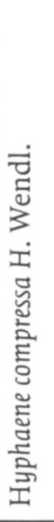 & 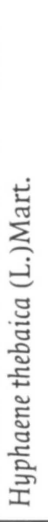 & 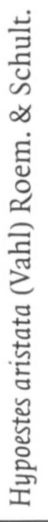 & 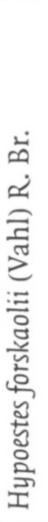 & 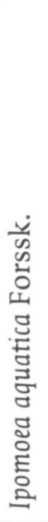 & 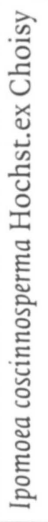 & 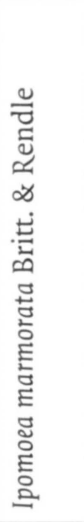 & 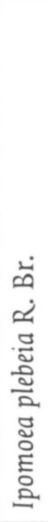 & 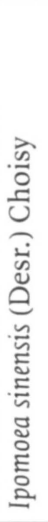 & 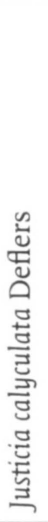 & 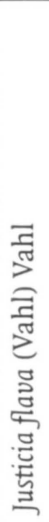 & 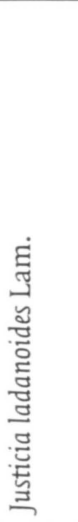 & 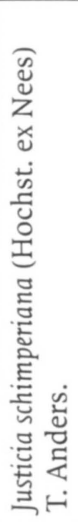 \\
\hline ż & $\underset{\text { N }}{ }$ & $\stackrel{n}{N}$ & ָָ & $\hat{\mathrm{N}}$ & $\underset{\sim}{\infty}$ & స్ & 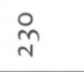 & $\vec{n}$ & $\stackrel{\sim}{\sim}$ & $\stackrel{n}{\sim}$ & $\underset{N}{\sim}$ & $\stackrel{n}{\sim}$ & 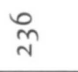 \\
\hline
\end{tabular}


E. LULEKAL, Z. ASFAW, E. KELBESSA, P. VAN DAMME

\begin{tabular}{|c|c|c|c|c|c|c|c|c|c|c|c|c|c|}
\hline 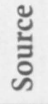 & $\hat{i}$ & $a$ & $\hat{m}$ & $m$ & $\stackrel{m}{m}$ & $H$ & $\hat{m}$ & $m$ & $m$ & $m$ & $m$ & $\cong$ & 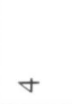 \\
\hline 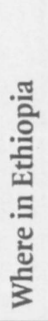 & 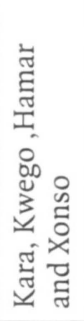 & 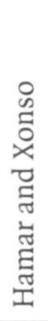 & 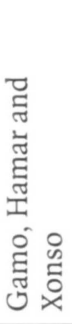 & 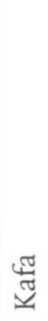 & 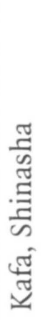 & 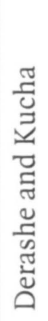 & 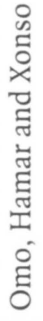 & 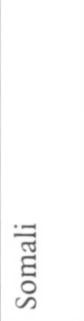 & 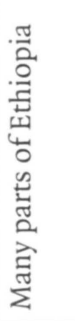 & 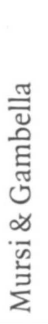 & 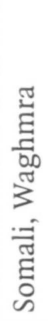 & 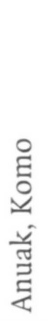 & 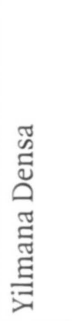 \\
\hline 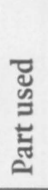 & త్త & 亗 & 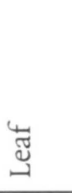 & 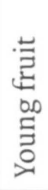 & 莺 & 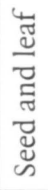 & 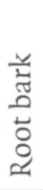 & 苞 & 壳 & 䔍 & $\begin{array}{l}\vec{\circ} \\
\stackrel{2}{\circ}\end{array}$ & 莺 & 茎 \\
\hline 泀 & $u$ & $u$ & $u$ & 0 & 0 & in & $r$ & $r$ & 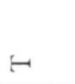 & $\leftarrow$ & in & $H$ & in \\
\hline 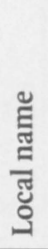 & 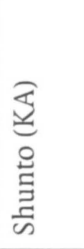 & 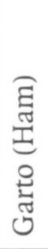 & 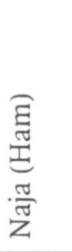 & 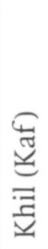 & 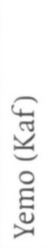 & 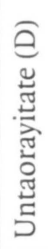 & 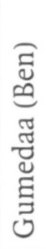 & 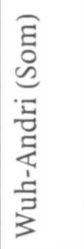 & 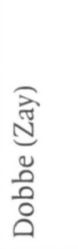 & 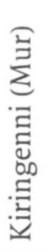 & 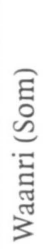 & $\begin{array}{l}\text { 恶 } \\
\text { 恶 }\end{array}$ & 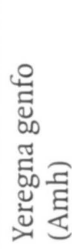 \\
\hline 﨎 & 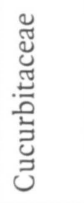 & 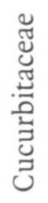 & 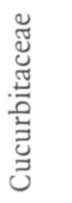 & 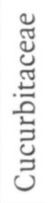 & 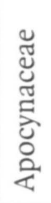 & 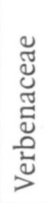 & 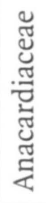 & 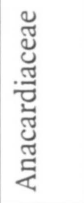 & 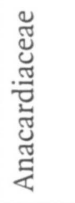 & 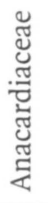 & 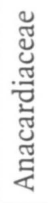 & 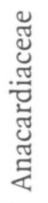 & 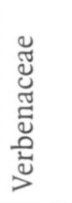 \\
\hline 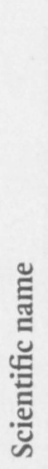 & 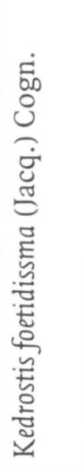 & 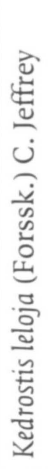 & 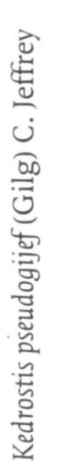 & 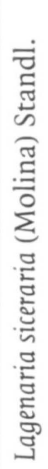 & 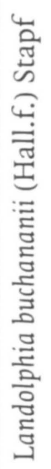 & 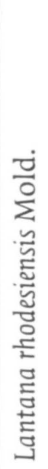 & 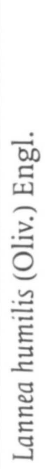 & 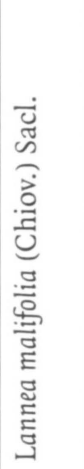 & 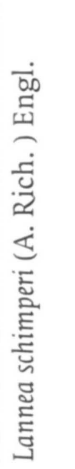 & 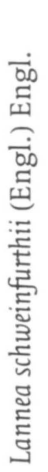 & 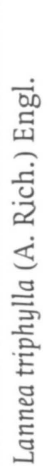 & 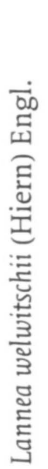 & 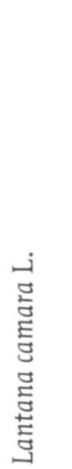 \\
\hline ż & $\hat{n}$ & $\stackrel{\infty}{N}$ & $\overrightarrow{\tilde{N}}$ & गे & त & + & 4े & 守 & $\frac{n}{\mathfrak{q}}$ & لे & ปे & $\stackrel{\infty}{+}$ & 守 \\
\hline
\end{tabular}




\begin{tabular}{|c|c|c|c|c|c|c|c|c|c|c|c|c|c|}
\hline 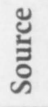 & $m$ & $m$ & $\begin{array}{l}0 \\
\dot{m}\end{array}$ & $\stackrel{n}{\sim}$ & $N$ & $\tilde{n}$ & $\stackrel{m}{i}$ & 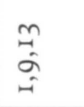 & $a$ & $\tilde{n}$ & 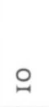 & $N$ & $\hat{m}$ \\
\hline 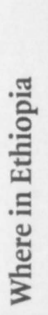 & 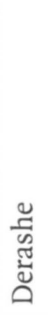 & 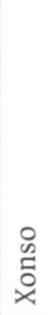 & 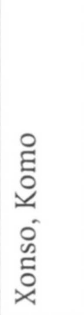 & 莺 & 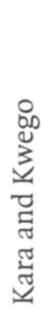 & 莺 & 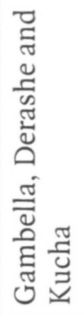 & 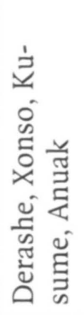 & 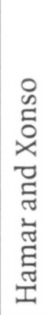 & 弟 & : & 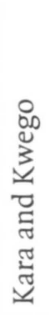 & 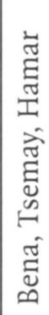 \\
\hline 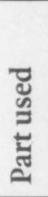 & త్త & 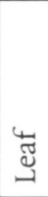 & 荘 & $\begin{array}{l}\text { đัँّ } \\
\text { Zू }\end{array}$ & 䔍 & 吉 & 㤩 & 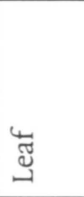 & 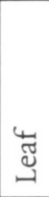 & $\begin{array}{l}\frac{\pi}{4} \\
\frac{0}{4} \\
\frac{0}{0} \\
\frac{\pi}{3}\end{array}$ & 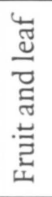 & 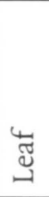 & త్ \\
\hline 言 & in & $I$ & $I$ & $I$ & $\mapsto$ & - & 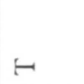 & $u$ & in & $I$ & $u$ & $H$ & in \\
\hline 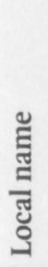 & 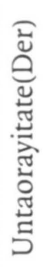 & 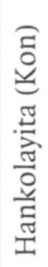 & 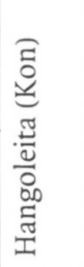 & 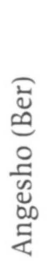 & 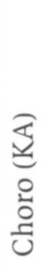 & 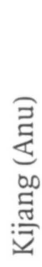 & 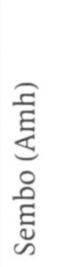 & 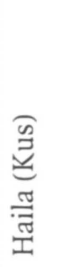 & 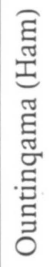 & 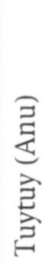 & 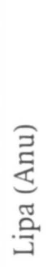 & 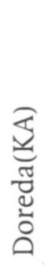 & 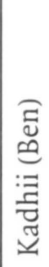 \\
\hline 预 & 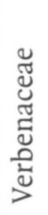 & 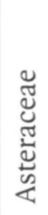 & 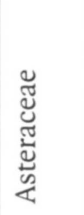 & 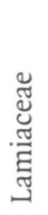 & 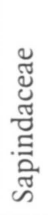 & 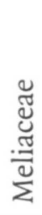 & 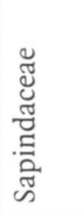 & 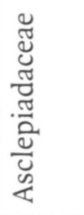 & 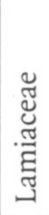 & 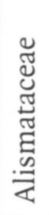 & 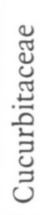 & 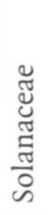 & 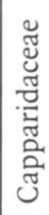 \\
\hline 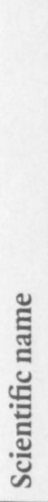 & 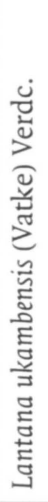 & 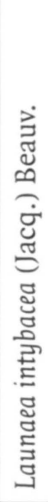 & 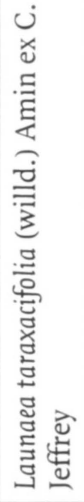 & 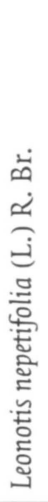 & 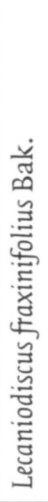 & 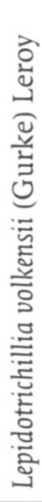 & 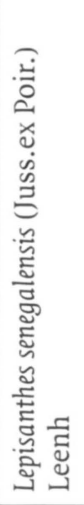 & 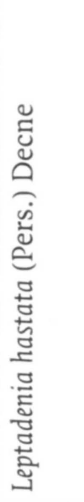 & 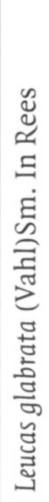 & 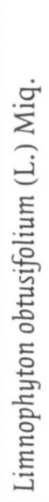 & 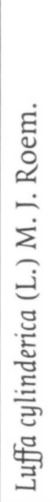 & 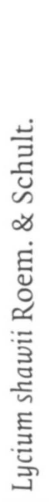 & 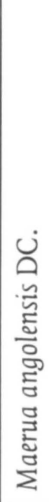 \\
\hline$\stackrel{2}{z}$ & $\stackrel{\circ}{i n}$ & กี & ஸี & $\stackrel{n}{n}$ & $\stackrel{\sim}{\sim}$ & $\sqrt{4}$ & $\stackrel{\circ}{\stackrel{\sim}{N}}$ & $\stackrel{\hat{n}}{n}$ & $\stackrel{\sim}{\sim}$ & 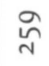 & $\stackrel{\text { : }}{\circ}$ & $\vec{\sim}$ & $\mathscr{N}_{\text {N }}$ \\
\hline
\end{tabular}




\begin{tabular}{|c|c|c|c|c|c|c|c|c|c|c|c|c|c|}
\hline $\begin{array}{l}\text { Uू } \\
\text { ¿ू }\end{array}$ & in & i & $\stackrel{n}{\sim}$ & 임 & $\begin{array}{l}m \\
i\end{array}$ & $\begin{array}{l}\dot{a} \\
\dot{m} \\
\dot{0}\end{array}$ & + & $\stackrel{m}{\tilde{f}}$ & $a$ & $\begin{array}{l}a \\
\dot{n} \\
\hat{i}\end{array}$ & + & $\cong$ & $\stackrel{n}{i}$ \\
\hline 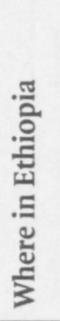 & 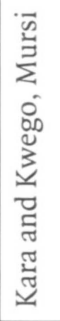 & 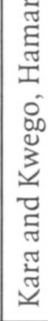 & 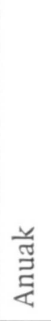 & : & 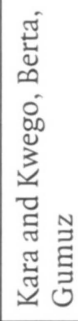 & 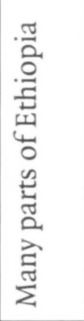 & $\frac{\text { J }}{\frac{\pi}{\mathscr{J}}}$ & 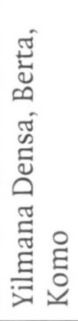 & 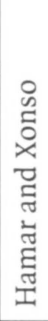 & 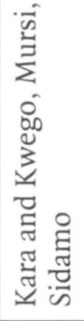 & $\frac{\pi}{\frac{\pi}{\mathscr{V}}}$ & 前 & 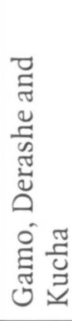 \\
\hline 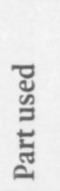 & 岃 & 鸹 & 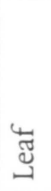 & 壱 & 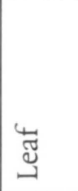 & 㕦 & 莺 & 売 & 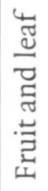 & $\stackrel{\breve{J ~}}{\widetilde{J}}$ & 㕦 & 鸪 & 当 \\
\hline$\frac{\text { 竞 }}{\text { 茥 }}$ & is & $H$ & is & $H$ & in & $H$ & in & in & 0 & $\mapsto$ & in & $r$ & u \\
\hline 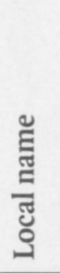 & 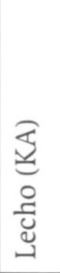 & 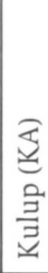 & 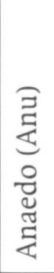 & 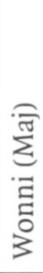 & 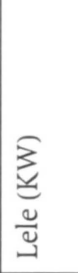 & 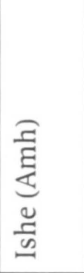 & 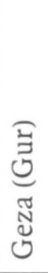 & 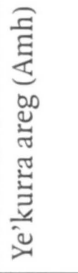 & 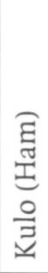 & 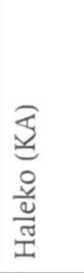 & 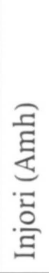 & 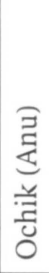 & 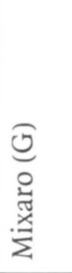 \\
\hline $\begin{array}{l}\text { 裔 } \\
\text { 空 }\end{array}$ & 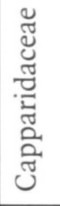 & 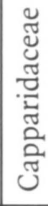 & 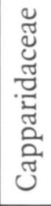 & 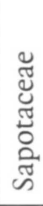 & 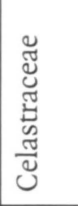 & 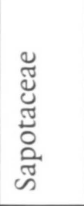 & 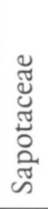 & 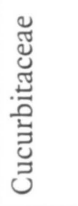 & 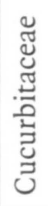 & 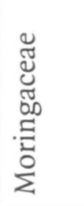 & 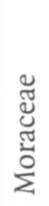 & 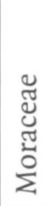 & 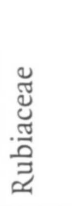 \\
\hline 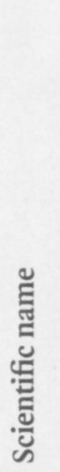 & 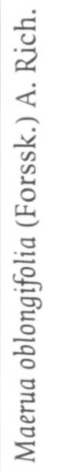 & 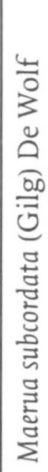 & 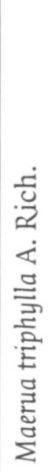 & 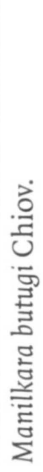 & 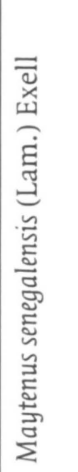 & 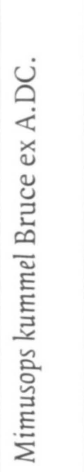 & 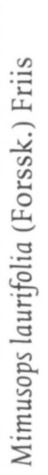 & 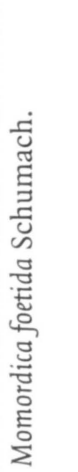 & 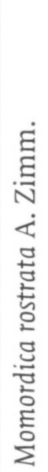 & 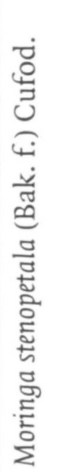 & 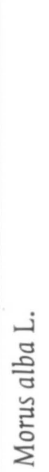 & 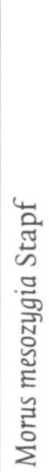 & 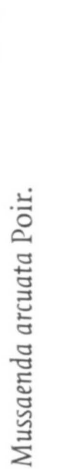 \\
\hline$\stackrel{0}{z}$ & $\stackrel{\sim}{N}^{n}$ & ज़ & బั & స & مे & $\begin{array}{l}\infty \\
0 \\
\text { N }\end{array}$ & ठำ & $\stackrel{\circ}{\stackrel{N}{N}}$ & $\overrightarrow{\hat{N}}$ & $\underset{N}{N}$ & $\stackrel{n}{N}$ & $\frac{4}{4}$ & $\stackrel{n}{\hat{n}}$ \\
\hline
\end{tabular}




\begin{tabular}{|c|c|c|c|c|c|c|c|c|c|c|c|c|c|}
\hline 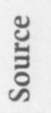 & $\hat{i}$ & $\stackrel{n}{n}$ & $N$ & $\begin{array}{l}n \\
\dot{m} \\
\dot{i}\end{array}$ & $\stackrel{n}{\sim}$ & $m$ & $a$ & $m$ & $\stackrel{n}{\sim}$ & $\begin{array}{l}\dot{0} \\
\dot{m} \\
i=n\end{array}$ & $\hat{i}$ & $\stackrel{n}{\sim}$ & $m$ \\
\hline 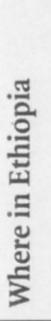 & 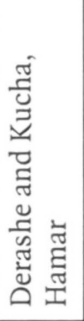 & $\stackrel{\grave{\Xi}}{\Xi}$ & 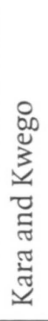 & 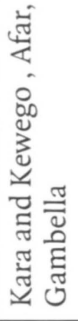 & 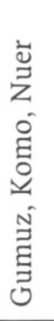 & 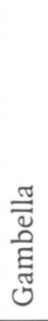 & 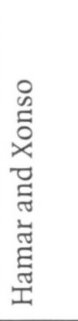 & 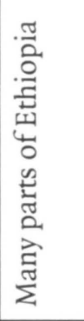 & 营 & 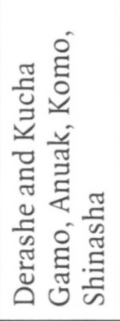 & 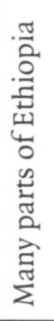 & 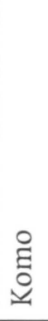 & 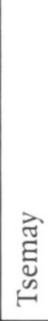 \\
\hline 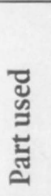 & 茎 & 莺 & $\begin{array}{l}\check{0} \\
\stackrel{2}{2}\end{array}$ & $\begin{array}{l}\breve{\Xi} \\
\stackrel{\Xi}{\Xi}\end{array}$ & 鸪 & 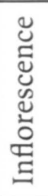 & 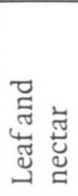 & $\stackrel{\breve{J ~}}{\leftrightarrows}$ & 鸪 & 鸪 & 莺 & 岕 & $\begin{array}{l}2 \\
\vdots \\
0 \\
0 \\
1\end{array}$ \\
\hline $\begin{array}{l}\text { 节 } \\
\text { 吾 }\end{array}$ & 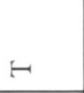 & I & $I$ & I & in & $I$ & I & $H$ & $r$ & is & $I$ & is & is \\
\hline 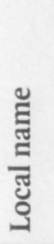 & 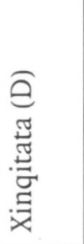 & 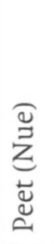 & 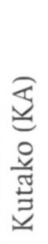 & 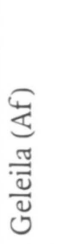 & 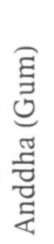 & 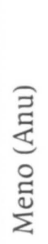 & 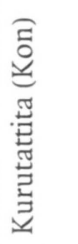 & 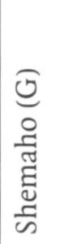 & 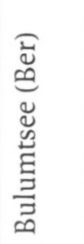 & 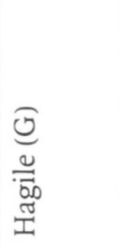 & 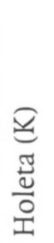 & & 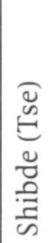 \\
\hline 㙳 & 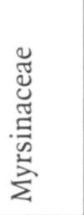 & 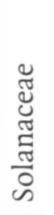 & 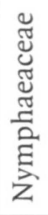 & 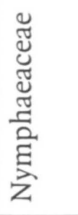 & 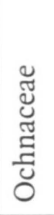 & 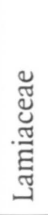 & 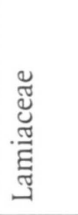 & $\begin{array}{l}\stackrel{\mathscr{J}}{\mathbb{J}} \\
\stackrel{\mathbb{J}}{0}\end{array}$ & 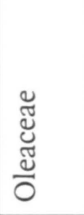 & 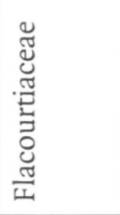 & 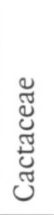 & 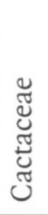 & 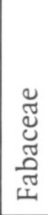 \\
\hline 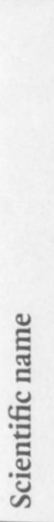 & 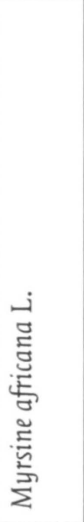 & 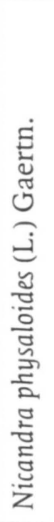 & 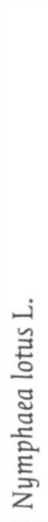 & 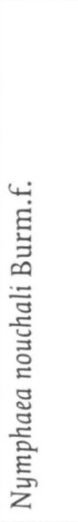 & 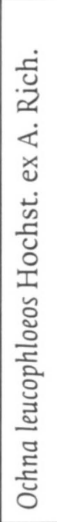 & 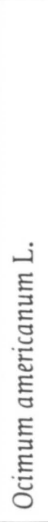 & 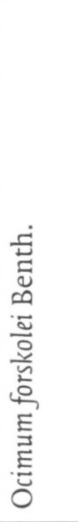 & 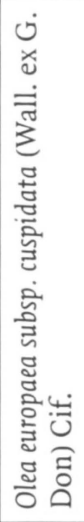 & 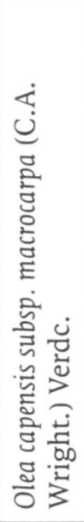 & 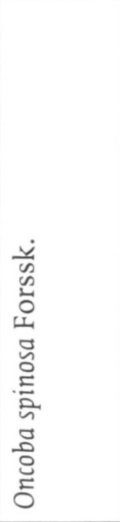 & 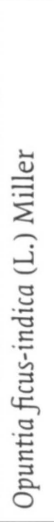 & 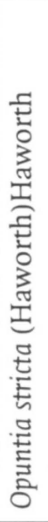 & 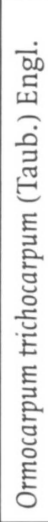 \\
\hline$\grave{z}$ & $\stackrel{\circ}{\circ}$ & $\hat{\widehat{N}}$ & $\stackrel{\infty}{\wedge}$ & શิ & $\infty$ & $\stackrel{\sim}{\infty}$ & $\underset{N}{N}$ & $\underset{N}{\infty}$ & & 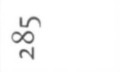 & $\underset{\sim}{\infty}$ & $\underset{N}{\infty}$ & $\underset{N}{\infty}$ \\
\hline
\end{tabular}




\begin{tabular}{|c|c|c|c|c|c|c|c|c|c|c|c|}
\hline 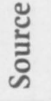 & $\stackrel{n}{\sim}$ & $\tilde{n}$ & $a$ & $\begin{array}{l}0 \\
\text { á }\end{array}$ & $\hat{f}$ & $\stackrel{\circ}{\stackrel{m}{m}}$ & $m$ & $\begin{array}{l}\exists \\
a\end{array}$ & - & $m$ & $a$ \\
\hline 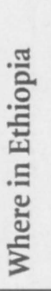 & $\begin{array}{l}\text { 坣 } \\
\text { 宗 }\end{array}$ & 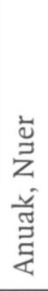 & 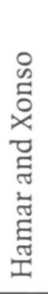 & 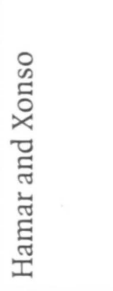 & 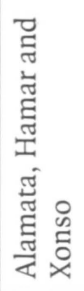 & 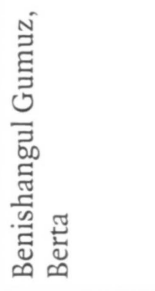 & 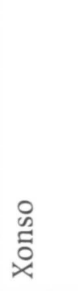 & 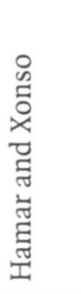 & 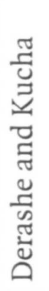 & 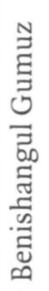 & 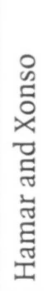 \\
\hline 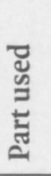 & 芯 & జֶّ & 壱 & 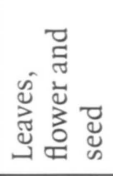 & 荘 & 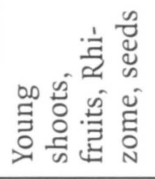 & 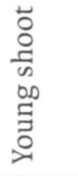 & 苞 & 莺 & 莺 & 鸪 \\
\hline 竞 & I & I & $\infty$ & I & is & $H$ & I & $\mapsto$ & is & - & in \\
\hline 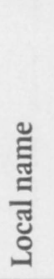 & 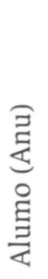 & $\begin{array}{l}\text { 恶 } \\
0 \\
\text { 范 }\end{array}$ & 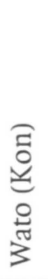 & 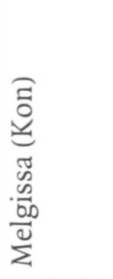 & 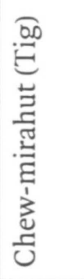 & 莺 & 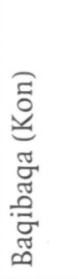 & 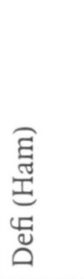 & 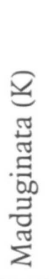 & 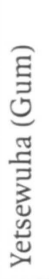 & 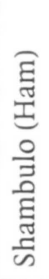 \\
\hline 离 & 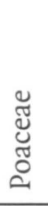 & 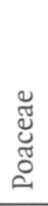 & 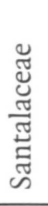 & 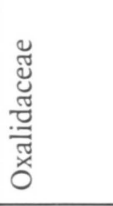 & 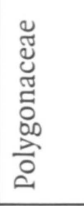 & 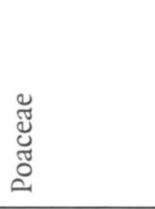 & 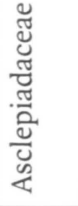 & 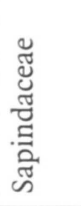 & 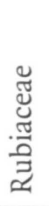 & 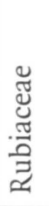 & 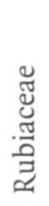 \\
\hline 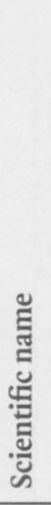 & 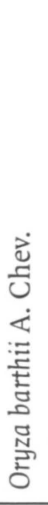 & 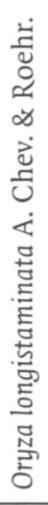 & 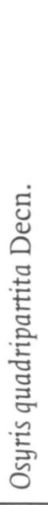 & 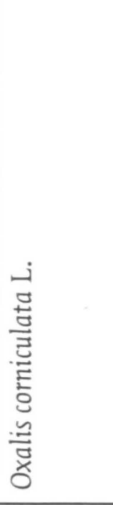 & 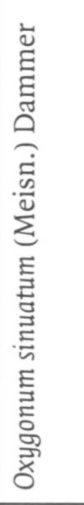 & 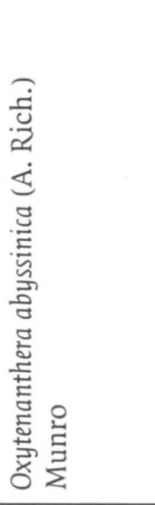 & 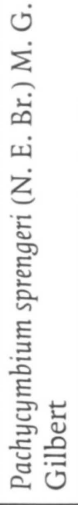 & 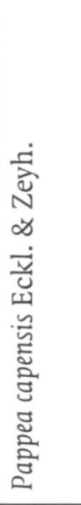 & 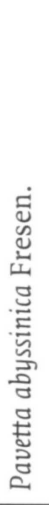 & 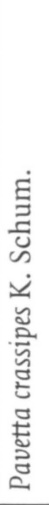 & 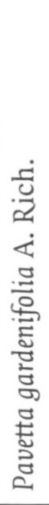 \\
\hline z & ळ્సి & ㅇ. & సे & ๙ิ & $\stackrel{n}{\hat{N}}$ & ন্ড & $\tilde{\text { กิ }}$ & ๙ั & ลิ & ๙্ণ & बे \\
\hline
\end{tabular}




\begin{tabular}{|c|c|c|c|c|c|c|c|c|c|c|c|}
\hline 苟 & - & + & $n$ & $m$ & $\stackrel{n}{=}$ & $\cong$ & $m$ & $\wedge$ & $\stackrel{0}{\text { I }}$ & + & $\begin{array}{l}\stackrel{0}{1} \\
\dot{m} \\
i\end{array}$ \\
\hline 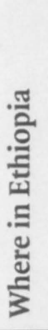 & 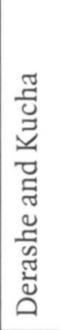 & 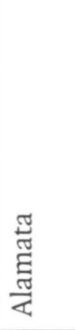 & 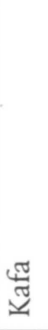 & 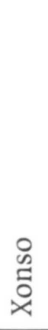 & 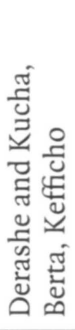 & $\stackrel{\breve{\Xi}}{\Xi}$ & $\stackrel{\vec{n}}{\Xi}$ & $\begin{array}{l}: \overline{\bar{\Xi}} \\
3 \\
3\end{array}$ & 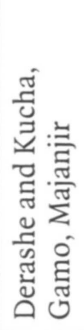 & 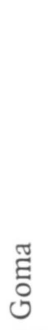 & 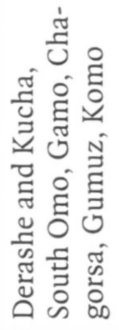 \\
\hline 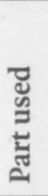 & 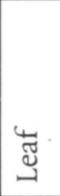 & 莺 & 䔍 & 㟢 & 毂 & 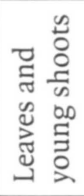 & 叴 & 莺 & 叴 & 亗 & 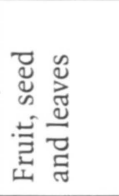 \\
\hline $\begin{array}{l}\text { 节 } \\
\text { 茎 }\end{array}$ & 0 & is & 0 & $u$ & in & $I$ & in & in & $I$ & in & $\mapsto$ \\
\hline 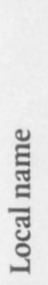 & 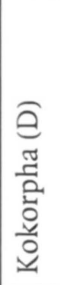 & 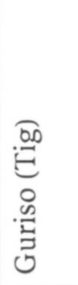 & $\begin{array}{l}\frac{\sigma}{\pi} \\
\stackrel{0}{0} \\
\frac{0}{0}\end{array}$ & 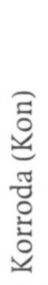 & $\begin{array}{l}\text { อే } \\
\text { है } \\
\text { हु } \\
\text { N }\end{array}$ & 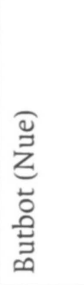 & 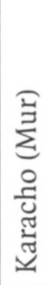 & 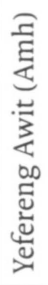 & 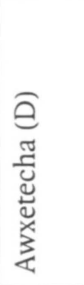 & 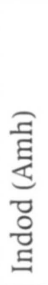 & 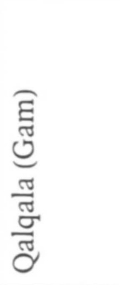 \\
\hline 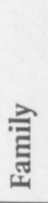 & 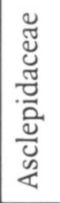 & 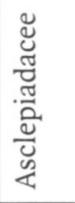 & 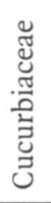 & 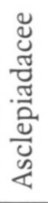 & 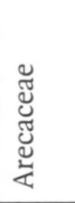 & 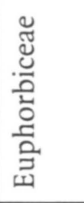 & 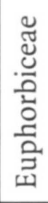 & 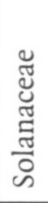 & 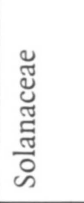 & 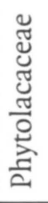 & 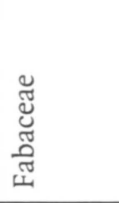 \\
\hline 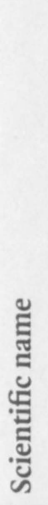 & 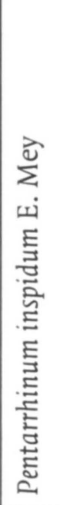 & 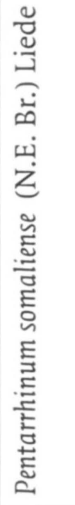 & 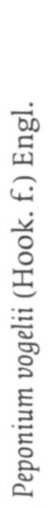 & 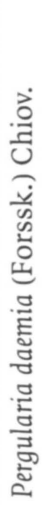 & 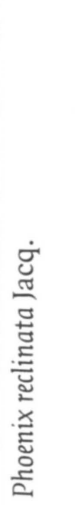 & 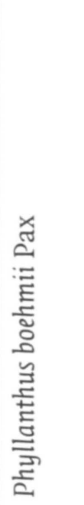 & 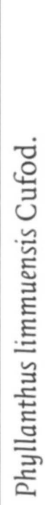 & 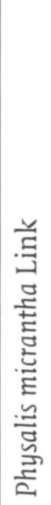 & 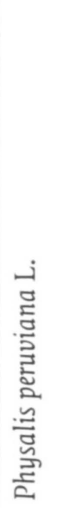 & 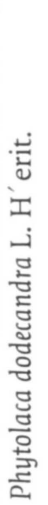 & 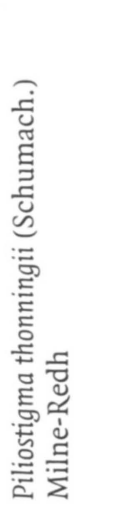 \\
\hline$\stackrel{2}{z}$ & $\stackrel{\circ}{\circ}$ & $\overrightarrow{0}$ & $\stackrel{N}{D}$ & $\stackrel{n}{n}$ & ষ্ণ & $\stackrel{n}{n}$ & : & $\hat{\mathrm{O}}$ & 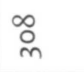 & ò & $\stackrel{\circ}{m}$ \\
\hline
\end{tabular}




\begin{tabular}{|c|c|c|c|c|c|c|c|c|c|c|c|c|}
\hline 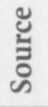 & $m$ & $m$ & $\begin{array}{l}0 \\
\dot{a} \\
\dot{m}\end{array}$ & $\begin{array}{l}a \\
\hat{m} \\
\hat{i}\end{array}$ & $m$ & $a$ & $\bar{\Xi}$ & $m$ & $\hat{m}$ & $a$ & ナ & 음 \\
\hline 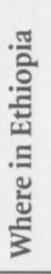 & 莺 & 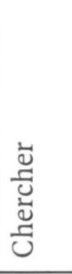 & 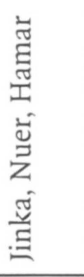 & 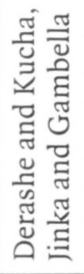 & 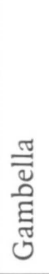 & 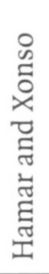 & 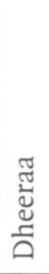 & 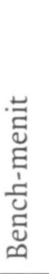 & $\begin{array}{l}0 \\
\stackrel{0}{0} \\
\dot{x}\end{array}$ & 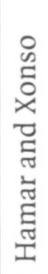 & $\frac{\frac{\pi}{2}}{\frac{\pi}{0}}$ & 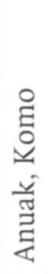 \\
\hline 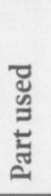 & 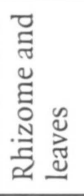 & 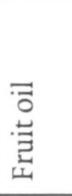 & 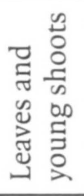 & 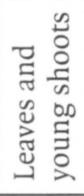 & 隺 & 莺 & 牚 & 鸹 & 鸹 & $\stackrel{\amalg}{\Xi}$ & $\stackrel{\widetilde{J}}{\widetilde{J}}$ & $\begin{array}{l}\stackrel{2}{2} \\
\stackrel{0}{\Xi}\end{array}$ \\
\hline $\begin{array}{l}\text { 节 } \\
\text { 矛 }\end{array}$ & I & $H$ & I & I & $H$ & is & $H$ & 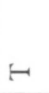 & en & $I$ & is & $u$ \\
\hline 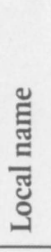 & 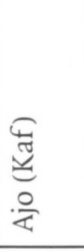 & 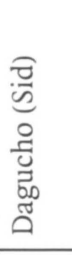 & 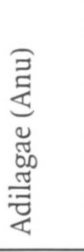 & 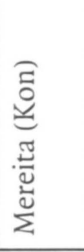 & 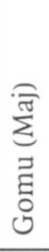 & 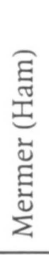 & 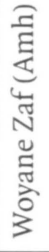 & 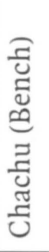 & 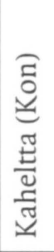 & 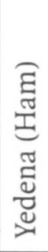 & 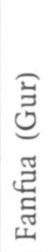 & 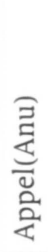 \\
\hline 颃 & 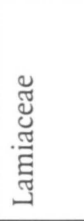 & 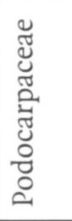 & 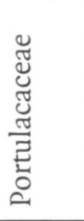 & 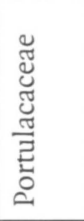 & 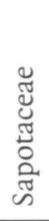 & 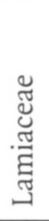 & 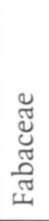 & 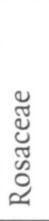 & 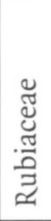 & 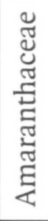 & 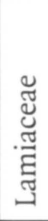 & 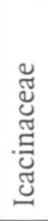 \\
\hline 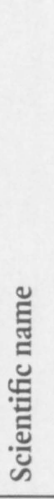 & 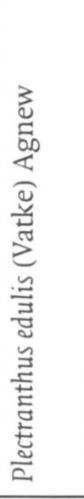 & 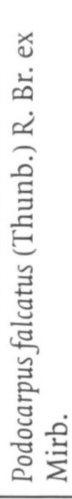 & 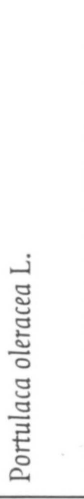 & 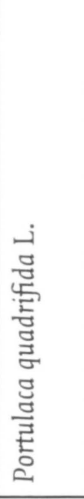 & 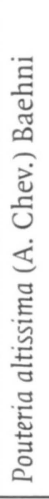 & 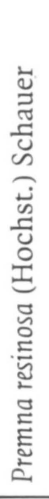 & 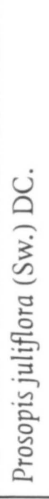 & 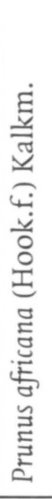 & 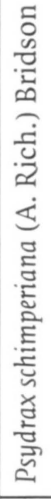 & 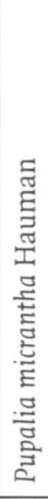 & 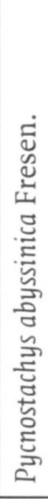 & 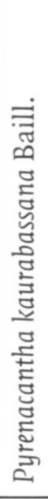 \\
\hline Z & $\vec{m}$ & $\underset{m}{\mathbb{n}}$ & $\stackrel{n}{m}$ & $\underset{m}{\mathbb{N}}$ & $\stackrel{\tilde{m}}{n}$ & $\bar{m}$ & $\vec{m}$ & $\stackrel{\infty}{m}$ & $\stackrel{\vec{m}}{\hat{m}}$ & $\stackrel{\text { I }}{\text { m }}$ & $\vec{N}$ & $\underset{n}{N}$ \\
\hline
\end{tabular}




\begin{tabular}{|c|c|c|c|c|c|c|c|c|c|c|c|c|}
\hline 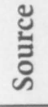 & $\Lambda$ & $\infty$ & $m$ & $m$ & $n$ & $m$ & $\stackrel{n}{i}$ & $n$ & $\Xi$ & $\stackrel{m}{i}$ & $m$ & $\begin{array}{l}\stackrel{\Theta}{m} \\
\dot{m}\end{array}$ \\
\hline 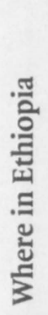 & 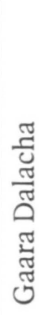 & 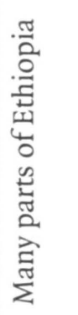 & 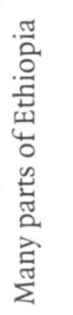 & $\begin{array}{l}\text { 竞 } \\
\text { है } \\
\text { ปึ }\end{array}$ & 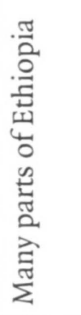 & 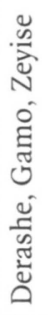 & 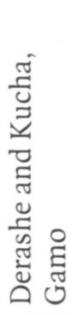 & 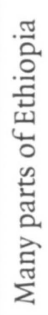 & 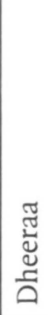 & 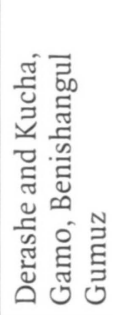 & 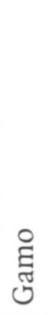 & 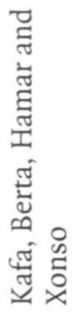 \\
\hline 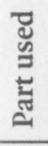 & 鸪 & 卺 & 壱 & 莺 & 壱 & 总 & 芯 & 总 & 莺 & 芯 & 莺 & 莺 \\
\hline $\begin{array}{l}\text { 节 } \\
\text { 吾 }\end{array}$ & in & $r$ & $r$ & 0 & 0 & $\leftarrow$ & in & $\mapsto$ & $\mapsto$ & in & in & in \\
\hline 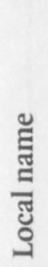 & 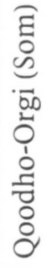 & 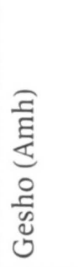 & 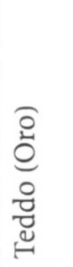 & 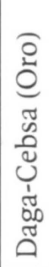 & 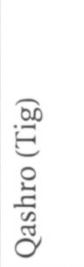 & 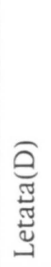 & 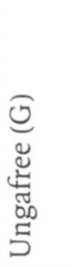 & 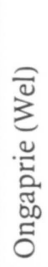 & 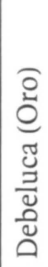 & 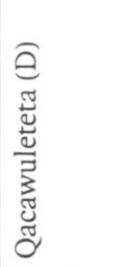 & 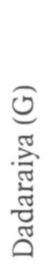 & 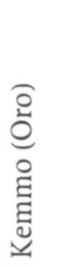 \\
\hline 离 & 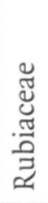 & 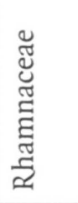 & 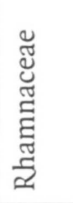 & 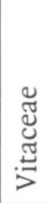 & 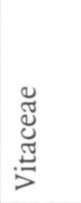 & 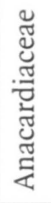 & 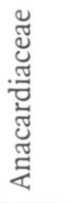 & 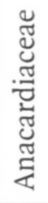 & 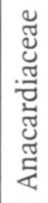 & 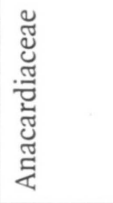 & 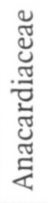 & 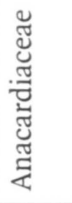 \\
\hline 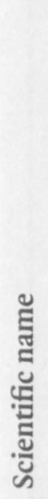 & 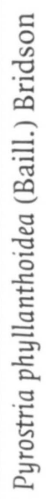 & 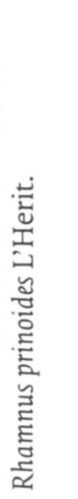 & 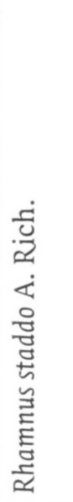 & 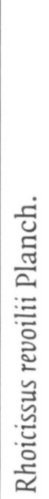 & 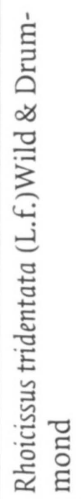 & 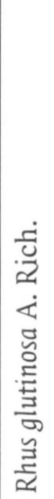 & 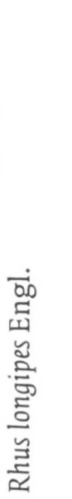 & 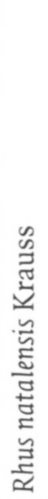 & 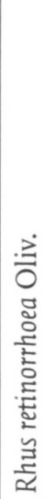 & 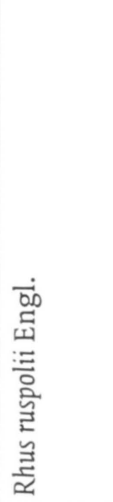 & 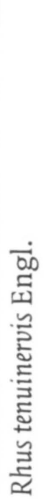 & 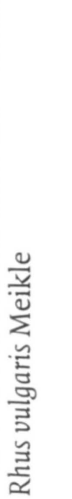 \\
\hline ż & $\underset{\mathfrak{m}}{\mathfrak{m}}$ & $\underset{m}{\mathbb{N}}$ & $\underset{m}{\mathfrak{r}}$ & $\stackrel{\text { D }}{\text { m }}$ & $\underset{m}{\hat{m}}$ & $\underset{m}{\mathbb{m}}$ & $\stackrel{\text { సे }}{m}$ & $\stackrel{\stackrel{m}{m}}{n^{\prime}}$ & $\vec{m}$ & $\stackrel{m}{m}$ & $\stackrel{m}{m}$ & $\stackrel{\dot{m}}{m}$ \\
\hline
\end{tabular}




\begin{tabular}{|c|c|c|c|c|c|c|c|c|c|c|c|}
\hline 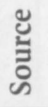 & $a$ & $a$ & $m$ & $m$ & $m$ & $\stackrel{n}{i}$ & $m$ & $m$ & $m$ & $m$ & $m$ \\
\hline 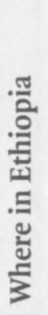 & 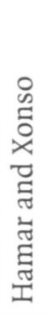 & 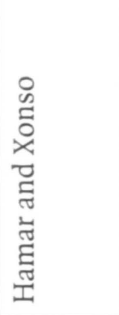 & $\underset{\mathscr{\pi}}{\stackrel{\pi}{\pi}}$ & 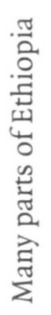 & 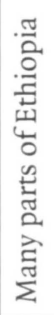 & 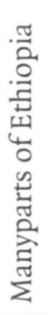 & 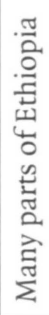 & 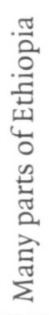 & 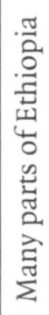 & 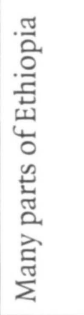 & 交 \\
\hline 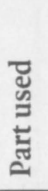 & 总 & 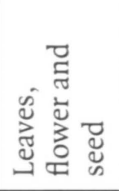 & 壳 & 叴 & 鸪 & 恚 & 总 & 总 & 毠 & 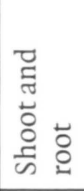 & 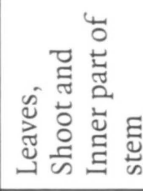 \\
\hline $\begin{array}{l}\frac{\hbar}{5} \\
\text { 荧 }\end{array}$ & es & I & in & is & is & es & $\infty$ & is & is & 工 & is \\
\hline 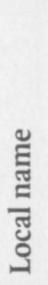 & 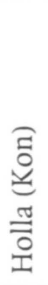 & 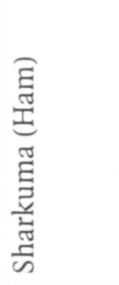 & 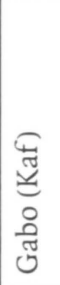 & 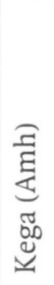 & 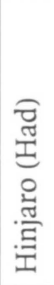 & 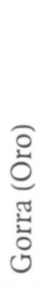 & 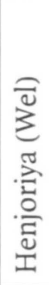 & 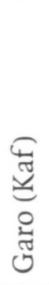 & 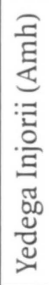 & $\begin{array}{l}\frac{3}{\pi} \\
\frac{\pi}{0} \\
0 \\
0 \\
\frac{0}{0} \\
\frac{0}{0} \\
\frac{1}{n}\end{array}$ & 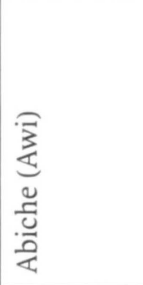 \\
\hline 祘 & $\begin{array}{l}\stackrel{\mathscr{J}}{0} \\
\text { J } \\
\text { त्ञ }\end{array}$ & $\begin{array}{l}\text { चु } \\
\text { पू } \\
\text { त्ञ }\end{array}$ & 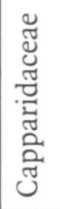 & $\begin{array}{l}\mathscr{J} \\
\stackrel{్}{0} \\
\stackrel{\mathscr{J}}{1}\end{array}$ & 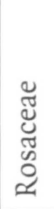 & 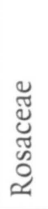 & 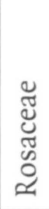 & 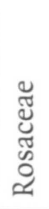 & 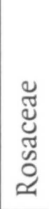 & 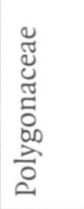 & 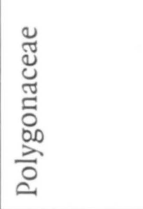 \\
\hline 㫕 & 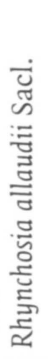 & 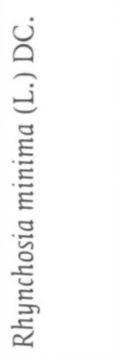 & 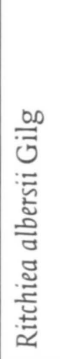 & 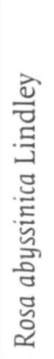 & 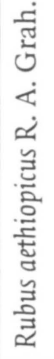 & 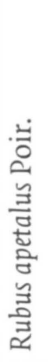 & 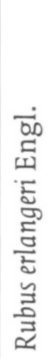 & 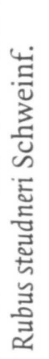 & 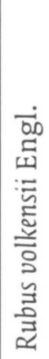 & 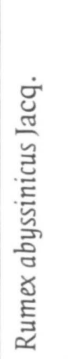 & 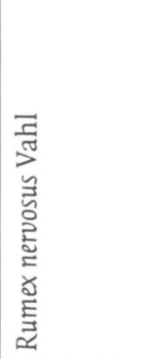 \\
\hline ż & $\stackrel{n}{m}$ & $\stackrel{p}{m}$ & $\hat{m}$ & $\stackrel{m}{m}$ & m & 守 & m & $\stackrel{+}{m}$ & $\stackrel{n}{m}$ & $\underset{m}{J}$ & $\stackrel{\text { q }}{m}$ \\
\hline
\end{tabular}




\begin{tabular}{|c|c|c|c|c|c|c|c|c|c|c|c|}
\hline $\begin{array}{l}\text { : } \\
\text { ¿ू } \\
\text { ¿ }\end{array}$ & + & $\begin{array}{l}n \\
i\end{array}$ & - & 으 & + & $N$ & $m$ & + & + & $a$ & $\stackrel{0}{\stackrel{0}{-1}}$ \\
\hline 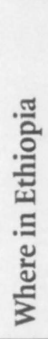 & 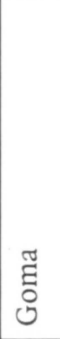 & 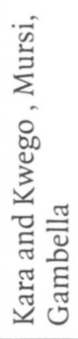 & 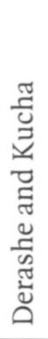 & $\begin{array}{l}\stackrel{\Xi}{0} \\
\cong\end{array}$ & 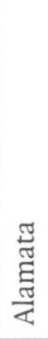 & 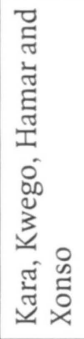 & 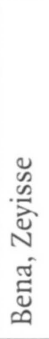 & $\begin{array}{l}\frac{\pi}{2} \\
\frac{\Omega}{0}\end{array}$ & 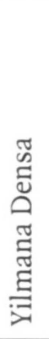 & 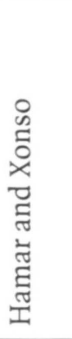 & 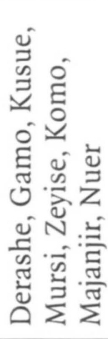 \\
\hline 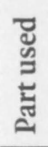 & 莺 & 莺 & E్ & 壭 & 莺 & 鸪 & 㟢 & 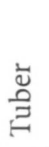 & 壳 & $\begin{array}{l}\breve{\circ} \\
\stackrel{2}{\alpha}\end{array}$ & 蔦 \\
\hline $\begin{array}{l}\text { 总 } \\
\text { 茎 }\end{array}$ & $H$ & $H$ & I & is & is & is & es & I & is & 0 & $H$ \\
\hline 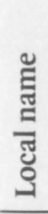 & 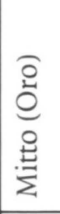 & 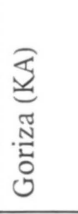 & 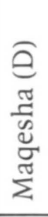 & 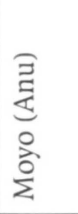 & 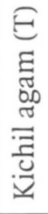 & 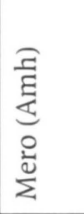 & 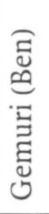 & 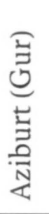 & 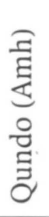 & 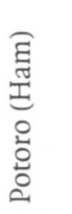 & 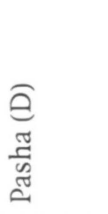 \\
\hline 离 & 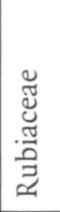 & 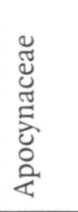 & 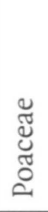 & 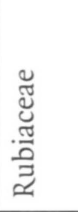 & 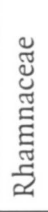 & 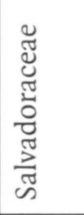 & 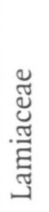 & 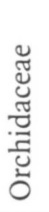 & 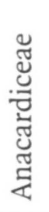 & 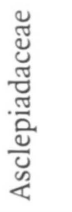 & 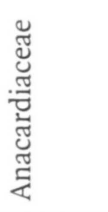 \\
\hline 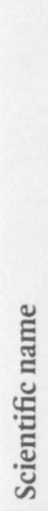 & 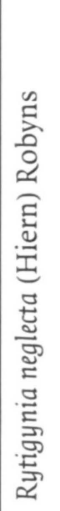 & 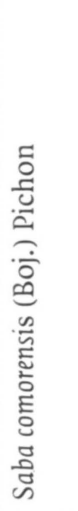 & 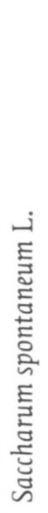 & 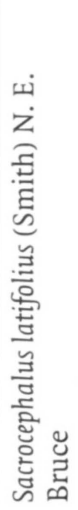 & 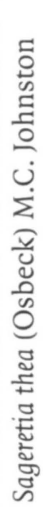 & 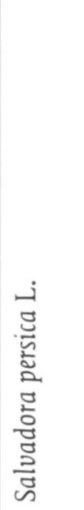 & 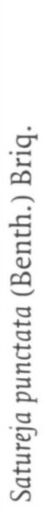 & 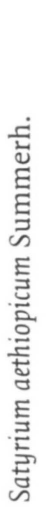 & 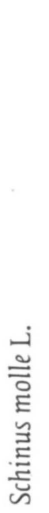 & 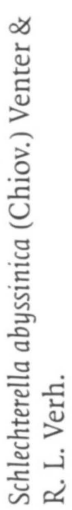 & 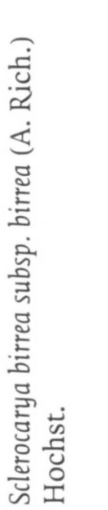 \\
\hline$\stackrel{0}{z}$ & ì & 宇 & $m$ & ga & $\stackrel{m}{m}$ & $\bar{n}$ & $\stackrel{n}{n}$ & $m$ & ñ & $\tilde{n}$ & in \\
\hline
\end{tabular}




\begin{tabular}{|c|c|c|c|c|c|c|c|c|c|c|c|}
\hline $\begin{array}{l}\text { ֻั } \\
\text { ర్ }\end{array}$ & $m$ & $\exists$ & $\begin{array}{l}0 \\
\text { á }\end{array}$ & $m$ & $a$ & 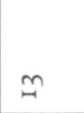 & $m$ & $\wedge$ & $\Lambda$ & 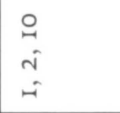 & $\wedge$ \\
\hline 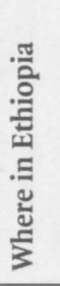 & 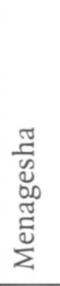 & 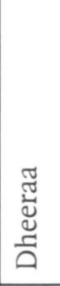 & 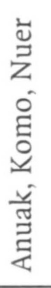 & 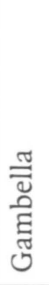 & 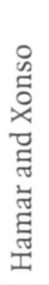 & 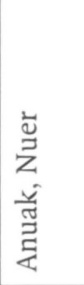 & $\sum_{\Sigma}^{Z}$ & 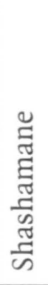 & 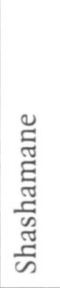 & 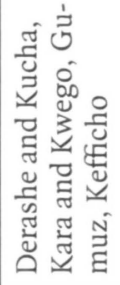 & $\stackrel{\pi}{\bar{\Xi}}$ \\
\hline 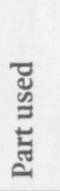 & 㤩 & 壱 & 崩 & 芯 & 芯 & 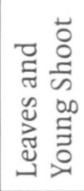 & 莺 & 亗 & 鸹 & 嵌 & 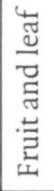 \\
\hline $\begin{array}{l}\text { 节 } \\
\text { 空 }\end{array}$ & $H$ & $\leftarrow$ & is & I & is & 工 & is & is & is & is & is \\
\hline 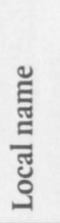 & 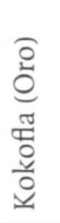 & 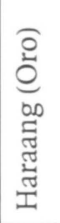 & 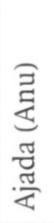 & 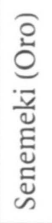 & 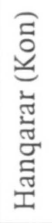 & 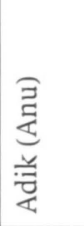 & 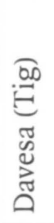 & $\sum_{Z}$ & $\sum_{Z}$ & 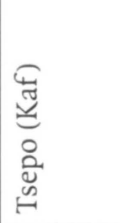 & $\sum_{Z}$ \\
\hline 离 & 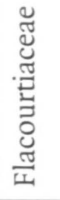 & 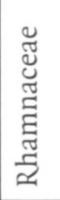 & 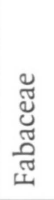 & 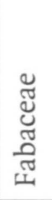 & 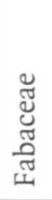 & 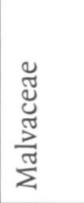 & 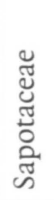 & 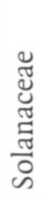 & 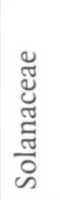 & 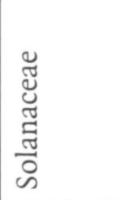 & 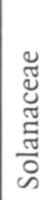 \\
\hline 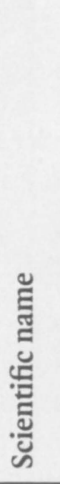 & 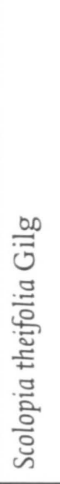 & 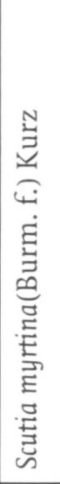 & 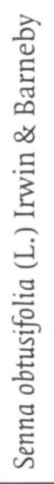 & 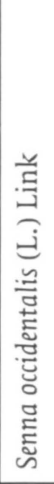 & 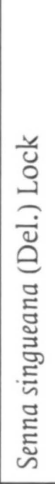 & 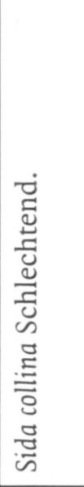 & 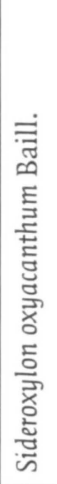 & 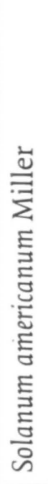 & 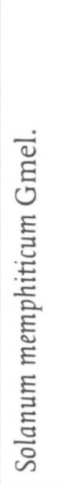 & 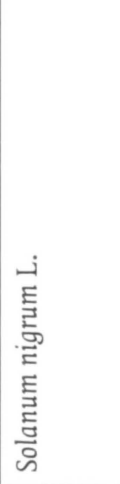 & 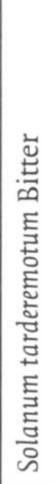 \\
\hline z & 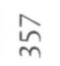 & $\stackrel{\infty}{\stackrel{\infty}{n}}$ & 官 & : & m & 放 & $\stackrel{\tilde{m}}{m}$ & $\stackrel{m}{~}_{n}^{+}$ & $\stackrel{n}{n}_{n}$ & $\stackrel{\circ}{\circ}$ & $\hat{\text { o }}$ \\
\hline
\end{tabular}




\begin{tabular}{|c|c|c|c|c|c|c|c|c|c|c|c|c|}
\hline 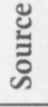 & + & $m$ & + & 0 & $\begin{array}{l}m \\
- \\
-\end{array}$ & $\stackrel{m}{i}$ & $n$ & $\stackrel{n}{=}$ & $m$ & $m$ & $\exists$ & $n$ \\
\hline 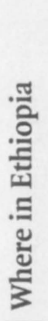 & 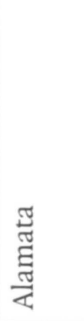 & 豞 & 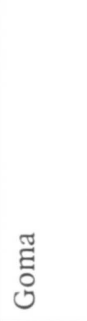 & 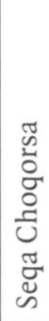 & 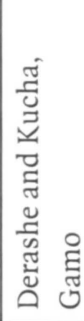 & 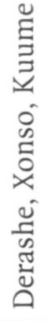 & 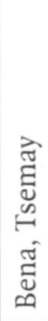 & 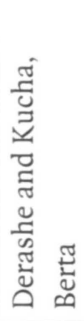 & $\frac{\mathscr{\nu}}{\pi}$ & 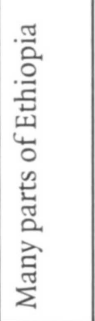 & 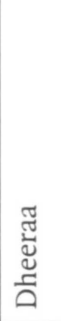 & $\stackrel{\vec{n}}{\stackrel{\overrightarrow{2}}{\Xi}}$ \\
\hline 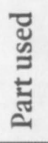 & 䒕 & 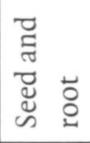 & ¿ूँ & 芯 & $\begin{array}{l}\tilde{\Xi} \\
\tilde{S}\end{array}$ & 芯 & $\begin{array}{l}\vec{U} \\
\tilde{N} \\
\tilde{S}\end{array}$ & 墨 & 鸪 & 茎 & $\stackrel{\breve{J}}{\widetilde{J}}$ & 莽 \\
\hline $\begin{array}{l}\text { 竞 } \\
\text { 空 }\end{array}$ & in & I & I & 工 & I & $H$ & is & in & $H$ & $t$ & I & I \\
\hline 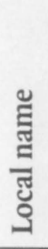 & 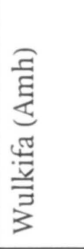 & 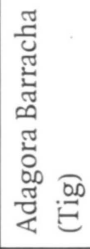 & 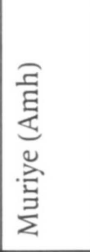 & 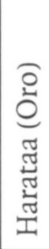 & $\begin{array}{l}0 \\
0 \\
0 \\
0 \\
0 \\
0\end{array}$ & 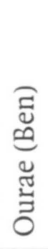 & 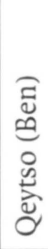 & 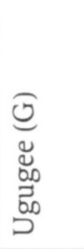 & 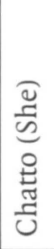 & 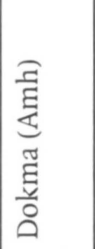 & 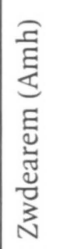 & 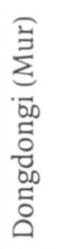 \\
\hline 离 & 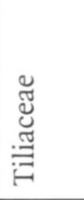 & 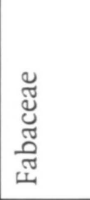 & 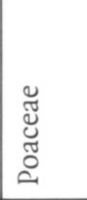 & 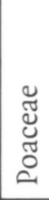 & 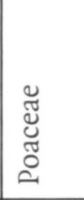 & 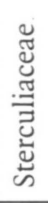 & 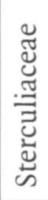 & 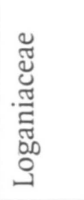 & 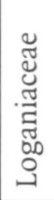 & 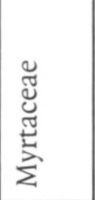 & 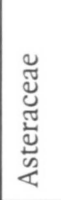 & 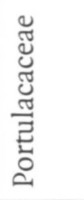 \\
\hline 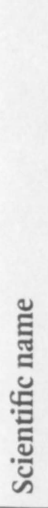 & 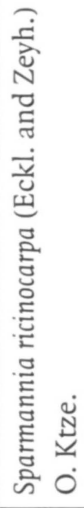 & 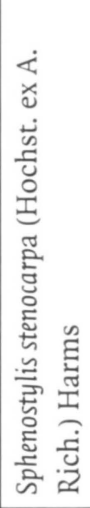 & 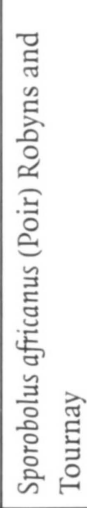 & 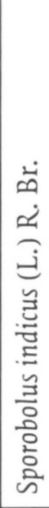 & 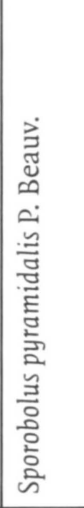 & 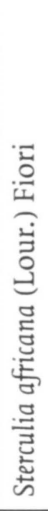 & 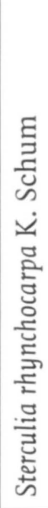 & 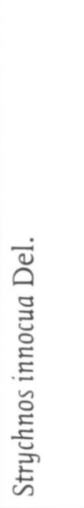 & 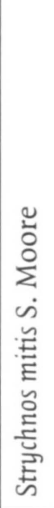 & 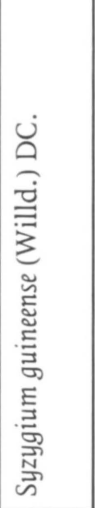 & 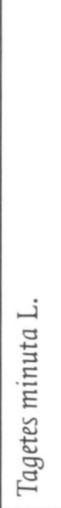 & 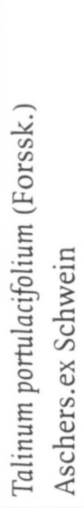 \\
\hline$\stackrel{0}{z}$ & $\begin{array}{l}\infty \\
0 \\
m\end{array}$ & ò & $\stackrel{\circ}{\stackrel{1}{m}}$ & $\vec{m}$ & $\underset{n}{N}$ & $\hat{m}$ & $\frac{j}{m}$ & $\underset{m}{n}$ & $\stackrel{\circ}{m}$ & $\hat{n}$ & $\stackrel{\infty}{\stackrel{\infty}{n}}$ & $\stackrel{a}{m}$ \\
\hline
\end{tabular}




\begin{tabular}{|c|c|c|c|c|c|c|c|c|c|c|c|c|}
\hline $\begin{array}{l}\text { 巳̆ } \\
\text { ¿ू. }\end{array}$ & 엄 & $\begin{array}{l}\circ \\
\text { á }\end{array}$ & $\begin{array}{l}\circ \\
\text { á }\end{array}$ & $n$ & $m$ & $m$ & $m$ & $\begin{array}{l}\stackrel{m}{m} \\
\dot{m}\end{array}$ & $m$ & $m$ & 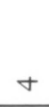 & $\hat{m}$ \\
\hline 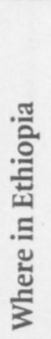 & 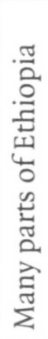 & 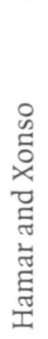 & 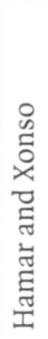 & 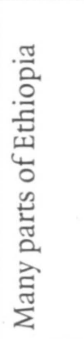 & 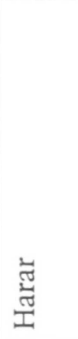 & 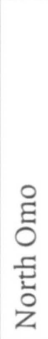 & 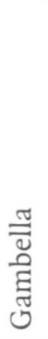 & 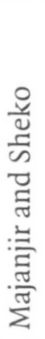 & $\sum_{\Sigma}^{\mathbb{Z}}$ & 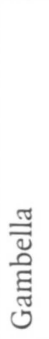 & $\begin{array}{l}\frac{\pi}{0} \\
\frac{J}{0}\end{array}$ & 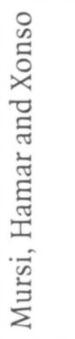 \\
\hline 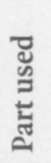 & 㕦 & 莺 & 壱 & 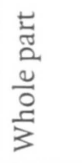 & 莺 & 亗 & 芯 & 壱 & 莺 & 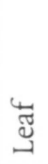 & 鸪 & 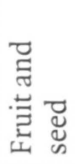 \\
\hline 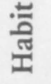 & $\leftarrow$ & in & $r$ & $I$ & in & $I$ & $\leftarrow$ & - & in & I & I & $n$ \\
\hline 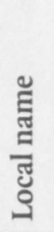 & $\begin{array}{l}\text { (0) } \\
\stackrel{0}{0} \\
0\end{array}$ & 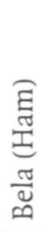 & 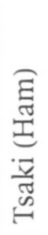 & 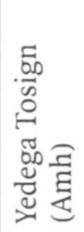 & 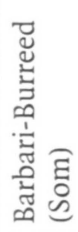 & 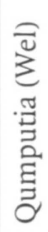 & 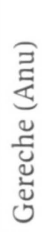 & 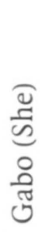 & 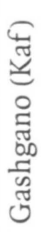 & 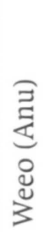 & $\sum_{Z}$ & 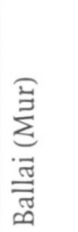 \\
\hline 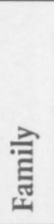 & 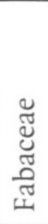 & 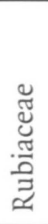 & 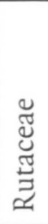 & 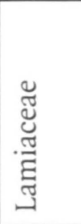 & 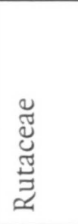 & 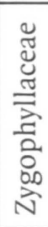 & 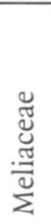 & 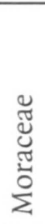 & 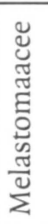 & 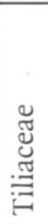 & 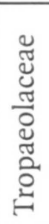 & $\begin{array}{l}\mathscr{J} \\
\stackrel{\mathscr{J}}{~} \\
\text { त्ञ }\end{array}$ \\
\hline 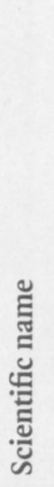 & 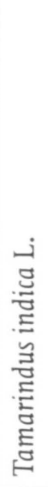 & 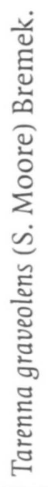 & 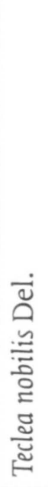 & 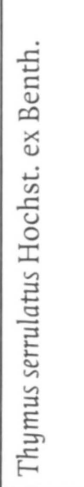 & 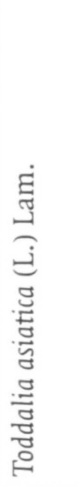 & 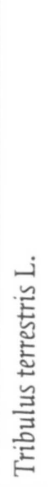 & 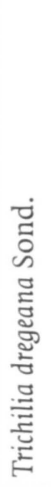 & 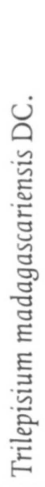 & 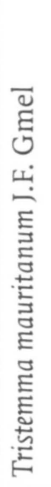 & 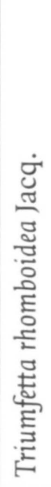 & 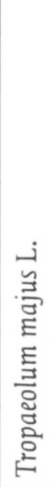 & 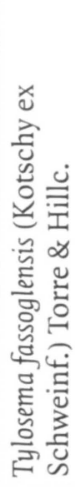 \\
\hline$\grave{z}$ & $\stackrel{\infty}{\infty}$ & $\stackrel{\vec{\infty}}{m}$ & 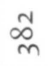 & $\stackrel{m}{\infty}$ & $\stackrel{\infty}{\text { D }}$ & $\stackrel{n}{\infty}$ & $\stackrel{\infty}{m}$ & $\stackrel{\infty}{m}$ & $\stackrel{\infty}{m}$ & ळ) & $\stackrel{\circ}{m}$ & స్ \\
\hline
\end{tabular}




\begin{tabular}{|c|c|c|c|c|c|c|c|c|c|c|c|c|}
\hline 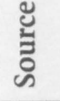 & $m$ & $m$ & $N$ & $\stackrel{m}{\approx}$ & $a$ & $\hat{a}$ & $a$ & $a$ & $\stackrel{m}{n}$ & 0 & $m$ & $m$ \\
\hline 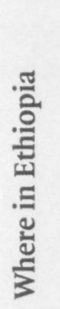 & 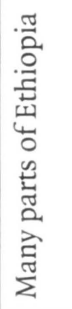 & 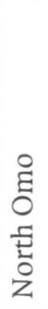 & 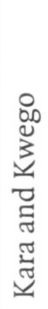 & 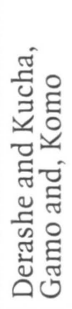 & 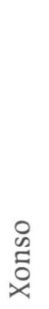 & $\begin{array}{l}0 \\
0 \\
0 \\
\end{array}$ & 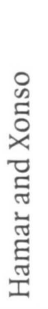 & 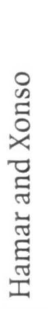 & 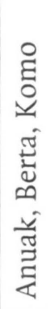 & 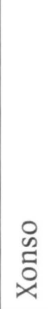 & 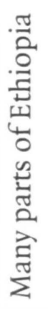 & 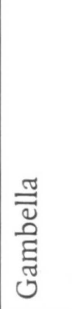 \\
\hline 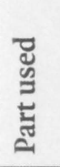 & 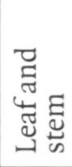 & 壹 & 穿 & 叴 & 莺 & 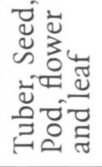 & 䔍 & 莺 & 㟢 & 岂 & 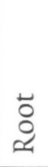 & 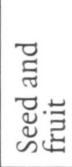 \\
\hline 䓂 & $I$ & in & $\mapsto$ & in & in & 0 & $\infty$ & 0 & $I$ & I & $I$ & $H$ \\
\hline 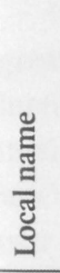 & 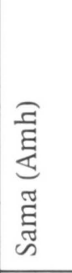 & 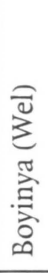 & 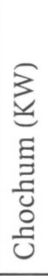 & 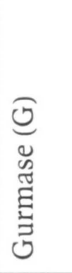 & 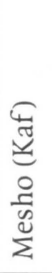 & 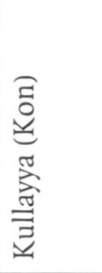 & 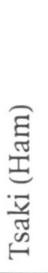 & 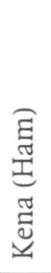 & 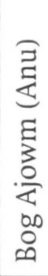 & 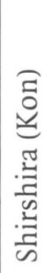 & 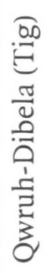 & 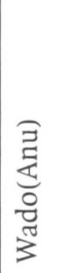 \\
\hline 䘚 & 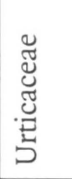 & 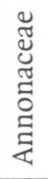 & 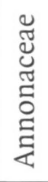 & 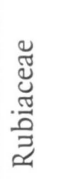 & 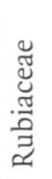 & 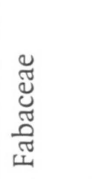 & 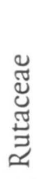 & 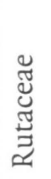 & 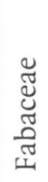 & 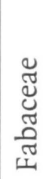 & 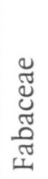 & 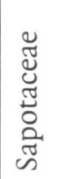 \\
\hline 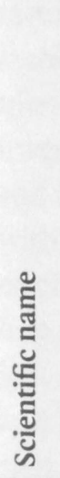 & 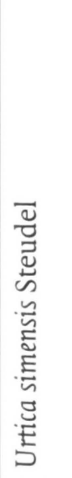 & 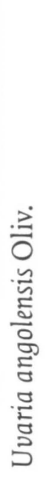 & 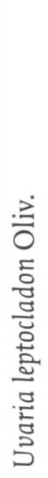 & 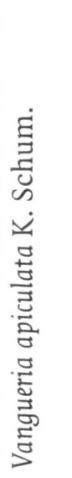 & 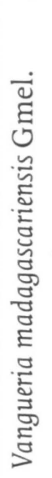 & 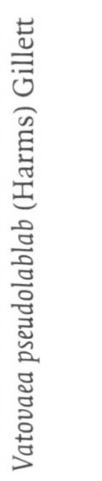 & 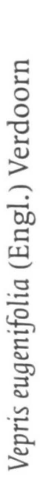 & 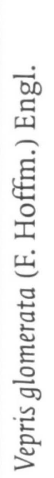 & 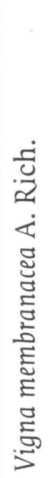 & 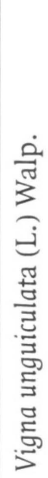 & 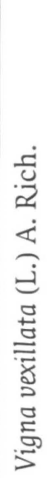 & 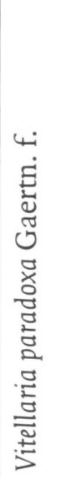 \\
\hline z & ڤે & $\stackrel{n}{m}$ & $\stackrel{+}{m}$ & $\stackrel{\tilde{m}}{m}$ & बे & $\hat{m}$ & 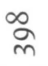 & शे & ঃ & $\vec{o}$ & 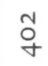 & $\stackrel{n}{q}$ \\
\hline
\end{tabular}




\begin{tabular}{|c|c|c|c|c|c|c|c|c|c|c|}
\hline 苞 & $\begin{array}{l}\stackrel{O}{0} \\
\dot{m}\end{array}$ & $\stackrel{m}{\sim}$ & $\begin{array}{l}O \\
\dot{m}\end{array}$ & $\begin{array}{l}\stackrel{O}{n} \\
\dot{m}\end{array}$ & - & $\stackrel{m}{n}$ & $\stackrel{m}{i}$ & $\stackrel{m}{i}$ & $\begin{array}{l}\hat{a} \\
\hat{n} \\
\hat{i}\end{array}$ & $\begin{array}{l}\hat{a} \\
\hat{n} \\
i=n\end{array}$ \\
\hline 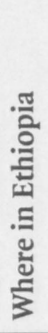 & 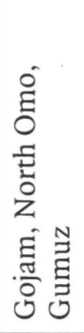 & 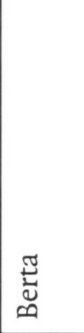 & 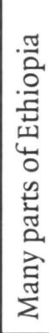 & 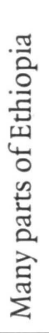 & 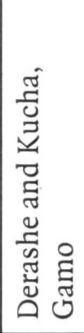 & 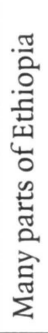 & 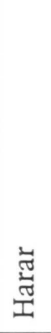 & 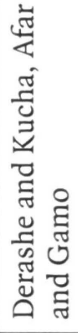 & 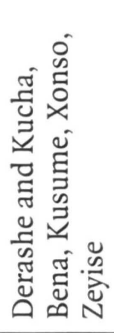 & 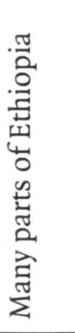 \\
\hline 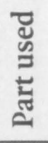 & 壱 & 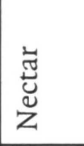 & 鸪 & 壱 & 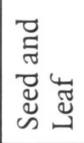 & 鸹 & 总 & 鸪 & 莺 & 莺 \\
\hline $\begin{array}{l}\text { 总 } \\
\text { 吾 }\end{array}$ & 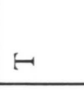 & $\infty$ & 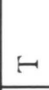 & - & $H$ & is & es & 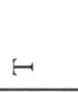 & 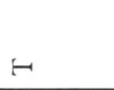 & $r$ \\
\hline 铓 & 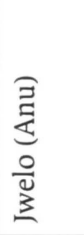 & 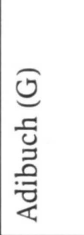 & 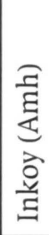 & 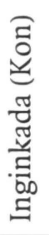 & 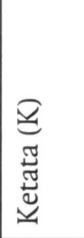 & 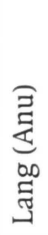 & 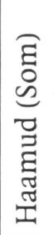 & 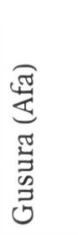 & 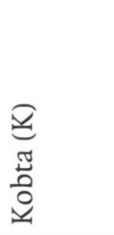 & $\begin{array}{l}\text { ঐ} \\
ٍ \\
z \\
z \\
0 \\
0\end{array}$ \\
\hline 离 & 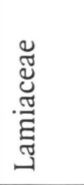 & 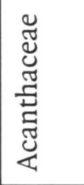 & 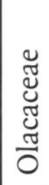 & 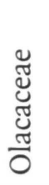 & 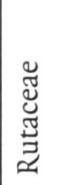 & 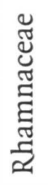 & 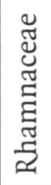 & 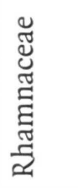 & 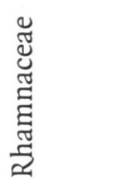 & 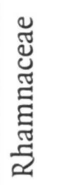 \\
\hline 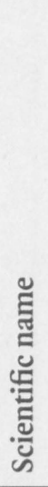 & 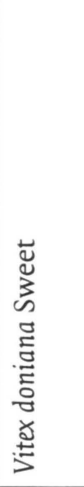 & 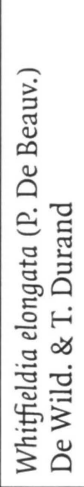 & 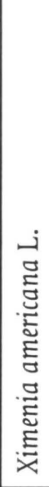 & 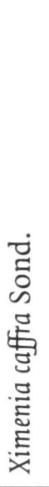 & 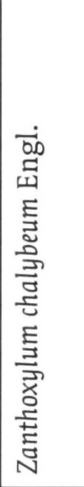 & 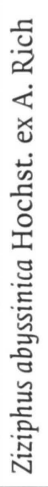 & 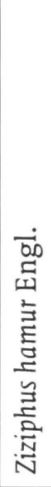 & 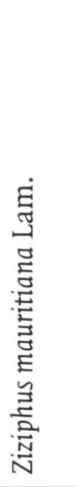 & 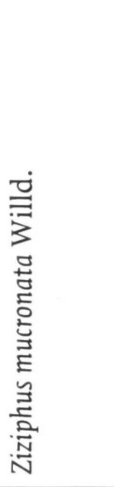 & 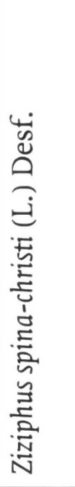 \\
\hline$\stackrel{0}{z}$ & 宫 & 茡 & ঃ & $\hat{o}$ & $\begin{array}{l}\infty \\
\stackrel{+}{+}\end{array}$ & gे & $\stackrel{\circ}{ }$ & F & $\underset{7}{\forall}$ & $\stackrel{n}{F}$ \\
\hline
\end{tabular}

